\title{
A GENERAL THEORY OF RELATIVE REGULAR NEIGHBORHOODS
}

\begin{abstract}
BY
MARSHALL M. COHEN( $\left.{ }^{1}\right)$

Regular neighborhoods of polyhedra in manifolds have been discussed in many papers, most notably, in the present context, in [W], [H-Z], [H] and [S]. We study regular neighborhoods of $X \bmod Y$ in $Z$ where $(Z, X, Y)$ is an arbitrary triad of finite dimensional, locally compact polyhedra. When applied to manifolds our theory generalizes previous results in that we make no compactness assumptions and place no restrictions on the polyhedra $X$ and $Y$ to be considered. The paper is organized as follows:

1. Definitions and notation

2. Simplicial preliminaries

3. The uniqueness theorem

4. The characterization of collared subpolyhedra

5. Cone-retracting and collaring regular neighborhoods

6. The stellar neighborhood theorem

7. Relationships between regular neighborhoods

8. Collapsibility and collapsible retractions

9. Regular neighborhoods via collapsibility.

$V$ is defined to be a regular neighborhood of $X \bmod Y$ in $Z$ if there exists a full triangulation $(J, K, L ; h)$ of $(Z, X, Y)$ in which $V$ underlies a relative first derived neighborhood, $V=h N\left(K-L, J^{\prime}\right)$. Thus, on the one hand, a given regular neighborhood can be presented in an explicit manner with a fixed triangulation. On the other hand, regular neighborhoods are piecewise linear rather than simplicial objects ("there exists a triangulation") and there are uncountably many regular neighborhoods of $X$ mod $Y$ in $Z$ implicit in the choice of triangulating complex and triangulating homeomorphism. We develop the theory by first exploiting the seeming concreteness ( $\$ 2-5)$ and then developing the implicit generality ( $\$ 6-9)$.

After presenting the basic simplicial data we turn immediately to the uniqueness theorem (3.1). (Existence is automatic.) This asserts that, given two regular neighborhoods of $X$ mod $Y$ in $Z$, there is an ambient piecewise linear isotopy taking one onto the other and keeping $X \cup Y$ fixed. The proof is quite direct and carries a great deal of extra information. For example (3.4) if $Z$ is an $n$-manifold the isotopy can be realized by a sequence of $2 n$ "moves relative to $X \cup Y$."
\end{abstract}

Received by the editors July 19, 1967.

(1) Supported by NSF Grant GP-5804 and a D.S.I.R. grant at the 1966 Warwick Topology Symposium. 
The boundary of a regular neighborhood has a two-sided collar which "pinches off" near $Y$ (5.3 and 7.5). The material necessary to prove and to utilize this fact is developed in $\$ 4$. A subsidiary result of this section, irrelevant to the rest of the paper, is the "join-cobordism theorem for polyhedra" (4.3).

The usefulness of a regular neighborhood theory depends on the extent to which it solves the

Recognition Problem. To be able to recognize when a given polyhedron in $Z$ is a regular neighborhood, or is suitably homeomorphic to a regular neighborhood, of $X \bmod Y$ in $Z$.

For, once $V$ and $W$ are known to be homeomorphic to regular neighborhoods, or to be regular neighborhoods, the uniqueness theorem can be invoked to conclude that they are homeomorphic to each other, or to carry one onto the other by an ambient isotopy.

The heart of this paper is the stellar neighborhood theorem (6.1). This says, roughly, that any reasonable candidate for a regular neighborhood (or a regular neighborhood homeomorph) is such if it can be triangulated as a relative stellar neighborhood in its own right-not necessarily as a relative stellar neighborhood in an ambient first derived triangulation, or even as a relative stellar neighborhood in any ambient triangulation. This gives a recognition criterion which is much less stringent than the definition. It makes the theory viable and allows us to answer in $\$ 7$ many of the practical questions which occur when one wishes to use regular neighborhood theory.

It is useful to notice that, through $\S 7$, no mention of collapsing occurs. Thus even when working in manifolds the recognition problem can often be most simply resolved without a collapsing argument.

Finally we turn to collapsibility as a recognition criterion. When the ambient space is a manifold this has been the usual criterion (indeed the definition) because of the correspondence between simplicial collapsing and "shelling" in manifolds. We give a solution to the recognition problem in terms of collapsible retractions (9.1). The existence of a collapsible retraction-i.e. a p.l. retraction with compact, collapsible point inverses-is equivalent ( $(8)$ to the existence of a collapse, but it seems to be a more natural notion in the noncompact case. The retraction takes place "all at once" and one does not have to worry about the order in which things collapse.

This paper assumes Whitehead's results (in (9.2)). Otherwise it is formally independent of and takes a different viewpoint than other developments. However we were strongly motivated by the Hudson-Zeeman paper and by Zeeman's elegant proof $[\mathrm{Z}]$ of the uniqueness of compact, absolute first derived neighborhoods.

\section{Definitions and notation.}

Simplicial conventions. Small Greek letters represent (closed) simplexes. We write $\sigma<J$ if $\sigma$ is a simplex of the complex $J$, and $\sigma<(J-K)$ if $\sigma$ is a simplex of 
$J$ but not of $K$. Lk $(\sigma, J)$ and St $(\sigma, J)=\sigma \operatorname{Lk}(\sigma, J)$ denote the link and the star of $\sigma$ in $J$.

By a complex we mean a locally finite simplicial complex in some Euclidean space $R^{q}$. The letters $J, K, L$ always represent complexes. The assertion $K<J$ means that $K$ is a subcomplex of $J$, and the assertion $K \triangleleft J$ means that $K$ is a full subcomplex of $J$ (i.e. $\sigma \cap K$ is a simplex for each $\sigma<J$ ). We write $K \cong J$ to indicate that $K$ and $J$ are simplicially isomorphic. $|K|$ denotes the space underlying $K$.

If $S$ is a set (which may or may not be contained in $|J|$ ) then we denote

$$
\begin{aligned}
& N(S, J)=\{\sigma<J \mid \sigma \text { is the face of a simplex of } J \text { which meets } S\}, \\
& C(S, J)=\{\sigma<J \mid \sigma \cap S=\varnothing\}, \\
& \dot{N}(S, J)=N(S, J) \cap C(S, J) .
\end{aligned}
$$

We often write $N\left(K-L, J_{*}\right)$ instead of $N\left(|K|-|L|, J_{*}\right)$, when $K, L<J$ and $J_{*}$ is a partition of $J$. In general, we will be quick to write $K$ instead of $|K|$ and $K-L$ instead of $|K|-|L|$, if no confusion can occur.

Two simplexes $\sigma=\sigma^{i}$ and $\tau=\tau^{j}$ are joinable if their vertices span an $(i+j+1)$ simplex, $\sigma \tau$. Two complexes $K$ and $J$ in $R^{q}$ are joinable if $\sigma$ and $\tau$ are joinable for each $\sigma<K, \tau<J$ and if $(\sigma \tau) \cap\left(\sigma_{1} \tau_{1}\right)=\left(\sigma \cap \sigma_{1}\right)\left(\tau \cap \tau_{1}\right)$ for each $\sigma, \sigma_{1}<K$ and $\tau, \tau_{1}<J$. The empty simplex $\varnothing$ belongs to every complex and $\varnothing \sigma=\sigma$ for every simplex $\sigma$. The join of two joinable complexes is denoted by $K J$ - or for emphasis by $K * J$-and is given by

$$
K J=K * J=\{\sigma \tau \mid \sigma<K, \tau<J\} .
$$

Although $K J$ is not locally finite in general, we will only deal with locally finite subcomplexes of $K J$. If $f: K_{1} \rightarrow K_{2}$ and $g: J_{1} \rightarrow J_{2}$, where $K_{i}$ is joinable with $J_{i}$, then $(f * g): K_{1} J_{1} \rightarrow K_{2} J_{2}$ is defined by the condition that

$$
(f * g)(t x+(1-t) y)=t f(x)+(1-t) g(y)
$$

for each $x \in K_{1}$ and $y \in J_{1}$.

If $\sigma<J$ then $J^{\prime}$ denotes a first derived of $J$ and $b(\sigma)$ denotes the distinguished interior point of $\sigma$. (It is often important not to choose $b(\sigma)$ as the actual barycenter.) If we write $A=b\left(\sigma_{0}\right) \cdots b\left(\sigma_{q}\right)<J^{\prime}$ it is understood that $\sigma_{0}<\sigma_{1}<\cdots<\sigma_{q}$. If $\sigma<J$ then the dual to $\sigma$ in $J, D(\sigma, J)$, and its subcomplex $\dot{D}(\sigma, J)$ are defined by

and

$$
D(\sigma, J)=\left\{b\left(\sigma_{0}\right) \cdots b\left(\sigma_{q}\right) \mid \sigma<\sigma_{0}<\cdots<\sigma_{q}<J\right\}<J^{\prime}
$$

$$
\dot{D}(\sigma, J)=\left\{b\left(\sigma_{0}\right) \cdots b\left(\sigma_{q}\right) \mid \sigma \supsetneqq \sigma_{0}<\cdots<\sigma_{q}<J\right\}<J^{\prime} .
$$

We shall repeatedly use the fact that $\dot{D}(\sigma, J) \cong \mathrm{Lk}(\sigma, J)^{\prime}$ under the isomorphism taking $b\left(\sigma \alpha_{0}\right) b\left(\sigma \alpha_{1}\right) \cdots b\left(\sigma \alpha_{q}\right)$ onto $b\left(\alpha_{0}\right) b\left(\alpha_{1}\right) \cdots b\left(\alpha_{q}\right)$. More generally, if $f: J \rightarrow K$ is a simplicial mapping, if $f: J^{\prime} \rightarrow K^{\prime}$ is also simplicial, and if $\sigma<K$ then

$$
\begin{aligned}
D(\sigma, f) & \equiv f^{-1} D(\sigma, K) \\
& =\left\{b\left(\tau_{0}\right) \cdots b\left(\tau_{q}\right) \mid \sigma<f\left(\tau_{0}\right), \tau_{0}<\cdots<\tau_{q}<J\right\}<J^{\prime} .
\end{aligned}
$$

$\dot{D}(\sigma, f)$ is defined correspondingly. These duals are studied in detail in $\S 5$ of [C]. 
Polyhedral concentions. A triangulation of a topological space $T$ is a pair $(J, h)$ where $J$ is a simplicial complex and $h: J \rightarrow T$ is a homeomorphism. A polyhedron consists of a space $X$ and a maximal family of piecewise-linearly related triangulations of $X$. When speaking of a triangulation of a polyhedron we always mean a triangulation in the given family. If $Z$ and $X$ are polyhedra, with $X$ contained in $Z$, then $X$ is a subpolyhedron of $Z(X<Z)$ if there is a triangulation $(J, h)$ of $Z$ and a subcomplex $K$ of $J$ such that $(K, h \mid K)$ is a triangulation of $X$. In this case we say that $(J, h)$ is an extension of $(K, h \mid K)$. More generally, if $X<Z, Y<Z$, and $\left\{Z_{i}\right\}$ is a family of subpolyhedra of $Z$ then $\left(J,\left\{J_{i}\right\}, K, L ; h\right)$ is a triangulation of $\left(Z,\left\{Z_{i}\right\}, X, Y\right)$ if the $J_{i}, K$ and $L$ are subcomplexes of $J$ such that $h\left(J_{i}\right)=Z_{i}, h(K)=X$ and $h(L)=Y$. If, further, $K$ and $L$ are full in $J$ then this is called a full triangulation of $\left(Z,\left\{Z_{i}\right\}, X, Y\right)$.

All mappings and manifolds considered are piecewise linear. We write $X \simeq Y$ to indicate that $X$ and $Y$ are piecewise linearly homeomorphic.

$\mathrm{Bdy}_{Z} X$ and $\mathrm{Int}_{z} X$ denote the topological boundary and interior of $X$ in $Z$. The notation $\partial X$ is reserved (unless otherwise stipulated) for the boundary of a manifold. The closure of a set $S$ is denoted by $\mathrm{Cl}(S)$ or by $\bar{S}$. Finally, if $X, Y<Z$ we denote

$$
\mathrm{Cl}(X-Y)=X_{R} ; \quad \mathrm{Cl}(X-Y) \cap Y=Y_{R} .
$$

(The “ $R$ ” stands for “ relativized." Hudson-Zeeman denoted these sets by $X_{\natural}$ and $Y_{\mathrm{\natural}}$.) This notation will only be used with $X$ 's and $Y$ 's, so no confusion should occur.

Regular neighborhoods. If $X, Y, V<Z$ then $V$ is a regular neighborhood of $X \bmod Y$ in $Z$ if there exists a full triangulation $(J, K, L ; h)$ of $(Z, X, Y)$ and a first derived $J^{\prime}$ such that $V=h N\left(K-L, J^{\prime}\right)$. More generally, $\left(V,\left\{V_{i}\right\}\right)$ is a regular neighborhood of $X \bmod Y$ in $\left(Z,\left\{Z_{i}\right\}\right)$ if there is a full triangulation $\left(J,\left\{J_{i}\right\}, K, L ; h\right)$ of $\left(Z,\left\{Z_{i}\right\}, X, Y\right)$ such that $V=h N\left(K-L, J^{\prime}\right)$ and $V_{i}=h N\left(K-L, J_{i}^{\prime}\right) \equiv$ $h N\left(K \cap J_{i}-L \cap J_{i}, J_{i}^{\prime}\right)$.

Notice that, if $g: Z \rightarrow Z$ is a homeomorphism fixed on $X \cup Y$, and if $V$ is a regular neighborhood of $X \bmod Y$ in $Z$ via the triangulation $(J, K, L ; h)$, as above, then $g(V)$ is a regular neighborhood of $X \bmod Y$ in $Z$ via the triangulation $(J, K, L ; g h)$. Thus, though most authors suppress the triangulating homeomorphism, it will often be essential that we keep track of it.

2. Simplicial preliminaries. In this section we present the basic simplicial data which will be used throughout the paper.

Lemma 2.1. If $K \triangleleft J$ and $Q<J$ then $K \cap Q \triangleleft Q$.

LEMMA 2.2. If $L \triangleleft K \triangleleft J$ then every simplex $\sigma<N(K, J)$ is uniquely expressible as $\sigma=\alpha \beta \gamma$ where $\alpha<L, \beta<C(L, K)$ and $\gamma<\dot{N}(K, J)$. Moreover if $\sigma$ is a principal simplex of $N(K, J)$ then $(\alpha \beta) \neq \varnothing$.

Relative stellar neighborhoods. If $K, L<J$ we denote

$$
K_{R}=N(K-L, K) ; \quad L_{R}=L \cap K_{R} .
$$


This notation will only be used for complexes denoted by $K$ 's and $L$ 's so no confusion should arise. Since our complexes have the weak topology this notation is connected with the corresponding notation for polyhedra by

Lemma 2.3. If $X=|K|$ and $Y=|L|$ then $X_{R}=\left|K_{R}\right|$ and $Y_{R}=\left|L_{R}\right|$.

Corollary 2.4. If $K, L<J$ and $J_{*}$ is a partition of $J$ inducing partitions $K_{*}, L_{*}$ then $\left(K_{*}\right)_{R}=\left(K_{R}\right)_{*}$ and $\left(L_{*}\right)_{R}=\left(L_{R}\right)_{*}$.

Lemma 2.5. If $K, L<J$ and $J_{*}$ is a partition of $J$ then

$$
N\left(K-L, J_{*}\right)=\bigcup\left\{N\left(K-L, \sigma_{*}\right) \mid \sigma<J, \sigma \cap(K-L) \neq \varnothing\right\} .
$$

LeMmA 2.6. If $K, L<J_{0} \triangleleft J$ then $N\left(K-L, J_{0}\right)=N(K-L, J) \cap J_{0}$.

Proof. If $\sigma$ is a principal simplex of $N\left(K-L, J_{0}\right)$ then $\sigma$ is a simplex of $J_{0}$ meeting $K-L$. So $\sigma<N(K-L, J) \cap J_{0}$. If on the other hand $\sigma<N(K-L, J) \cap J_{0}$ then $\sigma<J_{0}$ and there exists a simplex $\tau$ such that $\sigma<\tau<J$ and $\tau \cap(K-L) \neq \varnothing$. Then, since $J_{0}$ is full, $\tau \cap J_{0}=\tau_{0}$ is a simplex which clearly contains $\sigma \cup(\tau \cap K)$. Thus $\tau_{0}$ is a simplex of $J_{0}$ such that $\sigma<\tau_{0}$ and $\tau_{0} \cap(K-L) \neq \varnothing$. Hence $\sigma<N\left(K-L, J_{0}\right)$, q.e.d.

Corollary 2.7. If $L<J$ and $K \triangleleft J$ then $K_{R}=K \cap N(K-L, J)$.

Proof. $K \cap L<K \triangleleft J$. Therefore, by (2.6),

$$
K \cap N(K-(L \cap K), J)=N(K-(L \cap K), K)=N(K-L, K)=K_{R} .
$$

COROllary 2.8. If $K, L \triangleleft J$ then

(1) $L_{R} \triangleleft K_{R} \triangleleft N(K-L, J)$,

(2) $N(K-L, J)=N\left(K_{R}-L_{R}, J\right)=N\left(K_{R}, N(K-L, J)\right)$.

Proof. $L \triangleleft J$ and $L_{R}=L \cap K_{R}$. Also $K \triangleleft J$ and $K_{R}=K \cap N(K-L, J)$. Therefore (1) follows from (2.1). Assertion (2) is immediate, q.e.d.

We come now to the basic fact about relative stellar neighborhoods.

Proposition 2.9. If $K, L \triangleleft J$ then every simplex $\sigma$ of $N(K-L, J)$ is uniquely expressible as $\sigma=\alpha \beta \gamma$ where $\alpha<L_{R}, \beta<C\left(L_{R}, K_{R}\right)=C(L, K)$ and

$$
\gamma<\dot{N}\left(K_{R}, N(K-L, J)\right) \text {. }
$$

Further, if $\sigma$ is principal then $\beta \neq \varnothing$.

Proof. This is an immediate consequence of (2.8) and (2.2), except for the last assertion. But from (2.2) we see that if $\sigma$ is principal then $(\alpha \beta) \neq \varnothing$. Since no principal simplex of $K_{R}$ lies in $L_{R}$ this implies that $\beta \neq \varnothing$, q.e.d.

Convention. If $K, L \triangleleft J$ then the assertion that $\alpha \beta \gamma<N(K-L, J)$ carries with it the fact that $\alpha<L_{R}, \beta<C\left(L_{R}, K_{R}\right)$ and $\gamma<\dot{N}\left(K_{R}, N(K-L, J)\right)$.

Proposition 2.9 allows us to make the following

Definition. Let $a=(0,0), b_{0}=(1,0)$ and $b_{1}=(1,1) \in R^{2}$. Let $\Delta=a b_{0} b_{1}$. If $K$ and $L$ are full subcomplexes of $J$ then the natural parameterization of $N(K-L, J)$ is the 
unique simplicial mapping $\eta: N(K-L, J) \rightarrow \Delta$ such that $\eta(\alpha)=a, \eta(\beta)=b_{0}$ and $\eta(\gamma)=b_{1}$, for every simplex $\alpha \beta \gamma<N(K-L, J)$.

Relative first derived neighborhoods. It is clear that $\left|N\left(K-L, J^{\prime}\right)\right|<|N(K-L, J)|$. This, (2.5) and (2.9) yield

LEMma 2.10. If $K, L \triangleleft J$ then the simplexes of $N\left(K-L, J^{\prime}\right)$ are precisely those simplexes of $J^{\prime}$ of the form

$$
A=b\left(\alpha_{0} \beta_{0} \gamma_{0}\right) b\left(\alpha_{1} \beta_{1} \gamma_{1}\right) \cdots b\left(\alpha_{q} \beta_{q} \gamma_{q}\right)
$$

where $\alpha_{i} \beta_{i} \gamma_{i}<N(K-L, J)$ and, for each $i, \gamma_{i}=\varnothing$ or $\beta_{i} \neq \varnothing \neq \gamma_{i}$. The simplexes of $\dot{N}\left(K-L, J^{\prime}\right)$ are precisely those of $N\left(K-L, J^{\prime}\right)$ such that $\beta_{i}=\varnothing=\gamma_{i}$ or $\beta_{i} \neq \varnothing \neq \gamma_{i}$, for each $i$.

Corollary 2.11. If $K, L \triangleleft J$ then $N\left(K-L, J^{\prime}\right)$ and $\dot{N}\left(K-L, J^{\prime}\right)$ are full in $J^{\prime}$. Also, $N\left(K-L, J^{\prime}\right) \cap L^{\prime}=L_{R}^{\prime}$.

LEMMA 2.12. If $K, L \triangleleft J$ then

$$
\left|\dot{N}\left(K-L, J^{\prime}\right)\right|=\left(\mathrm{Bdy}_{|J|}\left|N\left(K-L, J^{\prime}\right)\right|\right) \cup\left|L_{R}^{\prime}\right| .
$$

LEMMA 2.13. If $K, L \triangleleft J$ and $J_{0}<J$ then

$$
N\left(K-L, J_{0}^{\prime}\right)=\mathrm{Cl}\left[N\left(K-L, J^{\prime}\right) \cap J_{0}^{\prime}-L^{\prime}\right] .
$$

Lemmas (2.12) and (2.13) follow from (2.10) and standard arguments about closures of sets in simplicial complexes.

Lemma 2.14. Suppose that $f: J_{1} \rightarrow J_{2}$ is a simplicial mapping and that $f: J_{1}^{\prime} \rightarrow J_{2}^{\prime}$ is also simplicial. If $K$ and $L$ are full subcomplexes of $J_{2}$ and $f^{-1}\left(L_{R}\right)=\left(f^{-1} L\right)_{R}$ then

(1) $f^{-1} N\left(K-L, J_{2}^{\prime}\right)=N\left(f^{-1} K-f^{-1} L, J_{1}^{\prime}\right)$,

(2) $f^{-1} \dot{N}\left(K-L, J_{2}^{\prime}\right)=\dot{N}\left(f^{-1} K-f^{-1} L, J_{1}^{\prime}\right)$.

Proof. Suppose that $A=b\left(\sigma_{0}\right) \cdots b\left(\sigma_{q}\right)<f^{-1} N\left(K-L, J_{2}^{\prime}\right)$. Then $f(A)=b\left(f \sigma_{0}\right) \cdots$ $b\left(f \sigma_{q}\right)$ is the face of a simplex of $J_{2}^{\prime}$ which meets $K^{\prime}-L^{\prime}$. Since $L_{R}^{\prime} \triangleleft K_{R}^{\prime}$ this simplex has a vertex $b(\mu)$ in $K^{\prime}-L^{\prime}$. Therefore $b(\mu) f(A)<J_{2}^{\prime}$ and there is an integer $i$ such that $f\left(\sigma_{0}\right)<\cdots<f\left(\sigma_{i}\right)<\mu<f\left(\sigma_{i+1}\right)<\cdots<f\left(\sigma_{q}\right)$. If $i<q$ there is a simplex $\bar{\mu}$ such that $\sigma_{i}<\bar{\mu}<\sigma_{i+1}$ and $f(\bar{\mu})=\mu$. Then $b(\bar{\mu}) A \cap\left(f^{-1} K-f^{-1} L\right) \neq \varnothing$ and we see that $A<N\left(f^{-1} K-f^{-1} L, J_{1}^{\prime}\right)$. If on the other hand $i=q\left(\right.$ i.e. $\left.f\left(\sigma_{q}\right)<\mu\right)$ then $f\left(\sigma_{q}\right)<K_{R}$. Hence

$$
\begin{aligned}
\sigma_{q} & <f^{-1}(\mathrm{Cl}(K-L))=f^{-1}\left((K-L) \cup L_{R}\right) \\
& =\left(f^{-1} K-f^{-1} L\right) \cup f^{-1}\left(L_{R}\right) \\
& =\left(f^{-1} K-f^{-1} L\right) \cup\left(f^{-1} L\right)_{R}, \text { by hypothesis } \\
& =\left(f^{-1} K\right)_{R}
\end{aligned}
$$

so that, $A<\sigma_{q}^{\prime}<N\left(f^{-1} K-f^{-1} L, J_{1}^{\prime}\right)$.

This proves that $f^{-1} N\left(K-L, J_{2}^{\prime}\right)<N\left(f^{-1} K-f^{-1} L, J_{1}^{\prime}\right)$. The opposite inclusion is immediate. This proves (1). Assertion (2) follows from (1) and the fact that $A \cap\left(f^{-1} K-f^{-1} L\right)=\varnothing$ if and only if $f(\sigma) \cap(K-L)=\varnothing$, q.e.d. 
Lemma 2.15. Suppose that $L<K<J$ and that $p: J \rightarrow K$ is a simplicial retraction with $p^{-1} L=L$. Then, if $p: J^{\prime} \rightarrow K^{\prime}$ is also simplicial, and if $A$ is a simplex of $L^{\prime}$

(1) $p^{-1} \dot{N}\left(L^{\prime}, K^{\prime}\right)=\dot{N}\left(L^{\prime}, J^{\prime}\right)$,

(2) $p^{-1} L k\left(A, K^{\prime}\right)=L k\left(A, J^{\prime}\right)$.

The first assertion is a special case of (2.14). The second assertion is proved by the same type of argument as was used in (2.14).

The basic example.

LEMMA 2.16. If $\sigma=\alpha \beta \gamma$ is an $n$-simplex, $\beta \neq \varnothing \neq \gamma$, and if $\sigma_{*}$ is a partition of $\sigma$ then

(a) $N\left(\alpha \beta-\alpha, \sigma_{*}^{\prime}\right)$ is a combinatorial $n$-ball. (Here $\sigma_{*}^{\prime} \equiv\left(\sigma_{*}\right)^{\prime}$.)

(b) $N\left(\alpha \beta-\alpha, \dot{\sigma}_{*}^{\prime}\right)$ and $\dot{N}\left(\alpha \beta-\alpha, \sigma_{*}^{\prime}\right)$ are $(n-1)$-balls.

(c) $\partial N\left(\alpha \beta-\alpha, \sigma_{*}^{\prime}\right)=N\left(\alpha \beta-\alpha, \dot{\sigma}_{*}^{\prime}\right) \cup \dot{N}\left(\alpha \beta-\alpha, \sigma_{*}^{\prime}\right)$.

(d) $N\left(\alpha \beta-\alpha, \dot{\sigma}_{*}^{\prime}\right)=N\left(\alpha \beta-\alpha, \sigma_{*}^{\prime}\right) \cap \dot{\sigma}_{*}^{\prime}$.

Proof. Assertion (d) is a consequence of (2.6).

Assertions (a)-(c) do not depend on which first derived $\sigma_{*}^{\prime}$ of $\sigma_{*}$ we are discussing (i.e., on which points are chosen as barycenters). Thus it will suffice to exhibit a first derived in which $\left|N\left(\alpha \beta-\alpha, \sigma_{*}^{\prime}\right)\right|$ is a convex $n$-cell, $\left|N\left(\alpha \beta-\alpha, \sigma_{*}^{\prime}\right)\right|$ is a convex $(n-1)$-cell in its boundary, and $N\left(\alpha \beta-\alpha, \dot{\sigma}_{*}^{\prime}\right)$ is the complement of $\dot{N}\left(\alpha \beta-\alpha, \sigma_{*}^{\prime}\right)$ in $\partial N\left(\alpha \beta-\alpha, \sigma_{*}^{\prime}\right)$.

Let $\eta: \sigma \rightarrow \Delta$ be the natural parametrization of $\sigma=N(\alpha \beta-\alpha, \sigma)$. Choose $\varepsilon>0$ so small that, if $v$ is a vertex of $\sigma_{*}$ not lying in $(\alpha \beta)_{*}$ and if $\eta(v)=\left(x_{1}(v), x_{2}(v)\right)$, then $\varepsilon<x_{2}(v)$. Let $b_{\varepsilon}=(1, \varepsilon)$, let $I_{\varepsilon}$ be the line segment $a b_{\varepsilon}$ and let $W_{\varepsilon}$ be the 2-simplex $a b_{0} b_{\varepsilon}$.

Suppose that $\tau$ is a simplex of $\sigma_{*}$ which does not lie in $(\alpha \beta)_{*}$. We assert

( $\uparrow: \eta^{-1} I_{\varepsilon}$ meets Int $\tau$ if and only if $\tau \cap(\alpha \beta-\alpha) \neq \varnothing$.

The proof is by analytic geometry and we leave it to the reader. This assertion allows us to choose a first derived $\sigma_{*}^{\prime}$ of $\sigma_{*}$ such that if $\tau$ is a simplex of $\sigma_{*}$ which does not lie in $(\alpha \beta)_{*}$ but which does meet $(\alpha \beta-\alpha)$, then $b(\tau) \in \eta^{-1}\left(I_{\varepsilon}\right)$. It now follows, from the linearity of $\eta$ and the assertion ( $\uparrow)$ that $\eta^{-1} W_{\varepsilon}=\left|N\left(\alpha \beta-\alpha, \sigma_{*}^{\prime}\right)\right|$ and that $\eta^{-1} I_{\varepsilon}=\left|\dot{N}\left(\alpha \beta-\alpha, \sigma_{*}^{\prime}\right)\right|$. By part (d) of the theorem we know further that $\left|N\left(\alpha \beta-\alpha, \dot{\sigma}_{*}^{\prime}\right)\right|=\left(\eta^{-1} W_{\varepsilon}\right) \cap \dot{\sigma}$.

Now, $\eta^{-1} W_{\varepsilon}$ is convex and has nonempty interior. Thus it is a combinatorial $n$-ball, which proves (a). Notice that $\eta^{-1} I_{\varepsilon}$ separates $\sigma$. Also $\eta^{-1} I_{\varepsilon}$ contains no open sets in $\sigma$ since $\eta$ is linear and $I_{\varepsilon} \cap \eta(\sigma)$ contains no open sets in $\eta(\sigma)$. Therefore $\eta^{-1} I_{\varepsilon}$ is a convex $(n-1)$-cell dividing $\sigma$ into two $n$-balls, one of which is $\eta^{-1} W_{\varepsilon}$. Thus

$$
\begin{aligned}
\partial\left(\eta^{-1} W_{\varepsilon}\right) & =\left(\eta^{-1} I_{\varepsilon}\right) \cup\left(\left(\eta^{-1} W_{\varepsilon}\right) \cap \dot{\sigma}^{\prime}\right), \\
\partial N\left(\alpha \beta-\alpha, \sigma_{*}^{\prime}\right) & =\dot{N}\left(\alpha \beta-\alpha, \sigma_{*}^{\prime}\right) \cup N\left(\alpha \beta-\alpha, \dot{\sigma}_{*}^{\prime}\right) .
\end{aligned}
$$

This proves (c). Finally (b) is true because $N\left(\alpha \beta-\alpha, \dot{\sigma}_{*}^{\prime}\right)$, as the complement of a convex $(n-1)$-cell in the boundary of a convex $n$-cell, is an $(n-1)$-ball, q.e.d. 
3. The uniqueness theorem. If $X, Y<Z$ and $(J, K, L ; h)$ is a triangulation of $(Z, X, Y)$ then $h N\left(K-L, J^{\prime \prime}\right)$ is a regular neighborhood of $X \bmod Y$ in $Z$. Thus regular neighborhoods always exist. In this section we prove the uniqueness theorem:

THEOREM 3.1. If $V$ and $W$ are regular neighborhoods of $X \bmod Y$ in $Z$ then there is an ambient isotopy $G: Z \times I \rightarrow Z \times I$ such that

(a) $G_{0}=1$,

(b) $G \mid(X \cup Y) \times I=1$,

(c) $G_{1}(V)=W$.

ADDENDUM 3.2. If $U$ is a regular neighborhood of $X \bmod Y$ in $Z$ such that $(U-Y) \subset\left(\operatorname{Int}_{z} V\right) \cap\left(\operatorname{Int}_{z} W\right)$ and if $T$ is any subpolyhedron of $Z$ such that $T \cap(V \cup W)<Y$ then $G$ may be chosen so that also $G \mid(U \cup T) \times I=1$.

The proof of (3.1) is invariant on certain subpolyhedra so that it actually yields:

ADDENDUM 3.3. If $\left(V,\left\{V_{j}\right\}\right)$ and $\left(W,\left\{W_{j}\right\}\right)$ are regular neighborhoods of $X \bmod Y$ in $\left(Z,\left\{Z_{j}\right\}\right)$ then there is an ambient isotopy $G:\left(Z,\left\{Z_{j}\right\}\right) \times I \rightarrow\left(Z,\left\{Z_{j}\right\}\right) \times I$ such that

(a) $G_{0}=1$,

(b) $G \mid(X \cup Y) \times I=1$,

(c) $G_{1}(V)=W ; G_{1}\left(V_{j}\right)=W_{j}$ for all $j$.

ADDENDUM 3.4. If $Z-(X \cup Y)$ is an n-manifold then the homeomorphism $G_{1}$ can be realized as the composition of $2 n$ moves relative to $X \cup Y$.

Here we have used the

Definition. If $Z-P$ is an $n$-manifold and $h$ is a homeomorphism of $Z$ onto itself then $h$ is a move relative to $P$ if there is a family of subpolyhedra $\left\{Q_{i}^{n}\right\}$ of $Z$ such that:

(1) The $Q_{i}^{n}$ are $n$-balls with pairwise disjoint interiors.

(2) There is a single triangulation of $Z$ in which the $Q_{i}^{n}$ are all subcomplexes.

(3) $\left(Q_{i}^{n} \cap P\right)<\partial Q_{i}^{n}$, for all $i$.

(4) $h \mid\left(Z-\bigcup_{i}\right.$ Int $\left._{z} Q_{i}^{n}\right) \cup P=1$.

(5) $h\left(Q_{i}^{n}\right)=Q_{i}^{n}$, for all $i$.

(6) If any point of $Q_{i}^{n} \cap \partial(Z-P)$ is moved under $h$ then

$$
Q_{i}^{n} \cap \mathrm{Cl}(\partial(Z-P))=Q_{i}^{n-1} \text { is a face of } Q_{i}^{n} \text { and }\left(Q_{i}^{n-1} \cap P\right)<\partial Q_{i}^{n-1} \text {. }
$$

Proof of Theorem 3.1. Let $(J, K, L ; h)$ and $(\tilde{J}, \tilde{K}, \tilde{L} ; \tilde{h})$ be full triangulations of $(Z, X, Y)$ such that $V=h N\left(K-L, J^{\prime}\right)$ and $W=\tilde{h} N\left(\tilde{K}-\tilde{L}, \tilde{J}^{\prime}\right)$. Then $\tilde{h}^{-1} h: J \rightarrow \tilde{J}$ is a piecewise linear homeomorphism, so there exist partitions $J_{*}$ and $\tilde{J}_{*}$ of $J$ and $\tilde{J}$ such that $\tilde{h}^{-1} h: J_{*} \rightarrow \tilde{J}_{*}$ is a simplicial isomorphism. Let $J_{*}^{\prime}$ and $\tilde{J}_{*}^{\prime}$ be first barycentric subdivisions. We claim that it suffices to prove the following lemma.

LemMA. If $K \triangleleft J, L \triangleleft J$ and $J_{*}$ is a partition of $J$ then there exists an ambient piecewise linear isotopy $H: J \times I \rightarrow J \times I$ such that

(1) $H_{0}=1$.

(2) $H_{t} \mid(K \cup C(K-L, J))=1(0 \leqq t \leqq 1)$. 
(3) $H_{t}(\sigma)=\sigma$ for each simplex $\sigma<J(0 \leqq t \leqq 1)$.

(4) $H_{1}\left(\left|N\left(\sigma \cap K-\sigma \cap L, \sigma_{*}^{\prime}\right)\right|\right)=\left|N\left(\sigma \cap K-\sigma \cap L, \sigma^{\prime}\right)\right|$, if $\sigma$ is a simplex of $J$ which does not lie in $K$ but meets $K-L$.

Sufficiency of the lemma. (2), (4), and (2.5) imply that $H_{t} \mid K \cup L=1$ and $H_{1} N\left(K-L, J_{*}^{\prime}\right)=N\left(K-L, J^{\prime}\right)$. Let $\tilde{H}: \tilde{J} \times I \rightarrow \tilde{J} \times I$ be the corresponding isotopy of $\tilde{J}$ given by the lemma. Then the desired isotopy $G: Z \times I \rightarrow Z \times I$ is defined as the composition of the following homeomorphisms:

$$
\begin{aligned}
&(Z \times I) \stackrel{(h \times 1)^{-1}}{\longrightarrow}(J \times I) \stackrel{H^{-1}}{\longrightarrow}(J \times I) \stackrel{(h \times I)}{\longrightarrow}(Z \times I) \\
& \stackrel{(\tilde{h} \times 1)^{-1}}{\longrightarrow}(\tilde{J} \times I) \stackrel{\tilde{H}}{\longrightarrow}(\tilde{J} \times I) \stackrel{(\tilde{h} \times 1)}{\longrightarrow}(Z \times I) .
\end{aligned}
$$

This proves the theorem modulo the lemma.

Proof of the lemma. If we can find a homeomorphism $H_{1}$ satisfying (2)-(4) then the condition (3), $H_{1}(\sigma)=\sigma$ for each $\sigma<J$, implies, by Lemma 15 of [Z], that we can find the desired isotopy. Hence our problem is to construct such an $H_{1}$.

Let $S_{i}$ be the set (not a subcomplex) of simplexes $\sigma^{j}<J(j \leqq i)$ which do not lie in $K$ but which meet $K-L$. Thus $S_{0}=\varnothing$ and, if $\operatorname{dim} J=n$, then $J=K \cup C(K-L, J)$ $\cup S_{n}$. Let $J_{i}=K \cup C(K-L, J) \cup S_{i}$. Set $f_{0}=1$. Assume inductively that a homeomorphism $f_{i}: J \rightarrow J$ has been defined such that $f_{i} \mid K \cup C(K-L, J)=1, f_{i}(\sigma)=\sigma$ for all $\sigma<J$ and $f_{i} N\left(\sigma \cap K-\sigma \cap L, \sigma_{*}^{\prime}\right)=N\left(\sigma \cap K-\sigma \cap L, \sigma^{\prime}\right)$ for all $\sigma \in S_{i}$. We proceed to define $f_{i+1}$ as a composition $g f_{i}$ for an appropriate homeomorphism $g$.

Suppose $\sigma=\sigma^{i+1} \in S_{i+1}$. By (2.9), $\sigma=\alpha \beta \gamma$ where $\alpha<L_{R}, \beta<C\left(L_{R}, K_{R}\right)$ and $\gamma<C\left(K_{R}, J\right)$. By definition of $S_{i+1}$, neither $\beta$ nor $\gamma$ is empty. Notice that

$$
(\sigma \cap K)-(\sigma \cap L)=\left(\sigma \cap K_{R}\right)-\left(\sigma \cap L_{R}\right)=\alpha \beta-\alpha .
$$

Clearly $\dot{\sigma}<J_{i}$ and $f_{i}(\dot{\sigma})=\dot{\sigma}$. By (2.16) and (2.5)

$$
\begin{aligned}
N\left(\alpha \beta-\alpha, \sigma_{*}^{\prime}\right) \cap \dot{\sigma}_{*}^{\prime} & =N\left(\alpha \beta-\alpha, \dot{\sigma}_{*}^{\prime}\right) \\
& =\bigcup\left\{N\left(\tau \cap K-\tau \cap L, \tau_{*}^{\prime}\right) \mid \tau<\dot{\sigma}, \tau \cap(K-L) \neq \varnothing\right\}
\end{aligned}
$$

and a similar statement holds for $N\left(\alpha \beta-\alpha, \sigma^{\prime}\right) \cap \dot{\sigma}^{\prime}$. Thus, applying $f_{i}$ to each summand we have

$$
f_{i}\left(N\left(\alpha \beta-\alpha, \sigma_{*}^{\prime}\right) \cap \dot{\sigma}_{*}^{\prime}\right)=N\left(\alpha \beta-\alpha, \sigma^{\prime}\right) \cap \dot{\sigma}^{\prime} .
$$

Therefore (2.16) $N\left(\alpha \beta-\alpha, \sigma^{\prime}\right)$ and $f_{i} N\left(\alpha \beta-\alpha, \sigma_{*}^{\prime}\right)$ are $(i+1)$-balls in $\sigma$ meeting $\dot{\sigma}$ in the common $i$-ball $N\left(\alpha \beta-\alpha, \dot{\sigma}^{\prime}\right)$. Recalling that $N\left(\alpha \beta-\alpha, \sigma_{*}^{\prime}\right)$ has a convex realization, it follows that $\left(\sigma, f_{i} N\left(\alpha \beta-\alpha, \sigma_{*}^{\prime}\right)\right)$ and $\left(\sigma, N\left(\alpha \beta-\alpha, \sigma^{\prime}\right)\right)$ are each homeomorphic to the cone on the pair $\left(\dot{\sigma}, N\left(\alpha \beta-\alpha, \dot{\sigma}^{\prime}\right)\right)$. Therefore there is a homeomorphism $g_{\sigma}: \sigma \rightarrow \sigma$ such that $g_{\sigma} \mid \dot{\sigma}=1$ and $g_{\sigma} f_{i} N\left(\sigma \cap K-\sigma \cap L, \sigma_{*}^{\prime}\right)=N\left(\sigma \cap K-\sigma \cap L, \sigma^{\prime}\right)$.

Notice that $N\left(b(\sigma), J^{\prime}\right)=b(\sigma) \dot{\sigma}^{\prime} \dot{D}(\sigma, J)$ where $\left|b(\sigma) \dot{\sigma}^{\prime}\right|=|\sigma|$. We define $g:|J| \rightarrow|J|$ by the conditions

(i) $g \mid N\left(b(\sigma), J^{\prime}\right)=g_{\sigma} * 1_{\dot{D}(\sigma, J)}$ if $\sigma \in\left(S_{i+1}-S_{i}\right)$,

(ii) $g(x)=x$ if $x \notin N\left(b(\sigma), J^{\prime}\right)$ for any $\sigma \in S_{i+1}-S_{i}$. 
Then, if $\sigma \in\left(S_{i+1}-S_{i}\right), g \mid \dot{N}\left(b(\sigma), J^{\prime}\right)=\left(g_{\sigma} \mid \dot{\sigma}^{\prime}\right) * 1_{\dot{D}(\sigma, J)}=1$. Also,

$$
\left[\operatorname{Int}_{|J|} N\left(b(\sigma), J^{\prime}\right)\right] \cap\left|J_{i}\right|=\varnothing,
$$

and if $\sigma \neq \tau$ then $N\left(b(\sigma), J^{\prime}\right) \cap N\left(b(\tau), J^{\prime}\right)=\dot{N}\left(b(\sigma), J^{\prime}\right) \cap \dot{N}\left(b(\tau), J^{\prime}\right)$. Therefore $g$ is a well-defined homeomorphism of $J$ onto itself, supported by

$$
\bigcup\left\{N\left(b(\sigma), J^{\prime}\right) \mid \sigma \in\left(S_{i+1}-S_{i}\right)\right\}
$$

and fixed on $J_{i}$. Finally notice that $g(\tau)=\tau$ for all $\tau<J$. For if $g(x) \neq \lambda$ then $x \in N\left(b(\sigma), J^{\prime}\right)$ for some $\sigma \in\left(S_{i+1}-S_{i}\right)$. The carrier of $x$ in $J^{\prime}$ is of the form $A B$ where $A=b\left(\sigma_{0}\right) \cdots b\left(\sigma_{q}\right)<\dot{D}(\sigma, J)$ and $b(\sigma)<B<b(\sigma) \dot{\sigma}^{\prime}$. If $A=\varnothing$ then $x \in$ Int $\sigma$ and $g(x) \in$ Int $\sigma$. If $A \neq \varnothing$ then $x \in$ Int $\sigma_{q}$ and $g(x) \in A b(\sigma) \dot{\sigma}^{\prime}$ is also in $\sigma_{q}$ since its barycentric coordinate relative to $b\left(\sigma_{q}\right)$ is unchanged. Hence $g$ does not change the carrier of any point in $J$.

If we define $f_{i+1}=g f_{i}$ and proceed inductively then the homeomorphism $f_{n} \equiv H_{1}$ satisfies conditions (2)-(4), q.e.d.

Proof of Addendum 3.2. We shall show (7.1 and 7.3) that under the hypothesis of (3.2) $V$ and $W$ are both regular neighborhoods of $U \bmod (Y \cup T)$ in $Z$. Apply (3.1).

Proof of Addendum 3.3. Condition (3) of the lemma used in the proof-namely that $H_{t}(\sigma)=\sigma$ for all $\sigma<J, 0 \leqq t \leqq 1$-implies that for any subcomplex $J_{i}<J$, $H_{t}\left(J_{i}\right)=J_{i}$ and $H_{1} N\left(K-L, J_{i^{*}}^{\prime}\right)=N\left(K-L, J_{i}^{\prime}\right)$. Thus the proof of the uniqueness theorem naturally carries within it the proof of Addendum (3.3).

Proof of Addendum 3.4. The homeomorphisms $g_{i+1}=f_{i+1} f_{i}^{-1}$ constructed in the proof of (3.1) have the properties

(1) $g_{n} \cdots g_{2} g_{1}=H_{1}$

(2) $g_{i+1} N\left(b(\sigma), J^{\prime}\right)=N\left(b(\sigma), J^{\prime}\right)$ for each $\sigma \in S_{i+1}-S_{i}$,

(3) $g_{i+1} \mid\left(J-\bigcup\left\{\operatorname{Int}_{|J|} N\left(b(\sigma), J^{\prime}\right) \mid \sigma \in S_{i+1}-S_{i}\right\}\right)=1$.

If $\sigma \in\left(S_{i+1}-S_{i}\right)$ then $\sigma \nless(K \cup L)$. So if $J-(K \cup L)$ is an $n$-manifold, it follows that $\dot{D}(\sigma, J)$ is a ball or a sphere, depending on whether or not $\sigma<\mathrm{Cl}[\partial(J-(K \cup L))]$ $\equiv J_{0}$. Then $Q^{n}(\sigma) \equiv N\left(b(\sigma), J^{\prime}\right)=b(\sigma) \dot{\sigma}^{\prime} \dot{D}(\sigma, J)$ is an $n$-ball meeting $(K \cup L)$ only in its boundary. If any point of $Q^{n}(\sigma) \cap J_{0}$ is moved by $g_{i+1}$ then $\sigma<J_{0}$ and $Q^{n}(\sigma) \cap J_{0}=$ $N\left(b(\sigma), J_{0}^{\prime}\right)$ is an $(n-1)$-ball. One checks easily now that each $g_{i+1}$ is a move. Thus $H_{1}$ is a composition of $n$ moves, $\tilde{H}_{1}$ is a composition of $n$ moves and $G_{1}$ is a composition of $2 n$ moves.

\section{The characterization of collared subpolyhedra.}

Definition. If $X<Z$ then we say that $X$ is collared in $Z$ ( $X$ is bicollared in $Z$ ) if there is a polyhedral neighborhood $V$ of $X$ in $Z$ and a homeomorphism $h: X \times[0,1]$ $\rightarrow V(h: X \times[-1,1] \rightarrow V)$ such that $h(x, 0)=x$ for all $x \in X$. We say that $X$ is locally collared in $Z$ ( $X$ is locally bicollared in $Z$ ) if, for each $x \in X$ there is a polyhedral neighborhood $V$ of $x$ in $Z$ such that $V \cap X$ is collared (bicollared) in $V$.

In this section we characterize (4.2) the collaring of $X-Y$ in $Z-Y$ when $X, Y<Z$. This result will be used in $\$ 5$ and $\$ 6$. 
LeMmA 4.1. Assume that $J_{1}$ and $J_{2}$ are complexes (in some Euclidean space) and $\sigma<L<\left(J_{1} \cap J_{2}\right)$. If there is a homeomorphism $h: J_{1} \rightarrow J_{2}$ such that $h \mid L=1$ then there is a homeomorphism $g: \operatorname{Lk}\left(\sigma, J_{1}\right) \rightarrow \operatorname{Lk}\left(\sigma, J_{2}\right)$ such that $g \mid \operatorname{Lk}(\sigma, L)=1$.

Proof. Suppose that $\sigma=v$ is a vertex of $L$. Let $J_{1 *}$ and $J_{2^{*}}$ be partitions of $J_{1}$ and $J_{2}$ such that $h: J_{1^{*}} \rightarrow J_{2^{*}}$ is a simplicial isomorphism. Then there are homeomorphisms $g_{i}: \operatorname{Lk}\left(v, J_{i^{*}}\right) \rightarrow \operatorname{Lk}\left(v, J_{i}\right),(i=1,2)$ defined by pseudo-radial projection (e.g., see L.emma 8 of $[Z])$ such that

(1) if $v<\alpha<J_{i}$ then $g_{i}\left(\left|\operatorname{Lk}\left(v, \alpha_{*}\right)\right|\right)=|\operatorname{Lk}(v, \alpha)|$,

(2) $g_{1}\left|\operatorname{Lk}\left(v, L_{*}\right)=g_{2}\right| \operatorname{Lk}\left(v, L_{*}\right)$.

So $g=g_{2} h g_{1}^{-1}: \operatorname{Lk}\left(v, J_{1}\right) \rightarrow \operatorname{Lk}\left(v, J_{2}\right)$ is a homeomorphism with $g \mid \operatorname{Lk}(v, L)=1$.

Suppose that $n>0$ and the lemma is true for all simplexes of dimension less than $n$. Let $\sigma=\sigma^{n}=v \tau<L$. Let $\bar{J}_{i}=\operatorname{Lk}\left(\tau, J_{i}\right)$ and $\bar{L}=\mathrm{Lk}(\tau, L)$. By induction hypothesis there is a homeomorphism $\bar{g}: \bar{J}_{1} \rightarrow \bar{J}_{2}$ such that $\bar{g} \mid \bar{L}=1$. Now $v$ is a vertex of $\bar{L}$, so by the case $n=0$, there is a homeomorphism $g: \operatorname{Lk}\left(v, \bar{J}_{1}\right) \rightarrow \operatorname{Lk}\left(v, \bar{J}_{2}\right)$ such that $g \mid \operatorname{Lk}(v, \bar{L})=1$. Since $\operatorname{Lk}(v, \operatorname{Lk}(\tau, T))=\operatorname{Lk}(v \tau, T)$, this completes the proof.

Proposition 4.2. If $X$ and $Y$ are subpolyhedra of $Z$, the following statements are equivalent.

(A) $X-Y$ is collared in $Z-Y$.

(B) $X-Y$ is locally collared in $Z$.

(C) If $(J, K, L ; h)$ is any triangulation of $(Z, X, Y)$ and if $\sigma$ is any simplex of $K-L$ then there is a homeomorphism $g: \operatorname{Lk}(\sigma, J) \rightarrow v \operatorname{Lk}(\sigma, K)$ such that $g \mid \operatorname{Lk}(\sigma, K)=1$.

(D) If $(J, K, L ; h)$ is a full triangulation of $(Z, X, Y)$ then there is a homeomorphism

such that

$$
g: C\left(L^{\prime}, N\left(K-L, J^{\prime}\right)\right) \rightarrow C\left(L^{\prime}, K^{\prime}\right) \times I
$$

(D.1) $g(x)=(x, 0)$ for all $x \in C\left(L^{\prime}, K^{\prime}\right)$,

(D.2) $g D(\sigma, J)=D(\sigma, K) \times I$ for all $\sigma<K-L$,

(D.3) $g\left(C_{\sigma}\right)=D(\sigma, K) \times 1$, for all $\sigma<K-L$ where $C_{\sigma} \equiv \dot{N}(D(\sigma, K), D(\sigma, J))$ $=C(\dot{D}(\sigma, K), \dot{D}(\sigma, J))\left({ }^{2}\right)$.

Proof. (A) $\Rightarrow$ (B). This is obvious.

(B) $\Rightarrow(\mathrm{C})$. Assume first that $\sigma=x$ is a vertex of $K-L$. If $K_{*}$ is any partition of $K$ then St $\left(x, K_{*}\right) \times I=P$ can be triangulated as

$$
(x, 0) *\left[\left(\operatorname{Lk}\left(x, K_{*}\right) \times I\right) \cup\left(\operatorname{St}\left(x, K_{*}\right) \times 1\right)\right] .
$$

Identify $K_{*}=K_{*} \times 0$. Obviously there is a homeomorphism

$$
g:\left(\operatorname{Lk}(x, P), \operatorname{Lk}\left(x, K_{*}\right)\right) \rightarrow\left(v \operatorname{Lk}\left(x, K_{*}\right), \operatorname{Lk}\left(x, K_{*}\right)\right)
$$

(2) Using (7.5) one can further prove that $N\left(K-L, J^{\prime}\right)$ is (p.l.) homeomorphic to a regular neighborhood of $X_{R} \times 0 \bmod Y_{R} \times 0$ in $X_{R} \times I$ and is topologically equivalent to $\left(X_{R} \times I\right) /[(y, 0)$ $=(y, t)$ if $y \in Y_{R}$ and $\left.t \in I\right]$. 
such that $g \mid \operatorname{Lk}\left(x, K_{*}\right)=1$. Since $K-L$ is locally collared in $J$ at $x$ and since the pair $(\operatorname{Lk}(x, J), \operatorname{Lk}(x, K)) \simeq\left(\operatorname{Lk}\left(x, J_{*}\right), \operatorname{Lk}\left(x, K_{*}\right)\right)$ for any partition $\left(J_{*}, K_{*}\right)$ of $(J, K)$ it follows easily that there is a homeomorphism $g: \operatorname{Lk}(x, J) \rightarrow v \operatorname{Lk}(x, K)$ such that $g \mid \operatorname{Lk}(x, K)=1$.

If $\sigma$ is an arbitrary simplex of $K-L$ we star $J$ at $b(\sigma)$ and apply the above result to $b(\sigma)$. Thus there is a homeomorphism $g: \dot{\sigma} \mathrm{Lk}(\sigma, J) \rightarrow v \dot{\sigma} \mathrm{Lk}(\sigma, K)$ such that $g \mid \dot{\sigma} \mathrm{Lk}(\sigma, K)=1$. We extend this homeomorphism conewise to a homeomorphism $g_{1}: \sigma \operatorname{Lk}(\sigma, J)=v \sigma \operatorname{Lk}(\sigma, K)$ such that $g_{1} \mid \sigma \operatorname{Lk}(\sigma, K)=1$. Finally we apply (4:1) and get the desired homeomorphism $g_{2}: \operatorname{Lk}(\sigma, J) \rightarrow v \operatorname{Lk}(\sigma, K)$.

(C) $\Rightarrow$ (D). We define the homeomorphism $g$ inductively. Let

$$
\begin{aligned}
& J_{i}=\bigcup\left\{D\left(\sigma^{n-i}, J\right) \mid \sigma^{n-i}<K-L\right\}<J^{\prime}, \\
& K_{i}=\bigcup\left\{D\left(\sigma^{n-i}, K\right) \mid \sigma^{n-i}<K-L\right\}<K^{\prime},
\end{aligned}
$$

where $n$ is the dimension of $K-L$. Notice (see (2.10)) that $J_{n}=C\left(L^{\prime}, N\left(K-L, J^{\prime}\right)\right)$ and $K_{n}=C\left(L^{\prime}, K^{\prime}\right)$. Suppose that a homeomorphism $g_{i-1}: J_{i-1} \rightarrow K_{i-1} \times I$ has been constructed to satisfy (D.1)-(D.3) for all simplexes of dimension greater than $(n-i)$. Let $\sigma=\sigma^{n-i}<K-L$. Notice that

$$
\begin{aligned}
D(\sigma, J) \cap J_{i-1} & =\bigcup\{D(\tau, J) \mid \sigma \supsetneqq \tau<K-L\}=N(\dot{D}(\sigma, K), \dot{D}(\sigma, J)), \\
\dot{D}(\sigma, K) & =\bigcup\{D(\tau, K) \mid \sigma \supsetneqq \tau<K-L\}
\end{aligned}
$$

and

$$
\dot{N}(\dot{D}(\sigma, K), \dot{D}(\sigma, J))=\bigcup\left\{C_{\tau} \mid \sigma \supsetneqq \tau<K-L\right\} .
$$

Hence, using (D.1)-(D.3), we have

and

$$
g_{i-1}\left(D(\sigma, J) \cap J_{i-1}^{\cdot}\right)=\dot{D}(\sigma, K) \times I, \quad g_{i-1} \mid \dot{D}(\sigma, K)=1
$$

$$
g_{i-1} \dot{N}(\dot{D}(\sigma, K), \dot{D}(\sigma, J))=\dot{D}(\sigma, K) \times 1 .
$$

We first extend $g_{i-1}$ over $C_{\sigma}$. The natural isomorphism of $(\dot{D}(\sigma, J), \dot{D}(\sigma, K))$ onto (Lk $\left.(\sigma, J)^{\prime}, \mathrm{Lk}(\sigma, K)^{\prime}\right)$ takes $N(\dot{D}(\sigma, K), \dot{D}(\sigma, J))$ onto the regular neighborhood $N\left(\operatorname{Lk}(\sigma, K)^{\prime}, \operatorname{Lk}(\sigma, J)^{\prime}\right)$ of $\operatorname{Lk}(\sigma, K)$ in $\operatorname{Lk}(\sigma, J)$. But by $(\mathrm{C}), \operatorname{Lk}(\sigma, J) \simeq v \operatorname{Lk}(\sigma, K)$ and we know that the complement of a regular neighborhood of $T$ in $v T$ is just a smaller concentric cone. Thus $C_{\sigma}$ is piecewise linearly a cone on its subcomplex $\dot{N}(\dot{D}(\sigma, K), \dot{D}(\sigma, J))$. On the other hand, $D(\sigma, K) \times 1=(b(\sigma), 1) *(\dot{D}(\sigma, K) \times 1)$. Thus we may extend $g_{i-1}$ conewise over $C_{\sigma}$ and obtain a homeomorphism of $\dot{D}(\sigma, J)$ onto $(\dot{D}(\sigma, K) \times I) \cup(D(\sigma, K) \times 1)$. Finally we notice that $D(\sigma, J)$ $=b(\sigma) \dot{D}(\sigma, J)$ and the cell complex $D(\sigma, K) \times l$ can be triangulated as $(b(\sigma), 0) *$ $[(\dot{D}(\sigma, K) \times I) \cup(D(\sigma, K) \times 1)]$. Thus we may extend $g_{i-1}$ conewise to all of $D(\sigma, J)$. Doing this simultaneously for all $\sigma^{n-i}$ we construct a homeomorphism $g_{i}$ satisfying (D.1)-(D.3). Finally we set $g=g_{n}$.

(D) $\Rightarrow$ (B). Suppose $x \in X-Y$. Choose a full triangulation $(J, K, L ; h)$ of $(Z, X, Y)$ in which $v=h^{-1}(x)$ is a vertex. Then by (D), $(D(v, J), D(v, K)) \simeq$ $(D(v, K) \times I, D(v, K) \times 0)$. Hence $X$ is locally collared in $Z$ at $x=h(v)$. 
(D) $\Rightarrow$ (A). Let $\tilde{Z}=Z-Y, \tilde{X}=X-Y$ and $\tilde{Y}=\varnothing$. Let $(\tilde{\mathrm{A}})-(\tilde{\mathrm{D}})$ be the assertions analogous to (A)-(D). Then (D) $\Rightarrow(\mathrm{B}) \Rightarrow(\tilde{\mathrm{B}}) \Rightarrow(\tilde{\mathrm{D}})$. However $(\tilde{\mathrm{D}})$ tells us that $C\left(\tilde{L}, N\left(\tilde{K}-\tilde{L}, \tilde{J}^{\prime}\right)\right)=N\left(\tilde{K}^{\prime}, \tilde{J}^{\prime}\right)$ is a collar of $\tilde{K}$. Thus $\tilde{X}=X-Y$ is collared in $\tilde{Z}=Z-Y$, q.e.d.

As a consequence of (4.2) we get the following "join-cobordism theorem for polyhedra." This is a generalization of Theorem (10.3) of [C].

CoRollary 4.3. Suppose that $K$ and $L$ are disjoint, nonempty subcomplexes of $J$, that each of these complexes is locally collared in $J$ and that every simplex in $J$ is the join of a simplex in $K$ and a simplex in L. Then there is a homeomorphism $h:(J, K, L)$ $\rightarrow(K \times I, K \times 0, K \times 1)$.

Proof. By (4.2D) there is a homeomorphism $f: N\left(K^{\prime}, J^{\prime}\right) \rightarrow K^{\prime} \times I$ such that $f\left(K^{\prime}\right)=K^{\prime} \times 0$ and $f \dot{N}\left(K^{\prime}, J^{\prime}\right)=K^{\prime} \times 1$. Similarly there is a homeomorphism $g$ of $N\left(L^{\prime}, J^{\prime}\right)$ onto $L^{\prime} \times I$ such that $g\left(L^{\prime}\right)=L^{\prime} \times 1$ and $g \dot{N}\left(L^{\prime}, J^{\prime}\right)=L^{\prime} \times 0$. Define $h: J \rightarrow K \times[0,2]$ by stipulating that $h \mid N\left(K^{\prime}, J^{\prime}\right)=f$ and $h\left(g^{-1}(x, t)\right)=\left(h g^{-1}(x, 0)\right)$ $+(0, t)$, where $K<R^{q}$ and $(0, t) \in R^{q} \times I$. This is a well-defined homeomorphism because $\dot{N}\left(K^{\prime}, J^{\prime}\right)=\dot{N}\left(L^{\prime}, J^{\prime}\right)$, q.e.d.

\section{Cone-retracting and collaring regular neighborhoods.}

THEOREM 5.1. If $V$ is a regular neighborhood of $X \bmod Y$ in $Z$ then there is a retraction $p: V \rightarrow X_{R}$ such that

(a) If $x \in X-Y$ then $p^{-1}(x)$ is a compact cone with $x$ as cone point and $p^{-1}(x) \cap$ $\mathrm{Bdy}_{z} V$ as base.

(b) $p^{-1}\left(Y_{R}\right)=Y_{R}$.

AdDENDUM 5.2. If $X-Y$ is a proper locally unknotted n-manifold in the $q$ manifold $Z-Y$ then the projection may be chosen so that, for each $x \in X-Y$, the base of the cone $p^{-1}(x)$ is homeomorphic to $S^{q-n-1} \times B^{i}$ where $i=i(x)$ is an integer depending on $x$.

Proof of (5.1). It suffices (see (2.8) and (2.12)) to prove that, if $L \triangleleft K \triangleleft J$ $=N(K-L, J)$, then there is a retraction $p$ of $N\left(K-L, J^{\prime}\right)$ onto $K^{\prime}$ such that $p^{-1}(x)=x$ if $x \in L$ and $p^{-1}(x)$ is a compact p.l. cone on $p^{-1}(x) \cap \dot{N}\left(K-L, J^{\prime}\right)$ otherwise.

Let $\dot{N}=\dot{N}(K, J)$. We may assume without loss of generality that $K<\left(R^{n} \times 0\right)$ and $v \dot{N}<\left(0 \times R^{p}\right)$ are complexes in $R^{n+p}=R^{n} \times R^{p}$, where $v=(0,0)$ is thought of as the origin in $R^{p}$. Consider the polyhedron $Q<R^{n+p}$ given by

$$
Q=\bigcup\{\alpha *(\beta \times v \gamma) \mid \alpha \beta \gamma<J=N(K-L, J)\}
$$

Here $\alpha *(\beta \times v \gamma)$ is the convex hull of the simplex $\alpha$ and the convex cell $(\beta \times v \gamma)$. It is the union of all line segments from points of $\alpha$ to points of $(\beta \times v \gamma)$. (Indeed if $S$ is a complex partitioning $(\beta \times v \gamma)$ then $\alpha$ and $S$ are joinable.) If $\alpha=\varnothing$ then $\alpha *(\beta \times v \gamma)$ 
$\equiv(\beta \times v \gamma)$. Let $\bar{q}: R^{n} \times R^{p} \rightarrow R^{n}$ be the natural projection. Let $q=\bar{q} \mid Q$. Then, if $\alpha \beta \gamma<J$,

$$
\begin{aligned}
q(\alpha *(\beta \times v \gamma)) & =q\{t(a, 0)+(1-t)(b, c) \mid(a, b, c, t) \in(\alpha \times \beta \times v \gamma \times I)\} \\
& =\{t(a, 0)+(1-t)(b, 0) \mid(a, b, t) \in(\alpha \times \beta \times I)\} \\
& =\alpha * \beta .
\end{aligned}
$$

Thus $q$ is a retraction of $Q$ onto $K$. Clearly $q^{-1} L=L$. If $x$ is a point of $K-L$ with carrier $\alpha_{0} \beta_{0}$, say $x=t a+(1-t) b$, then

$$
\begin{aligned}
q^{-1}(x) & =\left\{t(a, 0)+(1-t)(b, c) \mid c \in v \gamma, \alpha_{0} \beta_{0} \gamma<J\right\} \\
& =t(a, 0)+(1-t)\left[b \times v *\left(\operatorname{Lk}\left(\alpha_{0} \beta_{0}, J\right) \cap \dot{N}\right)\right] .
\end{aligned}
$$

Therefore $\left(q^{-1}(x), q^{-1}(x) \cap \bigcup\{\alpha *(\beta \times \gamma) \mid \alpha \beta \gamma<J\}\right) \simeq(v P, P)$ where

$$
P=\operatorname{Lk}\left(\alpha_{0} \beta_{0}, J\right) \cap \dot{N}(K, J) \text {. }
$$

Let us define a cell complex to consist of a polyhedron $T$ together with a covering family of subpolyhedra-each a piecewise linear ball-such that the interiors of these balls are pairwise disjoint, the family is closed under finite intersection, and the boundary of each of these balls is the union of balls in the family. If two cell complexes are isomorphic in the sense that there is an incidence preserving one-one correspondence between the covering families then one can easily build a piecewise linear homeomorphism between the underlying polyhedra which carries each cell onto its corresponding cell.

Now $Q$ is a cell complex with typical cells of the form $\alpha *(\beta \times v \gamma)$ or $\alpha *(\beta \times \gamma)$ or $\alpha \beta$, where $\alpha \beta \gamma$ is an arbitrary simplex of $N(K-L, J)$. Similarly (see (2.16)) $N\left(K-L, J^{\prime}\right)$ is a cell-complex with typical cells of the form $N\left(\alpha \beta-\alpha,(\alpha \beta \gamma)^{\prime}\right)$ or $\dot{N}\left(\alpha \beta-\alpha,(\alpha \beta \gamma)^{\prime}\right)$ or $(\alpha \beta)$ where $\alpha \beta \gamma<N(K-L, J)$. The obvious isomorphism between these complexes induces a homeomorphism $h:\left(N\left(K-L, J^{\prime}\right), K\right) \rightarrow(Q, K)$ which is the identity on $K$ and takes $\left(N\left(K-L, J^{\prime}\right)\right)$ onto

$$
\bigcup\{\alpha *(\beta \times \gamma) \mid \alpha \beta \gamma<N(K-L, J)\} \text {. }
$$

Then $p=h^{-1} q h$ has the desired properties, q.e.d.

Proof of (5.2). Suppose that $(J, K, L ; h)$ is a full triangulation of $(Z, X, Y)$ with $V=h N\left(K-L, J^{\prime}\right)$. Let $x \in(X-Y)$ and let $\alpha \beta$ be the carrier of $h^{-1}(x)$ in $J$. The proof of (5.1) shows that the projection $p: V \rightarrow X_{R}$, determined by this triangulation, has the property that $p^{-1}(x)$ is the cone on $P$ where

$$
P \simeq \operatorname{Lk}(\alpha \beta, N(K-L, J)) \cap \dot{N}\left(K_{R}, N(K-L, J)\right) .
$$

Assume now that we had chosen $(J, K, L ; h)=\left(J_{0}^{\prime}, K_{0}^{\prime}, L_{0}^{\prime} ; h\right)$ where $\left(J_{0}, K_{0}, L_{0} ; h\right)$ was already a full triangulation of $(Z, X, Y)$. Thus $\alpha \beta=b\left(A_{0} B_{0}\right) \cdots b\left(A_{r} B_{r}\right)$ where $A_{i}<\left(L_{0}\right)_{R}, B_{i}<C\left(L_{0}, K_{0}\right)$ and $B_{r} \neq \varnothing$. So

$$
\begin{aligned}
P & \simeq\left\{b\left(A_{r} B_{r} D_{0}\right) \cdots b\left(A_{r} B_{r} D_{q}\right) \mid D_{i}<\left(\operatorname{Lk}\left(A_{r} B_{r}, J_{0}\right)-\operatorname{Lk}\left(A_{r} B_{r}, K_{0}\right)\right)\right\} \\
& \simeq C\left(\operatorname{Lk}\left(A_{r} B_{r}, K\right)^{\prime}, \operatorname{Lk}\left(A_{r} B_{r}, J\right)^{\prime}\right) .
\end{aligned}
$$


Since $(Z-Y, X-Y)$ is a proper locally unknotted manifold pair, this is homeomorphic to the complement of the regular neighborhood of $B^{n-j-1}$ in $B^{q-j-1}$, or of $S^{n-j-1}$ in $S^{q-j-1}$, where $j$ is the dimension of $A_{r} B_{r}$, q.e.d.

THEOREM 5.3. If $V$ is a regular neighborhood of $X$ mod $Y$ in $Z$ then $\left(\operatorname{Bdy}_{Z} V\right)-Y$ is bicollared in $Z-(X \cup Y)$.

COROLLARY 5.4. If $(Z-Y)$ is an n-manifold and $V$ is a regular neighborhood of $X \bmod Y$ in $Z$ then $(V-Y)$ and $(Z-V)-Y$ are n-manifolds. The second conclusion holds under the weaker assumption that $Z-(X \cup Y)$ is an n-manifold.

REMARK. When $Z$ is a manifold there are certain important pairs of polyhedra $X, Y$-e.g. the link-collapsible pairs studied in [H-Z]-for which $V$ itself is a manifold.

Proof of (5.3). Let $(J, K, L ; h)$ be a full triangulation of $(Z, X, Y)$ such that $V=h N\left(K-L, J^{\prime}\right)$. We shall use (4.2c) to show that $\dot{N}\left(K-L, J^{\prime}\right)-L^{\prime}$ is collared in $C\left(K-L, J^{\prime}\right)-L^{\prime}$ and in $N\left(K-L, J^{\prime}\right)-\left(K^{\prime} \cup L^{\prime}\right)$. This is sufficient by (2.12).

Let $A$ be a simplex of $\dot{N}\left(K-L, J^{\prime}\right)-L^{\prime}$. Then $A=b\left(\sigma_{0}\right) \cdots b\left(\sigma_{q}\right)$ where $\sigma_{i}=\alpha_{i} \beta_{i} \gamma_{i}$ $<N(K-L, J)$. Moreover $\beta_{q} \neq \varnothing \neq \gamma_{q}$, and $\beta_{i}$ is empty if and only if $\gamma_{i}$ is empty. Hence $A=b\left(\alpha_{0}\right) \cdots b\left(\alpha_{r-1}\right) b\left(\sigma_{r}\right) \cdots b\left(\sigma_{q}\right)$ where $\beta_{r} \neq \varnothing \neq \gamma_{r}$. Decomposing Lk $\left(A, J^{\prime}\right)$ into the blocks determined by its vertices we have

$$
\begin{aligned}
\operatorname{Lk}\left(A, J^{\prime}\right) & =\operatorname{Lk}\left(b\left(\alpha_{0}\right) \cdots b\left(\alpha_{r-1}\right) b\left(\sigma_{r}\right), \sigma_{r}^{\prime}\right) * \dot{D}\left(\sigma_{r}, \dot{\sigma}_{r+1}\right) \cdots \dot{D}\left(\sigma_{q-1}, \dot{\sigma}_{q}\right) * \dot{D}\left(\sigma_{q}, J\right) \\
& =\operatorname{Lk}\left(B, \sigma_{r}^{\prime}\right) * S
\end{aligned}
$$

where $B=b\left(\alpha_{0}\right) \cdots b\left(\alpha_{r-1}\right) b\left(\sigma_{r}\right)$ and $S=\dot{D}\left(\sigma_{r}, \dot{\sigma}_{r+1}\right) \cdots \dot{D}\left(\sigma_{q}, J\right)$.

We assert that

(1) $\operatorname{Lk}\left(A, \dot{N}\left(K-L, J^{\prime}\right)\right)=\operatorname{Lk}\left(B, \dot{N}\left(\alpha_{r} \beta_{r}-\alpha_{r}, \sigma_{r}^{\prime}\right)\right) * S$,

(2) $\operatorname{Lk}\left(A, N\left(K-L, J^{\prime}\right)\right)=\operatorname{Lk}\left(B, N\left(\alpha_{r} \beta_{r}-\alpha_{r}, \sigma_{r}^{\prime}\right)\right) * S$,

(3) $\operatorname{Lk}\left(A, C\left(K-L, J^{\prime}\right)\right)=\operatorname{Lk}\left(B, C\left(\alpha_{r} \beta_{r}-\alpha_{r}, \sigma_{r}^{\prime}\right)\right) * S$.

Let $N_{r}=N\left(\alpha_{r} \beta_{r}-\alpha_{r}, \sigma_{r}^{\prime}\right)$ and $C_{r}=C\left(\alpha_{r} \beta_{r}-\alpha_{r}, \sigma_{r}^{\prime}\right)$. Consider the first assertion. Every simplex in $S$ lies in $\dot{N}\left(K-L, J^{\prime}\right)$ because, for each vertex $b\left(\sigma_{i}\right)=b\left(\alpha_{i} \beta_{i} \gamma_{i}\right)$ with $i>r, \beta_{i} \neq \varnothing \neq \gamma_{i}$. Also $\dot{N}_{r}<\dot{N}\left(K-L, J^{\prime}\right)$. Hence, the right-hand side of (1) is contained in $\dot{N}\left(K-L, J^{\prime}\right) \cap \mathrm{Lk}\left(A, J^{\prime}\right)$. This intersection is equal to $\operatorname{Lk}\left(A, \dot{N}\left(K-L, J^{\prime}\right)\right)$ because $\dot{N}\left(K-L, J^{\prime}\right)$ is full in $J^{\prime}$. Therefore the right-hand term is contained in the left-hand term. On the other hand, a simplex in this intersection is of the form $D_{1} D_{2}$ where $D_{1}<\operatorname{Lk}\left(B, \sigma_{r}^{\prime}\right), \quad D_{2}<S$ and $D_{1}<\left[\dot{N}\left(K-L, J^{\prime}\right) \cap \sigma_{r}^{\prime}\right]=\dot{N}_{r}$. Therefore $D_{1}$ $<\operatorname{Lk}\left(B, \sigma_{r}^{\prime}\right) \cap \dot{N}_{r}=\operatorname{Lk}\left(B, \dot{N}_{r}\right)$. Thus $D_{1} D_{2}$ is a simplex of the right-hand side. This proves (1). Assertions (2) and (3) are proved similarly.

We wish to show that $\operatorname{Lk}\left(A, N\left(K-L, J^{\prime}\right)\right)$ is piecewise linearly a cone on Lk $\left(A, \dot{N}\left(K-L, J^{\prime}\right)\right)$. Using (1) and (2) it will suffice to show that $\mathrm{Lk}\left(B, N_{r}\right)$ is a cone on $\mathrm{Lk}\left(B, \dot{N}_{r}\right)$. Now $N_{r}$ is a ball and $\dot{N}_{r}$ is a face of this ball by (2.16). Since the vertex $b\left(\sigma_{r}\right)$ of $B$ lies in the interior of $\dot{N}_{r}$ we see that $\operatorname{Lk}\left(B, \dot{N}_{r}\right)=\operatorname{Lk}\left(B, \partial N_{r}\right)$ $=\partial \operatorname{Lk}\left(B, N_{r}\right)$. Therefore $\operatorname{Lk}\left(B, N_{r}\right)$ is homeomorphic to $v \operatorname{Lk}\left(B, \dot{N}_{r}\right)$ by a 
homeomorphism which is the identity on $\mathrm{Lk}\left(B, \dot{N}_{r}\right)$. This completes the proof that $\left(\mathrm{Bdy}_{z} V\right)-Y$ is collared in $V-(X \cup Y)$.

One proves similarly that $\left(\mathrm{Bdy}_{z} V\right)-Y$ is collared in $\mathrm{Cl}((Z-V))-Y$, q.e.d.

Proof of (5.4). If, in the preceding proof, $J-L$ is an $n$-manifold then $\dot{D}\left(\sigma_{q}, J\right)$ $\cong \operatorname{Lk}\left(\sigma_{q}, J\right)^{\prime}$ is a ball or a sphere, for each $\sigma_{q}<J-L$. Thus formulas (1)-(3) imply that $\left(N\left(K-L, J^{\prime}\right)-L^{\prime}\right)$ and $\left(C\left(K-L, J^{\prime}\right)-L^{\prime}\right)$ are $n$-manifolds, q.e.d.

EXAMPLE 5.5. One might hope that regular neighborhoods could be characterized by Theorems (5.1) and (5.3). This is not possible, as the following example shows. (The matter is further discussed in $\$ 9$.)

Let $D^{3}=[-1,1]^{3}<R^{3}$ and let $A$ be a polygonal arc which consists of the straight line from $(-1,0,0)$ to $\left(\frac{1}{2}, 0,0\right)$ plus a knotted arc from $\left(\frac{1}{2}, 0,0\right)$ to $\left(\frac{3}{4}, 0,0\right)$, contained in $\left[\frac{1}{2}, \frac{3}{4}\right] \times\left[-\frac{1}{2}, \frac{1}{2}\right]^{2}$, plus the straight line from $\left(\frac{3}{4}, 0,0\right)$ to $(1,0,0)$. Let $Z=D^{3} \cup(A \times I)<R^{4}, X=(0,0,0) \times I$ and $Y=\varnothing$. Let $V_{t}=\left([-t, t]^{3} \times I\right) \cap Z$ for $t=\frac{1}{4}, t=\frac{3}{4}$. Then $\mathrm{Bdy}_{z} V_{t}$ is bicollared and the projection map $p: V_{t} \rightarrow X$ given by $p(x, y, z, w)=(0,0,0, w)$ yields point inverses which are either arcs or the 3-ball $\left([-t, t]^{3} \times 0\right)$. Obviously $V_{1 / 4}$ is a regular neighborhood of $X$ in $Z$. But $V_{3 / 4}$ is not such a regular neighborhood, since any homeomorphism of $V_{3 / 4}$ onto $V_{1 / 4}$ would have to take the knotted ball pair $\left(\left[-\frac{3}{4}, \frac{3}{4}\right]^{3}, A \cap\left[-\frac{3}{4}, \frac{3}{4}\right]^{3}\right)$ onto the unknotted ball pair $\left(\left[-\frac{1}{4}, \frac{1}{4}\right]^{3},\left[-\frac{1}{4}, \frac{1}{4}\right] \times 0 \times 0\right)$.

\section{The stellar neighborhood theorem.}

Definition. If $Y<X<Z$ then a stellar triangulation of $(Z, X, Y)$ is a full triangulation $(J, K, L ; h)$ such that $J=N(K-L, J)$.

The purpose of this section is to prove

Theorem 6.1 (The STellar neighborhood theorem). Suppose that $X, Y, V<Z$ where $V$ is a topological neighborhood of $X-Y$ and $V \cap Y=Y_{R}$. Then

(a) If there is a stellar triangulation $(J, K, L ; h)$ of $\left(V, X_{R}, Y_{R}\right)$ such that $h(\dot{N}(K-L, J)-L)$ is locally collared in $V$ then there are regular neighborhoods $U$ and $U_{0}$ of $X \bmod Y$ in $Z$, contained in $V$, and there is an isotopy $H$ of $V$ into itself such that $H \mid(V \times 0) \cup\left(U_{0} \times I\right)=1$ and $H_{1}(V)=U$.

(b) If $\left(\mathrm{Bdy}_{Z} V\right)-Y$ is locally bicollared in $Z$ and if there is a stellar triangulation $(J, K, L ; h)$ of $\left(V, X_{R}, Y_{R}\right)$ such that $h \dot{N}(K-L, J)=\left(\mathrm{Bdy}_{z} V\right) \cup Y_{R}$ then $V$ is a regular neighborhood of $X \bmod Y$ in $Z$.

(c) If there is a full triangulation $(J, K, L ; h)$ of $(Z, X, Y)$ such that $h N(K-L, J)$ $=V$ and $\dot{N}(K-L, J)-L$ is locally collared in $N(K-L, J)$ and in $C(K-L, J)$ then $V$ is a regular neighborhood of $X \bmod Y$ in $Z$.

REMARK 1. Surprisingly, it is not enough to assume in (c) that $S=(\dot{N}(K-L, J)-L)$ is bicollared in $J$. This is illustrated when $L=\varnothing, K=\{a, b\}$ and $J=a c d \cup b c d$. The problem is that $S$ may be bicollared and yet not be contained in $\left(\mathrm{Bdy}_{z} V\right)-Y$. However, it is easy to show that if $S$ is locally bicollared and contained in $\left(\mathrm{Bdy}_{z} V\right)-Y$ then $S$ is locally collared in $N(K-L, J)$ and in $C(K-L, J)$. 
REMARK 2. The proof will be rather long because of technical difficulties resulting when $Y \neq \varnothing$. However, the basic idea of the general proof is given when $Y=\varnothing$ by the following observation: If $K \triangleleft J=N(K, J)$ and if $\dot{N}(K, J)$ is locally collared in $J$ then $N(K, J)^{\prime}$ is the union of a first derived neighborhood of $K$ and a collar.

We first prove two preliminary lemmas.

LEMMA 6.2. Suppose that $f: \sigma \rightarrow \tau$ is a simplicial mapping. Let $\tau^{\prime}$ be the standard barycentric subdivision of $\tau$. Let $\sigma^{\prime}$ be the first derived of $\sigma$ determined by the condition that $b(\alpha)$ is the centroid of the convex cell $(f \mid \alpha)^{-1} b(f(\alpha))$, for each $\alpha<\sigma$. Then $D(\rho, \sigma)$ is convex for each $\rho<\sigma$.

Proof. If $\rho=x_{0} \cdots x_{m}$ then $D(\rho, \sigma)=\bigcap\left\{D\left(x_{i}, \sigma\right) \mid 0 \leqq i \leqq m\right\}$. Therefore it suffices to show that $D(v, \sigma)$ is convex for each vertex $v$.

Let $\tau=u_{0} \cdots u_{q}$ and let $f^{-1}\left(u_{i}\right)=v_{0}^{i} v_{1}^{i} \cdots v_{n_{i}}^{i}=\sigma_{i}$. (We may assume that $f$ is onto, so $f^{-1}\left(u_{i}\right) \neq \varnothing$.) Set $\sigma=v_{0} \cdots v_{n}$ where the $v$ 's are indexed so that

$$
v_{j}^{i}=v_{\left(n_{0}+1\right)+\cdots+\left(n_{i-1}+1\right)+j} \quad\left(n_{-1} \equiv-1\right) .
$$

Let $\lambda_{k}(x)$ or $\lambda_{j}^{i}(x)$ be the barycentric coordinate of $x \in \sigma$ with respect to $v_{k}$ or $v_{j}^{i}$ respectively. Notice-or if desired take as definition - the fact that the centroid of $f^{-1} b(\tau)$ is given by

$$
\text { [centroid of } \begin{aligned}
\left.f^{-1} b(\tau)\right] \equiv b(\sigma) & =\frac{1}{q+1} \sum_{j=0}^{q} b\left(\sigma_{j}\right) \\
& =\frac{1}{q+1} \sum_{j=0}^{q}\left(\frac{v_{0}^{j}+v_{1}^{j}+\cdots+v_{n j}^{j}}{n_{j}+1}\right) .
\end{aligned}
$$

A similar formula holds for the barycenter of each face of $\sigma$.

We prove that $D\left(v_{0}, \sigma\right)$ is convex by exhibiting it as the intersection of $\sigma$ with a family of half-spaces. Through each 1-simplex $v_{0} v_{j}$ of $\sigma$ we define a hyperplane $H_{j}$ bisecting $v_{0} v_{j}$. This in turn determines the appropriate half-space $H_{j}^{+}$containing $v_{0}$. If $1 \leqq j \leqq\left(n_{0}+1\right)$ then the defining equations are:

Hyperplane $H_{j}: \lambda_{0}=\lambda_{j}$.

Half-space $H_{j}^{+}: \lambda_{0} \geqq \lambda_{j}$.

(NoTE. $H_{j}$ is the plane generated by $b\left(v_{0} v_{j}\right) * \operatorname{Lk}\left(v_{0} v_{j}, \sigma\right)$.) If $j>\left(n_{0}+1\right)$ and $v_{j}=v_{k}^{i}$ then the appropriate equations are:

Hyperplane $H_{j}: \lambda_{0}^{0}+\cdots+\lambda_{n_{0}}^{0}=\lambda_{0}^{i}+\cdots+\lambda_{n_{i}}^{i}$.

Half-space $H_{j}^{+}: \lambda_{0}^{0}+\cdots+\lambda_{n_{0}}^{0} \geqq \lambda_{0}^{i}+\cdots+\lambda_{n_{i}}^{i}$.

(NoTE. $H_{j}$ is the plane generated by [middle slice of $\sigma_{0} * \sigma_{i}$ ] $* \operatorname{Lk}\left(\sigma_{0} \sigma_{i}, \sigma\right)$ )

To show that $\left|N\left(v_{0}, \sigma^{\prime}\right)\right|$ is included in the convex set $\bigcap_{j}\left(H_{j}^{+} \cap \sigma\right)$ it suffices to prove that each vertex of $N\left(v_{0}, \sigma^{\prime}\right)$ is in this intersection. This is a straightforward and tedious exercise in barycentric coordinates using the formula for centroids given above. To demonstrate the reverse inclusion one considers a point $x$ in $\left(\sigma-\left|N\left(v_{0}, \sigma^{\prime}\right)\right|\right)$. The carrier of $x$ in $\sigma^{\prime}$ is a simplex of the form $b\left(\alpha_{0}\right) \cdots b\left(\alpha_{s}\right)$ where 
$v_{0} \nless \alpha_{0}$ (and, as always, $\alpha_{0}<\alpha_{1}<\cdots<\alpha_{s}$ ). A calculation of barycentric coordinates proves that there is an $H_{j}$ such that $x \notin H_{j}^{+}$, q.e.d.

LemMA 6.3. Assume that $\Delta=a b_{0} b_{4}$ is the 2-simplex pictured below.

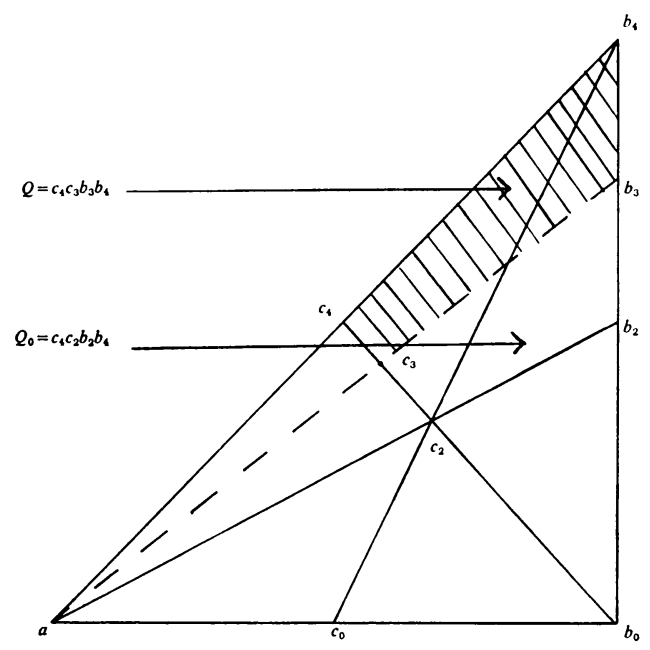

FIGURE 1

Let $\Delta^{\prime}$ be the barycentric subdivision of $\Delta$. Assume that $\eta: \psi \beta \rightarrow \Delta$ is a simplicial mapping such that $b_{4}<\eta(\psi)<a b_{4}$ and $\eta(\beta)<b_{0}$. $\left(\beta\right.$ may be empty.) Let $(\psi \beta)^{\prime}$ be the first derived of $\psi \beta$ chosen so that $b(\alpha)$ is the centroid of $(\eta \mid \alpha)^{-1} b(\eta(\alpha))$, for each $\alpha<\psi \beta$. Let $(\psi \beta)^{*}$ be the first derived of $\psi \beta$ chosen so that the barycenter of $\alpha$, denoted $b^{*}(\alpha)$, is defined as follows: (see Figure 1)

(i) If $\eta(\alpha)<a b_{0} \cup a b_{4}$ then $b^{*}(\alpha)=b(\alpha)$.

(ii) If $\alpha=\rho \beta_{0}$ where $\rho<\psi, \varnothing \neq \beta_{0}<\beta$ and $b_{4}<\eta(\rho)$ then

$$
b^{*}(\alpha)=\eta^{-1}\left(c_{3} b_{3}\right) \cap b(\rho) b\left(\rho \beta_{0}\right) .
$$

Let $D(, \quad)$ and $D^{*}(, \quad)$ denote duals taken with respect to $(\psi \beta)^{\prime}$ and $(\psi \beta)^{*}$ respectively. Finally, let $\rho$ be any simplex of $\psi$ such that $b_{4}<\eta(\rho)$. Then:

(1) $\left|D^{*}(\rho, \psi \beta)\right|=\eta^{-1}(Q) \cap|D(\rho, \psi \beta)|$,

(2) $\left|C\left(D^{*}(\rho, \psi), D^{*}(\rho, \psi \beta)\right)\right|=\eta^{-1}\left(c_{3} b_{3}\right) \cap|D(\rho, \psi \beta)|$.

Proof. For simplicity we denote

$$
\begin{aligned}
C(\rho ; \psi, \psi \beta) & \equiv C(D(\rho, \psi), D(\rho, \psi \beta))=C(\dot{D}(\rho, \psi), \dot{D}(\rho, \psi \beta)), \\
C^{*}(\rho ; \psi, \psi \beta) & \equiv C\left(D^{*}(\rho, \psi), D^{*}(\rho, \psi \beta)\right)=C\left(\dot{D}^{*}(\rho, \psi), \dot{D}^{*}(\rho, \psi \beta)\right) .
\end{aligned}
$$

The proof is by induction on the codimension of $\rho$ in $\psi \beta$; i.e. on $n=(\operatorname{dim} \psi \beta-\operatorname{dim} \rho)$. The result is trivial when $n=0$. We assume that $n>0$ and the result is known for integers less than $n$. 
STEP I. Let $A=b\left(\rho_{0}\right) \cdots b\left(\rho_{s}\right) b^{*}\left(\rho_{s+1} \beta_{s+1}\right) \cdots b^{*}\left(\rho_{t} \beta_{t}\right)$ be a simplex of $D^{*}(\rho, \psi \beta)$ where $\rho<\rho_{0}<\cdots<\rho_{t}<\psi$, and $\varnothing \neq \beta_{s+1}<\cdots<\beta_{t}<\beta$. Recall that

$$
b^{*}\left(\rho_{j} \beta_{j}\right)=\left[\eta^{-1}\left(c_{3} b_{3}\right)\right] \cap\left[b\left(\rho_{j}\right) b\left(\rho_{j} \beta_{j}\right)\right] .
$$

Thus $\eta$ takes each vertex of $A$ into $Q$. Since $Q$ is convex and $\eta$ is linear on $\psi \beta$ it follows that $\eta(A) \subset Q$. Also it is clear that each vertex of $A$ belongs to $|D(\rho, \psi \beta)|$. The latter set is convex by (6.2) so $A \subset \eta^{-1}(Q) \cap|D(\rho, \psi \beta)|$. In case $s=-1$, i.e. $A<C^{*}(\rho ; \psi, \psi \beta)$, this argument shows that $A \subset \eta^{-1}\left(c_{3} b_{3}\right) \cap|D(\rho, \psi \beta)|$. Hence $\left|D^{*}(\rho, \psi \beta)\right| \subset \eta^{-1}(Q) \cap|D(\rho, \psi \beta)|$ and $C^{*}(\rho ; \psi, \psi \beta) \subset \eta^{-1}\left(c_{3} b_{3}\right) \cap|D(\rho, \psi \beta)|$.

STEP II. We claim that

(i) $\eta^{-1}(Q) \cap D(\rho, \psi \beta)$ is an $n$-ball,

(ii) $\eta^{-1}\left(c_{3} b_{3}\right) \cap D(\rho, \psi \beta)$ is an $(n-1)$-ball,

(iii) $\partial\left[\left(\eta^{-1} Q\right) \cap D(\rho, \psi \beta)\right]=\left[\left(\eta^{-1} Q\right) \cap \partial D(\rho, \psi \beta)\right] \cup\left[\left(\eta^{-1}\left(c_{3} b_{3}\right)\right) \cap D(\rho, \psi \beta)\right]$,

(iv) $\partial\left[\left(\eta^{-1}\left(c_{3} b_{3}\right)\right) \cap D(\rho, \psi \beta)\right]=\left(\eta^{-1}\left(c_{3} b_{3}\right)\right) \cap \partial D(\rho, \psi \beta)$.

To see this, notice that there is a first derived $Q_{0}^{\prime}$ of $Q_{0}$ such that

$$
Q=\left|N\left(\left(c_{4} b_{4}\right)^{\prime}, Q_{0}^{\prime}\right)\right| \quad \text { and } \quad c_{3} b_{3}=\left|\dot{N}\left(\left(c_{4} b_{4}\right)^{\prime}, Q_{0}^{\prime}\right)\right| \text {. }
$$

If we take a first derived of $D(\rho, \psi \beta)$ such that $\eta \mid D(\rho, \psi \beta)^{\prime}: D(\rho, \psi \beta)^{\prime} \rightarrow Q_{0}^{\prime}$ is simplicial then it follows from (2.14) that

$$
\begin{aligned}
\left(\eta^{-1} Q\right) & \cap D(\rho, \psi \beta)=N\left(D(\rho, \psi)^{\prime}, D(\rho, \psi \beta)^{\prime}\right), \\
\left(\eta^{-1}\left(c_{3} b_{3}\right)\right) & \cap D(\rho, \psi \beta)=\dot{N}\left(D(\rho, \psi)^{\prime}, D(\rho, \psi \beta)^{\prime}\right) .
\end{aligned}
$$

Then assertions (i)-(iv) are immediate consequences of the convexity of the sets in (i) and (ii) and the basic facts about first derived neighborhoods in a manifold (e.g. (3.4) of [C]).

STEP III. Clearly $D(\rho, \psi \beta)$ is an $n$-ball. We prove that $C(\rho ; \psi, \psi \beta)$ is an $(n-1)$ ball and we calculate $\partial D(\rho, \psi \beta)$ and $\partial C(\rho ; \psi, \psi \beta)$ explicitly. Analogous formulas hold for $D^{*}(\rho, \psi \beta)$ and $C^{*}(\rho ; \psi, \psi \beta)$. Let $\alpha=\operatorname{Lk}(\rho, \psi)$. Then

$$
\begin{aligned}
D(\rho, \psi \beta)= & \left\{b\left(\rho \alpha_{0} \beta_{0}\right) \cdots b\left(\rho \alpha_{q} \beta_{q}\right) \mid \alpha_{i}<\alpha, \beta_{i}<\beta\right\} \\
\partial D(\rho, \psi \beta)= & \dot{D}(\rho, \psi \beta) \cup D(\rho, \partial(\psi \beta)) \text { by }(5.6) \text { of }[C] \\
= & \left\{b\left(\rho \alpha_{0} \beta_{0}\right) \cdots b\left(\rho \alpha_{q} \beta_{q}\right) \mid\left(\beta_{0} \neq \varnothing \text { or } \alpha_{0} \neq \varnothing\right) \text { or }\left(\alpha_{q} \neq \alpha \text { or } \beta_{q} \neq \beta\right)\right\} \\
= & C(\rho ; \psi, \psi \beta) \cup(\bigcup\{D(\mu, \psi \beta) \mid \rho \varsubsetneqq \mu<\psi\}) \\
& \cup(\bigcup\{D(\rho, \bar{\psi} \beta) \mid \rho<\bar{\psi} \varsubsetneqq \psi\}) \cup\left(\bigcup\left\{D\left(\rho, \psi \beta_{q}\right) \mid \beta_{q} \varsubsetneqq \beta\right\}\right) .
\end{aligned}
$$

Under the natural isomorphism between $\dot{D}(\rho, \psi \beta)$ and $\operatorname{Lk}(\rho, \psi \beta)^{\prime}=(\alpha \beta)^{\prime}$, $C(\rho ; \psi, \psi \beta)$ goes onto $N\left(\beta^{\prime},(\alpha \beta)^{\prime}\right)$. This proves that $C(\rho ; \psi, \psi \beta)$ is an $(n-1)$-ball. Using the fact that $\partial N\left(\beta^{\prime},(\alpha \beta)^{\prime}\right)=\dot{N}\left(\beta^{\prime},(\alpha \beta)^{\prime}\right) \cup N\left(\beta^{\prime}, \partial(\alpha \beta)^{\prime}\right)$ we can calculate $\partial N\left(\beta^{\prime},(\alpha \beta)^{\prime}\right)$ just as we calculated $\partial D(\rho, \psi \beta)$ above. Returning under the inverse isomorphism this yields:

$$
\begin{aligned}
\partial C(\rho ; \psi, \psi \beta)= & (\bigcup\{C(\mu ; \psi, \psi \beta) \mid \rho \supsetneqq \mu<\psi\}) \\
& \cup(\bigcup\{C(\rho ; \bar{\psi}, \bar{\psi} \beta) \mid \rho<\bar{\psi} \supsetneqq \psi\}) \cup\left(\bigcup\left\{C\left(\rho ; \psi, \psi \beta_{q}\right) \mid \beta_{q} \supsetneqq \beta\right\}\right) .
\end{aligned}
$$


STEP IV. We prove that $\left|C^{*}(\rho ; \psi, \psi \beta)\right|=\left(\eta^{-1}\left(c_{3} b_{3}\right)\right) \cap|D(\rho, \psi \beta)|$. We have already shown (Step I) that one of these is included in the other and we know (Steps II and III) that each is an (n-1)-ball. Thus it suffices to prove that

$$
\left|\partial C^{*}(\rho ; \psi, \psi \beta)\right|=\partial\left[\eta^{-1}\left(c_{3} b_{3}\right) \cap|D(\rho, \psi \beta)|\right]
$$

But

$$
\begin{array}{r}
\partial C^{*}(\rho ; \psi, \psi \beta)=\left(\bigcup\left\{C^{*}(\mu ; \psi, \psi \beta) \mid \rho \supsetneqq \mu<\psi\right\}\right) \cup\left(\bigcup\left\{C^{*}(\rho ; \bar{\psi}, \bar{\psi} \beta) \mid \rho<\bar{\psi} \supsetneqq \psi\right\}\right) \\
\cup\left(\bigcup\left\{C^{*}\left(\rho ; \psi, \psi \beta_{q}\right) \mid \beta_{q} \supsetneqq \beta\right\}\right), \quad \text { by Step III, } \\
=\eta^{-1}\left(c_{3} b_{3}\right) \cap[\bigcup\{D(\mu, \psi \beta) \mid \rho ł \mu<\psi\} \cup(\bigcup\{D(\rho, \bar{\psi} \beta) \mid \rho<\bar{\psi} \supsetneqq \psi\}) \\
\left.\cup\left(\bigcup\left\{D\left(\rho, \psi \beta_{q}\right) \mid \beta_{q} \supsetneqq \beta\right\}\right) \cup C(\rho ; \psi, \psi \beta)\right],
\end{array}
$$

noting that $\left(\eta^{-1}\left(c_{3} b_{3}\right)\right) \cap C(\rho ; \psi, \psi \beta)=0$, and using the induction hypothesis

$$
\begin{aligned}
& =\eta^{-1}\left(c_{3} b_{3}\right) \cap \partial D(\rho, \psi \beta) \quad \text { by Step III } \\
& =\partial\left[\eta^{-1}\left(c_{3} b_{3}\right) \cap D(\rho, \psi \beta)\right] \quad \text { by (iv) of Step II. }
\end{aligned}
$$

This proves assertion (2) of our lemma.

STEP V. To prove that $\left|D^{*}(\rho, \psi \beta)\right|=\eta^{-1} Q \cap|D(\rho, \psi \beta)|$ we note that these are each $n$-balls and that one is included in the other. Thus it suffices to prove that their boundaries coincide. The argument is similar to Step IV, q.e.d.

Proof of (6.1c). We shall be working with the simplicial complex $J$. To simplify the notation we write $V=N(K-L, J), \dot{V}=\dot{N}(K-L, J)$ and $W=C(K-L, J)$. We may assume (taking a barycentric subdivision of $J$ modulo $V$ if necessary) that $V$ and $\dot{V}$ are full in $J$.

Let $\Delta=a b_{0} b_{4}$. (See Figure 1 and (6.3).) Let $\Delta^{\prime}$ be the barycentric subdivision of $\Delta$ and let $\Delta^{*}$ be the first derived with vertices $a, b_{0}, b_{4}, c_{0}, b_{3}, c_{4}$, and $c_{3}$. As usual $\eta: N(K-L, J) \rightarrow \Delta$ is the natural parametrization. Let $J^{\prime}$ be a first derived of $J$ such that $b(\sigma)$ is the centroid of $(\eta \mid \sigma)^{-1} b(\eta(\sigma))$, for each simplex $\sigma<N(K-L, J)$. Define $J^{*}$ as the first derived of $J$ which agrees with $J^{\prime}$ on $C(K-L, J) \cup K$ and for which $b^{*}(\rho \beta)=\eta^{-1}\left(c_{3} b_{3}\right) \cap b(\rho) b(\rho \beta)$ whenever $\rho<(\dot{V}-L), \quad \varnothing \neq \beta<C(L, K)$ and $\rho \beta<J$. Notice that $\eta: J^{\prime} \rightarrow \Delta^{\prime}$ and $\eta: J^{*} \rightarrow \Delta^{*}$ are simplicial mappings. Simplicial structures relating to $J^{\prime}$ are denoted as usual, while those relating to $J^{*}$ are denoted by asterisks. If $\rho<S<T<J$ then $C(\rho ; S, T) \equiv C(D(\rho, S), D(\rho, T))$ and $C^{*}(\rho ; S, T)$ $\equiv C\left(D^{*}(\rho, S), D^{*}(\rho, T)\right)$. The symbol $\rho$ always denotes a variable nonempty simplex of $\dot{V}-L$. Finally, we set

$$
\begin{array}{rlrl}
\mathscr{N} & =N\left(\dot{V}-L, J^{\prime}\right), & & \dot{\mathscr{N}}=\dot{N}\left(\dot{V}-L, J^{\prime}\right), \\
\mathscr{N}_{V}=N\left(\dot{V}-L, V^{\prime}\right), & \dot{\mathscr{N}}_{V}=\dot{N}\left(\dot{V}-L, V^{\prime}\right), \\
\mathscr{N}_{V}^{*}=N\left(\dot{V}-L, V^{*}\right), & \dot{\mathscr{N}}_{V}^{*}=\dot{N}\left(\dot{V}-L, V^{*}\right) .
\end{array}
$$

(See Figure 2.) 


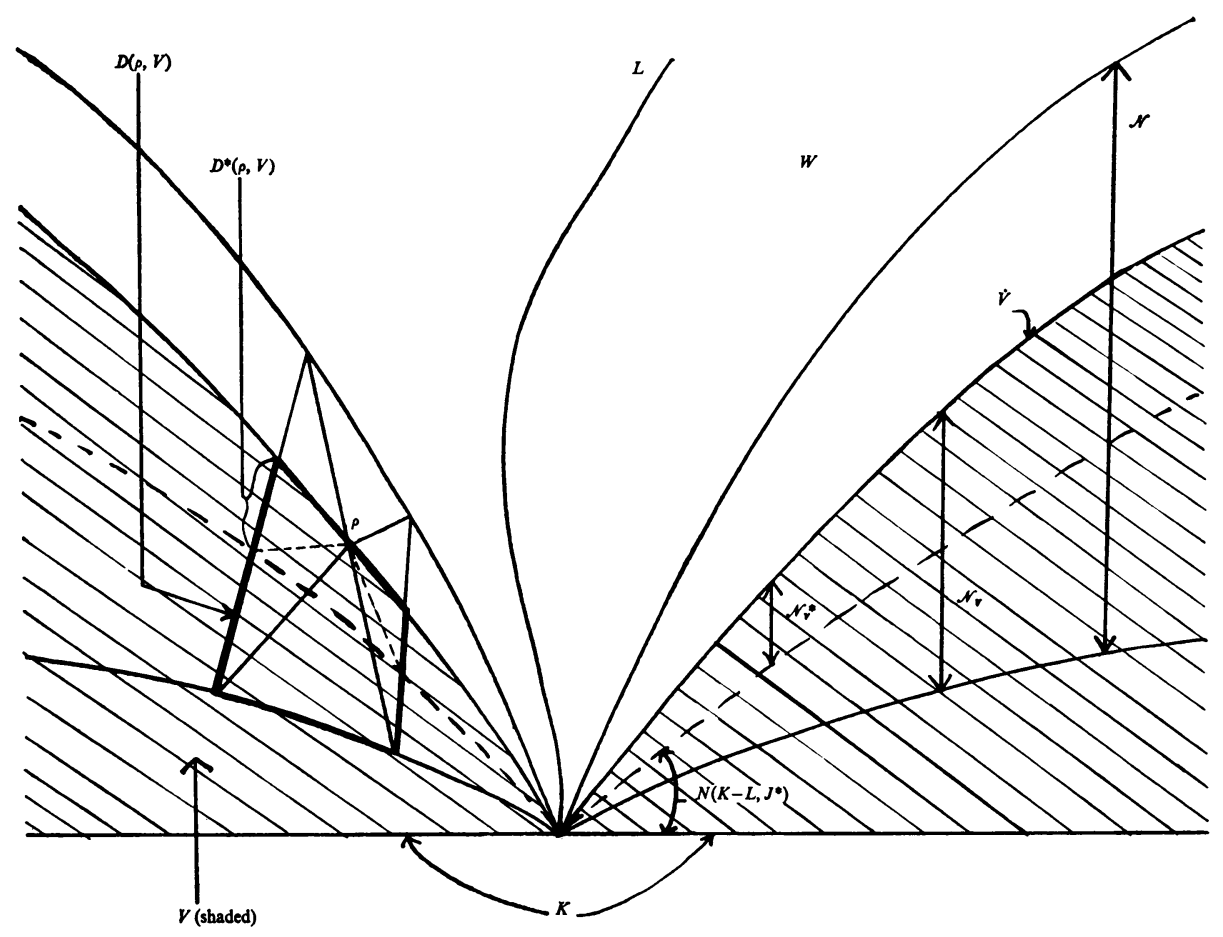

FIGURE 2

STEP I. We apply the theorem on collared subpolyhedra (4.2D) to the triples $(V, \dot{V}, V \cap L)$ and $(W, \dot{V}, L)$. Thus there is a homeomorphism $g: C\left(L^{\prime}, \mathcal{N}\right)$ $\rightarrow C\left(L^{\prime}, \dot{V}^{\prime}\right) \times[0,3]$ such that, for each simplex $\rho<\dot{V}-L$,

$$
\begin{gathered}
D(\rho, W) \rightarrow D(\rho, \dot{V}) \times[2,3], \\
D(\rho, V) \rightarrow D(\rho, \dot{V}) \times[0,2], \\
D(\rho, \dot{V}) \stackrel{1}{\rightarrow} D(\rho, \dot{V}) \times 2, \\
C(\rho ; \dot{V}, W) \rightarrow D(\rho, \dot{V}) \times 3, \\
C(\rho ; \dot{V}, V) \rightarrow D(\rho, \dot{V}) \times 0 .
\end{gathered}
$$

Claim. $g$ can be chosen so as to further satisfy

$$
\begin{aligned}
C\left(L^{\prime}, \mathscr{N}_{V}^{*}\right) & \rightarrow C\left(L^{\prime}, \dot{V}^{\prime}\right) \times[1,2], \\
D^{*}(\rho, V) & \rightarrow D(\rho, \dot{V}) \times[1,2], \\
C^{*}(\rho ; \dot{V}, V) & \rightarrow D(\rho, \dot{V}) \times 1 .
\end{aligned}
$$


LEMMA USED TO PROVE THE CLAIM:

(1) $\eta^{-1}(Q)=C\left(L^{\prime}, \mathscr{N}_{V}^{*}\right)$ and $\eta^{-1}\left(c_{3} b_{3}\right)=C\left(L^{\prime}, \dot{\mathscr{N}}_{V}^{*}\right)$.

(2) If $\rho<\dot{V}-L$ then

$$
\eta^{-1}(Q) \cap D(\rho, V)=D^{*}(\rho, V) \text { and } \eta^{-1}\left(c_{3} b_{3}\right) \cap D(\rho, V)=C^{*}(\rho ; \dot{V}, V) .
$$

(3) If $\rho<\psi<\dot{V}-L, \rho<\dot{V}-L, \psi \beta<V$, and $\beta<C(L, K)$, then

$$
\eta^{-1}(Q) \cap D(\rho, \psi \beta)=D^{*}(\rho, \psi \beta) \text { and } \eta^{-1}\left(c_{3} b_{3}\right) \cap D(\rho, \psi \beta)=C^{*}(\rho ; \psi, \psi \beta) .
$$

Proof of the lemma. By taking successive unions one proves easily that (3) $\Rightarrow(2) \Rightarrow(1)$. But (3) is precisely the conclusion of (6.3).

Proof of the claim. $\eta \mid C\left(L^{\prime}, \mathscr{N}_{v}\right)$ is a simplicial mapping into $Q_{0}$. There is a first derived $Q_{0}^{\prime}$ of $Q_{0}$ such that

$$
Q=\left|N\left(c_{4} b_{4}-c_{2} b_{2}, Q_{0}^{\prime}\right)\right| \quad \text { and } \quad c_{3} b_{3}=\left|\dot{N}\left(c_{4} b_{4}-c_{2} b_{2}, Q_{0}^{\prime}\right)\right| \text {. }
$$

We choose a first derived $C\left(L^{\prime}, \mathscr{N}_{V}\right)^{\prime}$ such that $\eta: C\left(L^{\prime}, \mathscr{N}_{V}\right)^{\prime} \rightarrow Q_{0}^{\prime}$ is also simplicial. Then, by (2.14), $\left(\eta^{-1}(Q),\left\{\eta^{-1}(Q) \cap D(\rho, V)\right\}\right)$ is a regular neighborhood of $C\left(L^{\prime}, \dot{V}^{\prime}\right) \bmod C\left(L^{\prime}, \dot{\mathscr{N}}_{V}\right)$ in $\left(C\left(L^{\prime}, \mathscr{N}_{V}\right),\{D(\rho, V)\}\right)$. Thus by the lemma, $\left(C\left(L^{\prime}, \mathscr{N}_{V}^{*}\right),\left\{D^{*}(\rho, V)\right\}\right)$ is such a regular neighborhood. Notice also that the boundary of this regular neighborhood is $\left(C\left(L^{\prime}, \dot{\mathscr{N}}_{V}^{*}\right),\left\{C^{*}(\rho ; \dot{V}, V)\right\}\right)$.

From the above we see that $g\left(C\left(L^{\prime}, \mathscr{N}_{V}^{*}\right),\left\{D^{*}(\rho, V)\right\}\right)$ is a regular neighborhood of $C\left(L^{\prime}, \dot{V}^{\prime}\right) \times 2 \bmod C\left(L^{\prime}, \dot{V}^{\prime}\right) \times 0$ in $\left(C\left(L^{\prime}, \dot{V}^{\prime}\right) \times[0,2],\{D(\rho, \dot{V}) \times[0,2]\}\right)$. Therefore we may apply Addendum 3.3 of the uniqueness theorem to get a homeomorphism $g_{1}$ of $\left(C\left(L^{\prime}, \dot{V}^{\prime}\right) \times[0,2],\{D(\rho, \dot{V}) \times[0,2]\}\right)$ onto itself such that

$$
\begin{aligned}
g_{1} \mid C\left(L^{\prime}, \dot{V}^{\prime}\right) \times\{0,2\} & =1, \\
g_{1} g C\left(L^{\prime}, \mathscr{N}_{V}^{*}\right) & =C\left(L^{\prime}, \dot{V}^{\prime}\right) \times[1,2], \\
g_{1} g D^{*}(\rho, V) & =D(\rho, \dot{V}) \times[1,2], \\
g_{1} g C^{*}(\rho ; \dot{V}, V) & =D(\rho, \dot{V}) \times 1 .
\end{aligned}
$$

If we define $g_{1}$ to be the identity on $C\left(L^{\prime}, \dot{V}^{\prime}\right) \times[2,3]$ then $g_{1} g=g_{2}$ is a homeomorphism which satisfies the claim.

STEP II. We now define a homeomorphism $h_{0}$ of $J$ onto $J$ such that $h_{0} \mid C\left(\dot{V}-L, J^{\prime}\right)=1 \quad$ and $h_{0}(V)=N\left(K-L, J^{*}\right)$. Since $\left(K^{\prime} \cup L^{\prime}\right)<C\left(\dot{V}-L, J^{\prime}\right)$, $h_{0}$ is the identity on $\left(K^{\prime} \cup L^{\prime}\right)$. (For the rest of this paragraph let $V=h N(K-L, J)$.) Hence $\left(J, K, L ; h h_{0}^{-1}\right)$ is a full triangulation of $(Z, X, Y)$ such that

$$
V=h h_{0}^{-1} N\left(K-L, J^{*}\right) \text {. }
$$

This proves that $V$ is a regular neighborhood of $X \bmod Y$ in $Z$.

Let $u:[0,3] \rightarrow[0,3]$ be a homeomorphism such that $u(0)=0, u(2)=1$ and $u(3)=3$. Let $k=1 \times u: C\left(L^{\prime}, \dot{V}^{\prime}\right) \times[0,3] \rightarrow C\left(L^{\prime}, \dot{V}^{\prime}\right) \times[0,3]$. Let $g$ be the homeomorphism constructed in Step I. Then $g^{-1} \mathrm{~kg}=h_{1}$ is a homeomorphism of $C\left(L^{\prime}, \mathscr{N}\right)$ 
onto itself. Using the properties of $g$ we see that

(II.1) $h_{1} D(\rho, J)=D(\rho, J)$ for each $\rho<\dot{V}-L$,

(II.2) $h_{1} \mid C(\rho ; \dot{V}, J)=1$,

(II.3) $h_{1} D(\rho, \dot{V})=C^{*}(\rho ; \dot{V}, V)$,

(II.4) $h_{1} D(\rho, W)=D^{*}(\rho, J)$.

Let $L_{0}=L \cap N(\dot{V}-L, \dot{V})$. Then, by (2.10),

$$
\begin{aligned}
\mathscr{N} & =N\left(\dot{V}-L, J^{\prime}\right) \\
& =\left\{b\left(\alpha_{0} \gamma_{0} \delta_{0}\right) \cdots b\left(\alpha_{q} \gamma_{q} \delta_{q}\right) \mid \alpha_{i} \gamma_{i} \delta_{i}<N(\dot{V}-L, J), \gamma_{i}=\varnothing \Rightarrow \delta_{i}=\varnothing\right\} \\
& =\bigcup\{A D(\rho, J) \mid \rho<(\dot{V}-L) \text { and : } \\
& \left.A=\varnothing \text { or } A=b\left(\alpha_{0}\right) \cdots b\left(\alpha_{r}\right)<L_{0}^{\prime} \text { with } \alpha_{r}<\rho\right\} \\
\dot{\mathscr{N}}= & \bigcup\{A C(\rho ; \dot{V}, J) \mid \rho<(\dot{V}-L) \text { and: } \\
\left.\qquad A=\varnothing \text { or } A=b\left(\alpha_{0}\right) \cdots b\left(\alpha_{r}\right)<L_{0}^{\prime} \text { with } \alpha_{r}<\rho\right\} & \\
C\left(L^{\prime}, \mathscr{N}\right)=\bigcup\{D(\rho, J) \mid \rho<(\dot{V}-L)\} & \\
C\left(L^{\prime}, \dot{\mathscr{N}}\right) & =\bigcup\{C(\rho ; \dot{V}, J) \mid \rho<(\dot{V}-L)\} .
\end{aligned}
$$

Extend $h_{1}$ to a mapping $h_{2}: \mathscr{N} \rightarrow \mathscr{N}$ by stipulating that

$$
h_{2}\left|[A * D(\rho, J)]=1_{A} * h_{1}\right| D(\rho, J) .
$$

By (II.1), $h_{2} \mid A D(\rho, J)$ is a homeomorphism of $A D(\rho, J)$ onto itself. Also

$$
\left(A_{0} D\left(\rho_{0}, J\right)\right) \cap\left(A_{1} D\left(\rho_{1}, J\right)\right)=\left(A_{0} \cap A_{1}\right) D\left(\rho_{0} \cdot \rho_{1}, J\right),
$$

where $\rho_{0} \cdot \rho_{1}$ is the smallest simplex of $J$ containing both $\rho_{0}$ and $\rho_{1}$, and this dual is understood to be empty if there is no such simplex. Therefore $h_{2}$ is a well-defined homeomorphism of $\mathscr{N}$ with itself. From (II.2) it is clear that

$$
h_{2}\left|\dot{\mathscr{N}}=h_{2}\right| \dot{N}\left(\dot{V}-L, J^{\prime}\right)=1 .
$$

So $h_{2}$ extends to a homeomorphism $h_{0}$ of $J$ which is the identity on $C\left(\dot{V}-L, J^{\prime}\right)$. It remains only to show that $h_{0}(V)=N\left(K-L, J^{*}\right)$.

Suppose that $x \in J-V$. If $h_{0}(x)=x$ then $h_{0}(x) \notin N\left(K-L, J^{*}\right)$. If $h_{0}(x) \neq x$ then $x \in[A D(\rho, W)-A D(\rho, \dot{V})]$ for some simplexes $A<L_{0}$ and $\varnothing \neq \rho<\dot{V}-L$. Therefore, by (II.3) and (II.4), $h_{0}(x) \in\left[A D^{*}(\rho, J)-A C^{*}(\rho ; \dot{V}, V)\right]$. Hence the carrier of $h_{0}(x)$ in $J^{*}$ is of the form $B=b\left(\alpha_{0}\right) \cdots b\left(\alpha_{q}\right) b^{*}\left(\rho_{0}\right) b^{*}\left(\rho_{1} \beta_{1}\right) \cdots b^{*}\left(\rho_{r} \beta_{r}\right)$, where $\rho<\rho_{0}$ $<\dot{V}-L$. Thus $B \nless N\left(K-L, J^{*}\right)$, and we see that, in any case, $x \notin V$ implies $h_{0}(x) \notin N\left(K-L, J^{*}\right)$. Therefore $N\left(K-L, J^{*}\right)<h_{0}(V)$.

To prove the opposite inclusion notice that, on the one hand, $h_{0} N\left(K-L, J^{\prime}\right)$ $=N\left(K-L, J^{\prime}\right)=\eta^{-1}\left(a b_{0} b_{2}\right)<\eta^{-1}\left(a b_{0} b_{3}\right)=N\left(K-L, J^{*}\right)$. On the other hand, if $B$ is a simplex of $N(K-L, J)^{\prime}-N\left(K-L, J^{\prime}\right)$ then

$$
\boldsymbol{B}=b\left(\alpha_{0}\right) \cdots b\left(\alpha_{q}\right) b\left(\alpha_{q+1} \gamma_{q+1}\right) b\left(\alpha_{q+1} \beta_{q+2} \gamma_{q+2}\right) \cdots b\left(\alpha_{r} \beta_{r} \gamma_{r}\right)
$$

where $\alpha_{i} \beta_{i} \gamma_{i}<N(K-L, J)$ and $\varnothing \neq \gamma_{q+1}<\dot{N}\left(K_{R}, N(K-L, J)\right)$. Let $\rho=\alpha_{q+1} \gamma_{q+1}$. 
Then $\rho<(\dot{V}-L)$ because $V \cap L=L_{R}$. Thus $B<A D(\rho, V)$ where $A<L_{0}^{\prime}$ and $\rho<\dot{V}-L$. Hence

$$
\begin{aligned}
h_{0}(B) & <A * h_{0} D(\rho, V) \\
& =A * h_{0}(\mathrm{Cl}[D(\rho, J)-D(\rho, W)]) \\
& =A * \mathrm{Cl}\left[D(\rho, J)-D^{*}(\rho, J)\right], \quad \text { by (II.1), (II.4). }
\end{aligned}
$$

Let $P$ denote the closure in the last expression. Now $D^{*}(\rho, J)>D(\rho, J) \cap \eta^{-1} Q$ by the lemma in Step I; so if $x \in P$ then $\eta(x) \in a b_{0} b_{3}$. Since $A<L_{R}^{\prime}, \eta(A)=a$. Hence, by linearity of $\eta, \eta(A * P)<a b_{0} b_{3}$. Thus

$$
h_{0}(B)<A * P<\eta^{-1}\left(a b_{0} b_{3}\right)=N\left(K-L, J^{*}\right) .
$$

Therefore $h_{0}(V)=N\left(K-L, J^{*}\right)$. The proof of (6.1c) is complete, q.e.d.

Proof of (6.1b). We assume that $\left(\mathrm{Bdy}_{z} V\right)-Y$ is locally bicollared in $Z$ and that there is a stellar triangulation $(J, K, L ; h)$ of $\left(V, X_{R}, Y_{R}\right)$ with $h \dot{N}(K-L, J)$ $=\left(\operatorname{Bdy}_{z} V\right) \cup Y_{R}$.

Since

$$
\left(\operatorname{Bdy}_{z} V\right) \cup Y_{R}<\mathrm{Cl}((Z-V)) \cup Y_{R},
$$

it follows from the definition of a subpolyhedron that there is a partition $(\dot{N}(K-L, J))_{*}$ and a triangulation

of

$$
\left(Q, \dot{N}(K-L, J)_{*}, K_{0}, L_{0} ; q\right)
$$

$\left(\mathrm{Cl}((Z-V)) \cup Y_{R},\left(\mathrm{Bdy}_{z} V\right) \cup Y_{R}, \mathrm{Cl}((X-V)) \cup Y_{R}, \mathrm{Cl}((Y-V)) \cup Y_{R}=Y\right)$

such that

and

$$
q|| \dot{N}(K-L, J)_{*}|=h||\dot{N}(K-L, J)|
$$

$$
|Q| \cap|J|=q^{-1}\left(\left(\operatorname{Bdy}_{z} V\right) \cup Y_{R}\right)=|\dot{N}(K-L, J)| \text {. }
$$

We may assume that each of the subcomplexes mentioned is full in $Q$.

Extend the partition $\dot{N}(K-L, J)_{*}$ to a partition $J_{*}$ of $J=N(K-L, J)$ by demanding that $(\alpha \beta \gamma)_{*}=\beta\left((\alpha \gamma)_{*}\right)$ for all $\alpha \beta \gamma<J$. This gives a well-defined triangulation of $\left|J_{*}\right| \cup|Q|$ because $\left|J_{*}\right| \cap|Q|$ inherits the same triangulation from $J_{*}$ and from $Q$. Let $J_{1}=J_{*} \cup Q, K_{1}=K_{*} \cup K_{0}$ and $L_{1}=L_{0}$. Define $h_{1}: J_{1} \rightarrow Z$ by the condition that $h_{1} \mid J_{*}=h$ and $h_{1} \mid Q=q$. One checks easily that $\left(J_{1}, K_{1}, L_{1} ; h_{1}\right)$ is a full triangulation of $(Z, X, Y)$. Also, notice that $N(K-L, J)_{*}=N\left(K_{1}-L_{1}, J_{1}\right)$ and $\dot{N}(K-L, J)_{*}=\dot{N}\left(K_{1}-L_{1}, J_{1}\right)$. Therefore $V=h_{1} N\left(K_{1}-L_{1}, J_{1}\right)$. Finally,

$$
h_{1}\left(\dot{N}\left(K_{1}-L_{1}, J_{1}\right)-L_{1}\right)=h(\dot{N}(K-L, J)-L)=\left(\operatorname{Bdy}_{z} V\right)-Y \text {. }
$$

Hence $\dot{N}\left(K_{1}-L_{1}, J_{1}\right)-L_{1}$ is locally bicollared in $J_{1}$. So, since this set lies in the topological boundary of $N\left(K_{1}-L_{1}, J_{1}\right)-L_{1}$, it is locally collared in $N\left(K_{1}-L_{1}, J_{1}\right)$ and in $C\left(K_{1}-L_{1}, J_{1}\right)$. The hypotheses of (6.1c) are satisfied. Therefore $V$ is a regular neighborhood of $X \bmod Y$ in $Z$, q.e.d. 
Proof of (6.1a). We are given a stellar triangulation $(J, K, L ; h)$ of $\left(V, X_{R}, Y_{R}\right)$ such that $h(\dot{N}(K-L, J)-L)$ is locally collared in $V$. Let $J^{\prime}$ and $J^{*}$ be first deriveds of $J$ chosen as in the proof of (6.1c). Let $V_{1}=N\left(K-L, J^{*}\right)$ and $V_{0}=N\left(K-L, J^{\prime}\right)$. Clearly $V_{1}$ is a regular neighborhood of $X_{R} \bmod Y_{R}$ in $V$. The argument for (6.1c), restricted to $N(K-L, J)$, can be easily modified to yield an isotopy $G$ of $V$ into $V$ such that $G_{0}=1, G_{t} \mid V_{0}=1$ and $G_{1}(V)=V_{1}$. Some subdivision $\left(J_{0}, K_{0}, L_{0} ; h\right)$ of $\left(J^{\prime}, K^{\prime}, L^{\prime} ; h\right)$ extends to a full triangulation $\left(J_{1}, K_{1}, L_{1} ; h_{1}\right)$ of $(Z, X, Y)$. Let $U=h_{1} N\left(K_{1}-L_{1}, J_{1}^{\prime}\right)$ and let $U_{0}=h_{1} N\left(K_{1}-L_{1}, J_{1}^{\prime \prime}\right)$. These are both regular neighborhoods of $X \bmod Y$ in $Z$ and of $X_{R} \bmod Y_{R}$ in $V$. Then, by Addendum (3.2) (which will be proved in the next section, using (6.1c) and (6.1b)) there is an isotopy $F$ of $V$ onto itself such that $F_{0}=1, F_{t} \mid U_{0}=1$ and $F_{1}\left(V_{1}\right)=U$. Thus $H=F G$ is an isotopy of $V$ into $V$ with the desired properties, q.e.d.

7. Relationships between regular neighborhoods. Suppose that $X, Y, Z_{0}<Z$ and $X_{0}, Y_{0}<Z_{0}$. In this section we answer the following practical questions:

What is the effect on a regular neighborhood of increasing or decreasing the polyhedron $Y$ ?

What are the relationships between two regular neighborhoods of $X \bmod Y$ in $Z$ when one contains the other?

When can a regular neighborhood $V_{0}$, of $X \cap Z_{0} \bmod Y \cap Z_{0}$ in $Z_{0}$, be extended to a regular neighborhood $\left(V, V_{0}\right)$ of $X \bmod Y$ in $\left(Z, Z_{0}\right)$ ?

What is the relationship between $\left(V, V_{0}\right)$ being a regular neighborhood of $X$ mod $Y$ in $\left(Z, Z_{0}\right)$ and $V$ being a regular neighborhood of

in $Z$ ?

$$
\left(X \cup V_{0}\right) \bmod \left(Y \cup \mathrm{Bdy}_{z_{0}} V_{0}\right)
$$

Given two regular neighborhoods of $X_{0} \bmod Y_{0}$ in $Z_{0}$, when can an isotopy of $\left(Z, Z_{0}\right)$ be found which takes one onto the other and keeps fixed a crucial subpolyhedron of $Z$ ?

Proposition 7.1.

(a) If $V$ is a regular neighborhood of $X \bmod Y$ in $Z, P<Z$, and $(P \cap V)<Y$ then $V$ is a regular neighborhood of $X \bmod (Y \cup P)$ in $Z$.

(b) If $V$ is a regular neighborhood of $X \bmod (Y \cup P)$ in $Z$ and if $(P \cap X)<Y$ then $V$ is a regular neighborhood of $X \bmod Y$ in $Z$.

Proof. In both (a) and (b)

and

$$
\begin{aligned}
(X-Y) & =X-(Y \cup P), \\
Y_{R}=(Y \cup P)_{R} & =(V \cap Y)=V \cap(Y \cup P),
\end{aligned}
$$

$$
\left(\operatorname{Bdy}_{z} V\right)-Y=\left(\operatorname{Bdy}_{z} V\right)-(Y \cup P) .
$$

The result then follows in each case because the hypotheses in the stellar neighborhood theorem (6.1b) do not depend on what is happening outside $V$, q.e.d. 
The analogue of (7.1) for pairs-i.e., for regular neighborhoods $\left(V, V_{1}\right)$ of $X$ mod $Y$ (or of $X$ mod $Y \cup P)$ in $\left(Z, Z_{1}\right)$ - would follow from the stellar neighborhood theorem for pairs. The latter theorem appears to be true by a proof completely analogous to that of (6.1). Since however we have not developed the theory for pairs explicitly, we give a direct proof of that special case of (7.1) for pairs which will be needed in the sequel.

LEMMA 7.2. Suppose that $K, L \triangleleft J_{0} \triangleleft J$ and that $P$ is a subpolyhedron of $|J|$ such that $P \cap|N(K-L, J)|<|L|$. Then $\left(N\left(K-L, J^{\prime}\right), N\left(K-L, J_{0}^{\prime}\right)\right)$ is a regular neighborhood of $K \bmod (L \cup P)$ in $\left(J, J_{0}\right)$.

Proof. There is a partition $J_{*}$ of $J$ such that $|L| \cup P$ is the underlying space of a full subcomplex, say $Q$, of $J_{*}$. By definition the pair $\left(N\left(K-Q, J_{*}^{\prime}\right), N\left(K-Q, J_{0^{*}}^{\prime}\right)\right)$ is a regular neighborhood of $K \bmod (L \cup P)$ in $\left(J, J_{0}\right)$. But this pair is just

$$
\left(N\left(K-L, J_{*}^{\prime}\right), N\left(K-L, J_{0^{*}}^{\prime}\right)\right)
$$

since $P \cap|N(K-L, J)|<|L|$. By the proof of the uniqueness theorem, there is a homeomorphism fixed on $[K \cup C(K-L, J)]$ which takes this pair onto $\left(N\left(K-L, J^{\prime}\right)\right.$, $\left.N\left(K-L, J_{0}^{\prime}\right)\right)$. The prescribed homeomorphism obviously is fixed on $K \cup L \cup P$. Therefore $\left(N\left(K-L, J^{\prime}\right), N\left(K-L, J_{0}^{\prime}\right)\right)$ is a regular neighborhood of $K \bmod (L \cup P)$ in $\left(J, J_{0}\right)$, q.e.d.

Proposition 7.3. If $V$ and $V_{0}$ are regular neighborhoods of $X \bmod Y$ in $Z$ and $\left(V_{0}-Y\right) \subset \operatorname{Int}_{z} V$ then

(a) $V$ is a regular neighborhood of $V_{0} \bmod Y$ in $Z$;

(b) $\mathrm{Cl}(V-X)$ is a regular neighborhood of $V_{0} \bmod (X \cup Y)$ in $Z$.

Proof. To prove (a), let $(J, K, L ; h)$ be a full triangulation of $(Z, X, Y)$ exhibiting $V$ as a regular neighborhood, $V=h N\left(K-L, J^{\prime}\right)$. Let $V_{1}=h N\left(K-L, J^{\prime \prime}\right)$. The stellar neighborhood theorem (6.1b) implies that $V$ is a regular neighborhood of $V_{1} \bmod Y$ in $Z$. But (7.1a) implies that $V_{0}$ and $V_{1}$ are both regular neighborhoods of $X \bmod (\mathrm{Cl}(Z-V)) \cup Y$. Hence there is a homeomorphism $g$ of $Z$ onto itself such that $g(V)=V, g \mid Y=1$ and $g\left(V_{1}\right)=V_{0}$. Therefore $V$ is a regular neighborhood of $V_{0} \bmod Y$ in $Z$.

To prove (b), choose a full triangulation $\left(J, K_{0}, L ; h\right)$ of $\left(Z, V_{0}, Y\right)$ such that $h^{-1}(X)$ and $h^{-1}(X \cup Y)$ underlie full subcomplexes $K$ and $K \cup L$ of $J$. By part (a) there is a homeomorphism $g$ of $h N\left(K_{0}-L, J^{\prime}\right)$ onto $V$ such that $g \mid\left(V_{0} \cup Y\right)=1$. Thus $\left(J, K_{0}, K \cup L, g h\right)$ is a full triangulation of $\left(Z, V_{0}, X \cup Y\right)$ such that $g h\left(N\left(K_{0}-L, J^{\prime}\right)\right)=V$. Therefore

$$
g h\left(\mathrm{Cl}\left[N\left(K_{0}-L, J^{\prime}\right)-K\right]\right)=\mathrm{Cl}(V-X) .
$$

However, $\mathrm{Cl}\left[N\left(K_{0}-L, J^{\prime}\right)-K\right]=N\left(K_{0}-(K \cup L), J^{\prime}\right)$. Hence $\mathrm{Cl}(V-X)$ is a regular neighborhood of $V_{0} \bmod X \cup Y$ in $Z$, q.e.d. 
RemarK. Addendum (3.2) follows directly from (7.1), (7.3) and the uniqueness theorem for regular neighborhoods.

Proposition 7.4. If $V_{0}$ is a regular neighborhood of $X \bmod Y$ in $Z$ and $V$ is a regular neighborhood of $V_{0} \bmod Y$ in $Z$ then $V$ is a regular neighborhood of $X \bmod Y$ in $Z$.

Proof. Let $U$ be a regular neighborhood of $X \bmod Y$ in $Z$ such that $U-Y$ $\subset \operatorname{Int}_{z} V_{0}$. Since $(\mathrm{Cl}(Z-V)) \cap U<(\mathrm{Cl}(Z-V)) \cap V_{0}<Y$ it follows (3.2) that there is a homeomorphism $g: Z \rightarrow Z$ such that $g \mid Y=1, g(V)=V$ and $g\left(V_{0}\right)=U$. Hence the hypothesis implies that $V$ is a regular neighborhood of $U \bmod Y$ in $Z$. But, by (7.3), $V_{0}$ is a regular neighborhood of $U \bmod Y$ in $Z$. Therefore there is a homeomorphism $h$ of $Z$ onto itself such that $h\left(V_{0}\right)=V$ and $h \mid(U \cup Y)=1$. Then, since $V_{0}$ is a regular neighborhood of $X \bmod Y$ in $Z$, so is $V$, q.e.d.

Proposition 7.5. Suppose that $U$ and $V$ are regular neighborhoods of $X \bmod Y$ in $Z$ with $(U-Y) \subset \operatorname{Int}_{z} V$. Let $Y_{0}=Y \cap \mathrm{Bdy}_{z} V$. Then $\mathrm{Cl}(V-U)$ is homeomorphic to the regular neighborhood of $\left(\mathrm{Bdy}_{z} V\right) \times 0 \bmod \left(Y_{0} \times 0\right)$ in $\left(\mathrm{Bdy}_{z} V\right) \times I$.

Proof. Let $Z_{0}=V \cup\left(\mathrm{Bdy}_{z} V \times I\right)$ be the disjoint union with the identification $x=(x, 0)$ if $x \in \mathrm{Bdy}_{z} V$. It is obvious from the stellar neighborhood theorem $(6.1 \mathrm{~b})$ that $U$ and $V$ are regular neighborhoods of $X_{R} \bmod Y_{R}$ in $Z_{0}$. Let $W$ be a regular neighborhood of $V \bmod Y_{R}$ in $Z_{0}$. By checking a triangulation in which $W$ is a first derived neighborhood of $V$, one sees that $\mathrm{Cl}(W-V)$ is a regular neighborhood of $\mathrm{Bdy}_{z} V \bmod Y_{0}$ in $\left(\mathrm{Bdy}_{z} V\right) \times I$. Thus it suffices to exhibit a homeomorphism of $Z_{0}$ onto itself which takes $V$ onto $W$ and $U$ onto $V$.

By (7.3), $V$ is a regular neighborhood of $U \bmod Y_{R}$ in $Z_{0}$. Hence, by (7.4) $W$ is such a regular neighborhood. Therefore there is a homeomorphism $f$ of $Z_{0}$ onto itself such that $f \mid U=1$ and $f(V)=W$. Now $U$ and $V$ are regular neighborhoods of $X_{R} \bmod \left(Y_{R} \cup \mathrm{Cl}\left(Z_{0}-W\right)\right)$ in $Z_{0}$, by (7.1). Hence there is a homeomorphism $g$ of $Z_{0}$ onto itself such that $g(W)=W$ and $g(U)=V$. Then $g f(V, U)=g(W, U)=(W, V)$, q.e.d.

The following lemma is a special case of (7.9) and will be used to prove (7.9).

LEMMA 7.6. Suppose that $V_{0}$ is a regular neighborhood of $X \bmod Y$ in $Z_{0}$ where $X, Y<Z_{0}<Z$ and every triangulation of $Z_{0}$ extends to a triangulation of $Z$. Then, if $P<Z$ and $\left(P \cap Z_{0}\right)<Y$, there is a subpolyhedron $V$ of $Z$ such that $\left(V, V_{0}\right)$ is a regular neighborhood of $X \bmod (Y \cup P)$ in $\left(Z, Z_{0}\right)$.

REMARK. Every triangulation of $Z_{0}$ extends to one of $Z$ if, for example, $Z$ is a manifold and $Z_{0}=\partial Z$. Also (see [A] and [L]) every triangulation extends if $\left(Z, Z_{0}\right)$ is a proper locally unknotted manifold pair or $\left({ }^{3}\right)$ if $Z$ is a manifold and $Z_{0}$ is a compact, codimension 3 subpolyhedron in the interior of $Z$. (In the former case, C. P. Rourke has given an alternate proof, using block bundle theory, which

$\left({ }^{3}\right)$ The author is grateful to M. A. Armstrong for pointing this out. 
eliminates Armstrong's compactness assumption.) Most generally, E. C. Zeeman has asserted that every triangulation extends if and only if the intrinsic $i$-skeleton of $Z_{0}$ equals the ambient intrinsic $i$-skeleton of $Z_{0}$ in $Z$, for each integer $i$. However the proof of this assertion seems to be in doubt $\left({ }^{4}\right)$. [These comments are just background. We shall not use the results alluded to above.]

Proof. Choose a full triangulation $\left(J_{0}, K, L ; h_{0}\right)$ of $\left(Z_{0}, X, Y\right)$ such that $V_{0}$ $=h_{0} N\left(K-L, J_{0}^{\prime}\right)$. By hypothesis there is a triangulation $\left(J, J_{0}, h\right)$ of $\left(Z, Z_{0}\right)$ such that $h \mid J_{0}=h_{0}$. We may assume that $J_{0}$ is full in $J$. It would suffice to know that $h^{-1}(P) \cap N\left(J_{0}-L, J\right)<L$. For this implies that $h^{-1}(P) \cap N(K-L, J)<L$. Then, taking a first derived $J^{\prime}$ of $J$ which agrees on $J_{0}$ with $J_{0}^{\prime}$, we could apply (7.2) to conclude that $\left(N\left(K-L, J^{\prime}\right), N\left(K-L, J_{0}^{\prime}\right)\right)$ is a regular neighborhood of $K \bmod \left(L \cup h^{-1} P\right)$ in $\left(J, J_{0}\right)$. It would follow that the image $\left(V, V_{0}\right)$ of this pair under $h$ is a regular neighborhood of $X \bmod (Y \cup P)$ in $\left(Z, Z_{0}\right)$.

Unfortunately it might not occur that $h^{-1}(P) \cap N\left(J_{0}-L, J\right)<L$. However, let $\tilde{J}$ be a first barycentric subdivision of $J$ modulo $J_{0}$ and let $g: J \rightarrow J$ be a homeomorphism such that

(1) $g \mid J_{0}=1$,

(2) $h^{-1}(P) \cap g N\left(J_{0}-L, \tilde{J}\right)<L$.

Then $\left(\tilde{J}, J_{0} ; h g\right)$ is a triangulation of $\left(Z, Z_{0}\right)$ with all the desired properties. We shall show that such a homeomorphism $g$ exists.

Notice that $\left|N\left(J_{0}-L, \tilde{J}\right)\right|=\left|N\left(J_{0}-L, J^{\prime}\right)\right|$ and interpret (2) accordingly.

$g$ is defined to be the identity on $J_{0} \cup C\left(J_{0}-L, J\right)$. Suppose that $g$ has been extended inductively over

$$
J_{i}=J_{0} \cup C\left(J_{0}-L, J\right) \cup(i \text {-skeleton of } J)
$$

so that, for each $\sigma<J_{i}$ we have

(a) $g(\sigma)=\sigma$,

(b) $h^{-1}(P) \cap g N\left(\sigma \cap J_{0}-\sigma \cap L, \sigma^{\prime}\right)<L$.

We extend $g$ over each simplex $\tau<J_{i+1}-J_{i}$. Since $\tau<N\left(J_{0}-L, J\right)-J_{i}, \tau=\alpha \beta \gamma$ where $\alpha<L, \beta<C\left(L, J_{0}\right), \gamma<C\left(J_{0}, J\right), \beta \neq \varnothing$, and $\gamma \neq \varnothing$. By hypothesis

$$
\left[h^{-1}(P) \cap \tau \cap J_{0}\right]<|\alpha| \text {. }
$$

By inductive assumption

$$
h^{-1}(P) \cap g N\left(\dot{\tau} \cap J_{0}-\dot{\tau} \cap L, \dot{\tau}^{\prime}\right)=h^{-1}(P) \cap g N\left(\alpha \beta-\alpha, \dot{\tau}^{\prime}\right)<|\alpha| .
$$

Let $g_{\tau}$ be a homeomorphism of $\tau$ onto $\tau$, fixed on $\dot{\tau}$, which takes

$$
b(\tau) * g N\left(\alpha \beta-\alpha, \dot{\tau}^{\prime}\right)=\left(1_{b(\tau)} * g \mid \dot{\tau}\right)\left(N\left(\alpha \beta-\alpha, \tau^{\prime}\right)\right)
$$

onto a regular neighborhood $W$ of $g N\left(\alpha \beta-\alpha, \dot{\tau}^{\prime}\right) \bmod \left[h^{-1}(P) \cup g C\left(\alpha \beta-\alpha, \dot{\tau}^{\prime}\right)\right]$ in $\tau$. This is possible because, on the one hand, $b(\tau) * g N\left(\alpha \beta-\alpha, \dot{\tau}^{\prime}\right)$ is a regular

(4) A proof of this in the compact case has now appeared in E. Akin's thesis, Manifold phenomena in the theory of polyhedra, Princeton Univ., Princeton, N. J., 1968. 
neighborhood of $g N\left(\alpha \beta-\alpha, \dot{\tau}^{\prime}\right) \bmod g C\left(\alpha \beta-\alpha, \dot{\tau}^{\prime}\right)$ by the stellar neighborhood theorem. On the other hand $W$ is such a regular neighborhood because

$$
\left[h^{-1}(P) \cap g N\left(\alpha \beta-\alpha, \dot{\tau}^{\prime}\right)\right]<|\alpha|<g C\left(\alpha \beta-\alpha, \dot{\tau}^{\prime}\right)
$$

and we may apply (7.1). Notice that $g_{\tau} \mid \dot{\tau}=1$ and $W \cap h^{-1}(P)<|\alpha|$.

Now we extend $g$ over each $\tau<J_{i+1}-J_{i}$ by defining

$$
g \mid \tau=g_{\imath} \circ\left(1_{b(\alpha)} * g \mid \dot{\tau}\right) \text {. }
$$

Continuing in this way we define $g$ on all of $J$. It is easy to see that $g$ has properties (1) and (2), q.e.d.

Proposition 7.7. Suppose that $V_{0}$ and $W_{0}$ are regular neighborhoods of $X \bmod Y$ in $Z_{0}$, where $X, Y<Z_{0}<Z$ and every triangulation of $Z_{0}$ extends to a triangulation of $Z$. Then, if $P<Z$ and $\left(P \cap Z_{0}\right)<(X \cup Y)$, there is an isotopy $H_{t}:\left(Z, Z_{0}\right) \rightarrow$ $\left(Z, Z_{0}\right)$ such that

(1) $H_{0}=1 ; H_{t} \mid(X \cup Y \cup P)=1(0 \leqq t \leqq 1)$.

(2) $H_{1}\left(V_{0}\right)=W_{0}$.

Proof. Let $U_{0}$ be a regular neighborhood of $X \bmod Y$ in $Z_{0}$ such that $\left(U_{0}-Y\right)$ $\subset\left[\operatorname{Int}_{z_{0}}\left(V_{0}\right) \cap \operatorname{Int}_{z_{0}}\left(W_{0}\right)\right]$. Then $\mathrm{Cl}\left(V_{0}-X\right)$ and $\mathrm{Cl}\left(W_{0}-X\right)$ are regular neighborhoods of $U_{0} \bmod (X \cup Y)$ in $Z_{0}$, using (7.3). Because $\left(P \cap Z_{0}\right)<(X \cup Y)$, the preceding lemma implies that there exist $V$ and $W$ such that $\left(V, V_{0}\right)$ and $\left(W, W_{0}\right)$ are regular neighborhoods of $U_{0} \bmod (X \cup Y \cup P)$ in $\left(Z, Z_{0}\right)$. Therefore there is an isotopy $H_{t}$ of $\left(Z, Z_{0}\right)$ onto $\left(Z, Z_{0}\right)$ such that $H_{0}=1, H_{t} \mid(X \cup Y \cup P)=1$ and $H_{1}\left(\mathrm{Cl}\left(V_{0}-X\right)\right)=\mathrm{Cl}\left(W_{0}-X\right)$. Then

$$
H_{1}\left(V_{0}\right)=H_{1}\left(\mathrm{Cl}\left(V_{0}-X\right)\right) \cup X_{R}=\mathrm{Cl}\left(W_{0}-X\right) \cup X_{R}=W_{0}, \quad \text { q.e.d. }
$$

There are important situations (some of which arise in \$9) where we wish to know the conclusion of (7.7) but do not know that every triangulation of $Z_{0}$ extends to one of $Z$. One such circumstance is covered by

Proposition 7.8. Suppose that $V_{0}$ and $W_{0}$ are regular neighborhoods of $X \bmod Y$ in $Z_{0}$ where $X, Y<Z_{0}<Z$. Suppose further that $P<Z,(Z-P)$ is a manifold, $\left(Z_{0}-P\right)$ is an open subset of $\partial(Z-P)$, and $\left(P \cap Z_{0}\right)<(X \cup Y)$. Then there is a homeomorphism $G:\left(Z, Z_{0}\right) \rightarrow\left(Z, Z_{0}\right)$ such that $G \mid(X \cup Y \cup P)=1$ and $G\left(V_{0}\right)=W_{0}$.

REMARK. As in (7.7) the homeomorphism we construct is isotopic to the identity.

Proof. Let $g: Z_{0} \rightarrow Z_{0}$ be a homeomorphism fixed on $(X \cup Y)$ such that $g\left(V_{0}\right)=W_{0}$. We may assume, by (3.4), that $g=g_{1} g_{2} \cdots g_{q}$ where each $g_{j}$ is an $(n-1)$-move relative to $(X \cup Y)$. For a given $g_{j}$, let $\left\{Q_{i}^{n-1}\right\}$ be the family of balls with disjoint interiors on which $g_{j}$ is supported, where

$$
\left(Q_{i}^{n-1} \cap P\right)<\left(Q_{i}^{n-1} \cap(X \cup Y)\right)<\partial Q_{i}^{n-1} .
$$

By the definition of a move we may choose a single triangulation of $Z_{0}$-and then one of $Z$-in which $P, X, Y$, and all the $Q_{i}^{n-1}$ are subcomplexes. Let $U$ be a 
relative second derived neighborhood of $\bigcup Q_{i}^{n-1} \bmod \left(P \cup \bigcup \partial Q_{i}^{n-1}\right)$ in $Z$. Then $U=\bigcup Q_{i}^{n}$ where $Q_{i}^{n}$ is a regular neighborhood of $Q_{i}^{n-1} \bmod \left(P \cup \partial Q_{i}^{n-1}\right)$ in $Z$. By construction $Q_{i}^{n}$ and $Q_{k}^{n}$ have disjoint interiors if $i \neq k$.

We claim that $Q_{i}^{n}$ is an $n$-ball. For, let $Z_{1}=Z \cup v Q_{i}^{n-1}$. Then Int $Q_{i}^{n-1}$ is locally bicollared in $Z_{1}$ since $\left(Z_{0}-P\right)$ is an open subset of $\partial(Z-P)$. Hence it follows from the stellar neighborhood theorem and (7.4) that $v Q_{i}^{n-1}$ and $\left(v Q_{i}^{n-1} \cup Q_{i}^{n}\right)$ are regular neighborhoods of $v\left(\partial Q_{i}^{n-1}\right) \bmod \left(\partial Q_{i}^{n-1}\right)$ in $Z_{1}$. But the closure of the difference of these regular neighborhoods is precisely $Q_{i}^{n}$. Thus (7.5) implies that $Q_{i}^{n}$ is homeomorphic to a regular neighborhood of $\left(\sigma^{n-1} \times 0\right) \bmod \left(\dot{\sigma}^{n-1} \times 0\right)$ in $\sigma^{n-1} \times I$. (Here we are using the fact that $\operatorname{Bdy}_{z_{1}}\left(Q_{i}^{n} \cup v Q_{i}^{n-1}\right) \simeq \operatorname{Bdy}_{z_{1}}\left(v Q_{i}^{n-1}\right)=$ $Q_{i}^{n-1} \simeq \sigma^{n-1}$.) But, choosing a triangulation of $\left(\sigma^{n-1} \times I\right)$ in which $\sigma=\left(\sigma^{n-1} \times 0\right)$ is a simplex whose link is the vertex $w$, we have $N\left(\sigma-\dot{\sigma},(\sigma \times I)^{\prime}\right)=b(w \sigma) \sigma^{\prime}$. Thus $Q_{i}^{n}$ is an $n$-ball.

Now $Q_{i}^{n-1}$ is a face of $Q_{i}^{n}$ and $g_{j} \mid Q_{i}^{n-1}$ is a homeumorphism of $Q_{i}^{n-1}$ onto itself which is the identity on $\partial Q_{i}^{n-1}$. Hence $g_{j} \mid Q_{i}^{n-1}$ extends to a homeomorphism $G_{j}: Q_{i}^{n} \rightarrow Q_{i}^{n}$ such that $g_{j} \mid\left(\partial Q_{i}^{n}-Q_{i}^{n-1}\right)=1$. This defines $G_{j}$ on $\cup Q_{i}^{n}$, and we extend $G_{j}$ to all of $Z$ by defining $G_{j} \mid(Z-U)=1$. The homeomorphism $G=G_{1} G_{2}$ $\cdots G_{q}$ has the desired properties, q.e.d.

PROPOSITION 7.9. Assume that $X, Y, Z_{0}<Z$ and that $V_{0}$ is a regular neighborhood of $X \cap Z_{0} \bmod Y \cap Z_{0}$ in $Z_{0}$. If either, (a) every triangulation of $Z_{0}$ extends to a triangulation of $Z$, or (b) $Z-(X \cup Y)$ is a manifold and $Z_{0}-(X \cup Y)$ is an open subset of its boundary, then there is a polyhedron $V<Z$ such that $\left(V, V_{0}\right)$ is a regular neighborhood of $X \bmod Y$ in $\left(Z, Z_{0}\right)$.

Proof. Let $\left(W, W_{0}\right)$ be any regular neighborhood of $X \bmod Y$ in $\left(Z, Z_{0}\right)$. By (7.7) or (7.8) there is a homeomorphism $h$ of $\left(Z, Z_{0}\right)$ onto itself such that $h \mid(X \cup Y)$ $=1$ and $h\left(W_{0}\right)=V_{0}$. Set $V=h(W)$, q.e.d.

Proposition 7.10. Suppose that $X, Y, Z_{0}<Z$. Then

(a) If $\left(V, V_{0}\right)$ is a regular neighborhood of $X \bmod Y$ in $\left(Z, Z_{0}\right)$ then $V$ is a regular neighborhood of $\left(X \cup V_{0}\right) \bmod \left(Y \cup \dot{V}_{0}\right)$ in $Z$. (Here $\dot{V}_{0} \equiv \operatorname{Bdy}_{Z_{0}} V_{0}$. $)$

(b) If $V_{0}$ is a regular neighborhood of $\left(X \cap Z_{0}\right) \bmod \left(Y \cap Z_{0}\right)$ in $Z_{0}$ which extends to some regular neighborhood $\left(W, V_{0}\right)$ of $X \bmod Y$ in $\left(Z, Z_{0}\right)$, if $\left(V \cap Z_{0}\right)-Y$ $=V_{0}-Y$, and if $V$ is a regular neighborhood of $\left(X \cup V_{0}\right) \bmod \left(Y \cup \dot{V}_{0}\right)$ in $Z$ then $\left(V, V_{0}\right)$ is a regular neighborhood of $X \bmod Y$ in $\left(Z, Z_{0}\right)$.

Remark. The hypothesis of (b) can sometimes be verified using (7.9).

Proof of (a). Let $X_{1}=\left(X \cup V_{0}\right)$ and $Y_{1}=\left(Y \cup \dot{V}_{0}\right)$. Then $V$ is a topological neighborhood of $\left(X_{1}-Y_{1}\right)$ and $\left(V \cap Y_{1}\right)=\left(Y_{1}\right)_{R}$. Choose a full triangulation $\left(J, J_{0}, K, L ; h\right)$ of $\left(Z, Z_{0}, X, Y\right)$ such that $\left(V, V_{0}\right)=h\left(N\left(K-L, J^{\prime}\right), N\left(K-L, J_{0}^{\prime}\right)\right)$. Set $K_{1}=K^{\prime} \cup N\left(K-L, J_{0}^{\prime}\right)$ and $L_{1}=L^{\prime} \cup \dot{N}\left(K-L, J_{0}^{\prime}\right)$. Then (except for a technicality which we explain below), $\left(J^{\prime}, K_{1}, L_{1} ; h\right)$ is a full triangulation of $\left(Z, X_{1}, Y_{1}\right)$ such that

$$
V=h N\left(K-L, J^{\prime}\right)=h N\left(K_{1}-L_{1}, J^{\prime}\right) \quad \text { and } \quad \dot{N}\left(K-L, J^{\prime}\right)=\dot{N}\left(K_{1}-L_{1}, J^{\prime}\right) .
$$


The last equation can be used to prove that $\dot{N}\left(K_{1}-L_{1}, J^{\prime}\right)-L_{1}$ is locally collared in $N\left(K_{1}-L_{1}, J^{\prime}\right)$ and in $C\left(K_{1}-L_{1}, J^{\prime}\right)$. Therefore we may apply the stellar neighborhood theorem (6.1c) and conclude that $V$ is a regular neighborhood of $X_{1} \bmod Y_{1}$ in $Z$.

The assertions made in the preceding paragraph can be verified in a straightforward manner, except for the claim that $L_{1}$ is full in $J^{\prime}$. This is not true in general but is true if

$$
\left(^{*}\right): L \cap N\left(K-L, J_{0}\right)<\left(J_{0} \cap L\right)_{R} \equiv\left[\mathrm{Cl}\left(J_{0} \cap K-J_{0} \cap L\right)\right] \cap L .
$$

Suppose that $\left(\bar{J}, \bar{J}_{0}, \bar{K}, \bar{L} ; \bar{g}\right)$ is any full triangulation of $\left(Z, Z_{0}, X, Y\right)$. Let $g$ be a homeomorphism of $\left(Z, Z_{0}\right)$ fixed on $X \cup Y$ and carrying

$$
\left(\bar{g} N\left(K-L, J^{\prime}\right), \bar{g} N\left(K-L, J_{0}^{\prime}\right)\right)
$$

onto $\left(V, V_{0}\right)$. Then, by choosing $\left(J, J_{0}, K, L ; h\right)=\left(\bar{J}^{\prime}, \bar{J}_{0}^{\prime}, \bar{K}^{\prime}, \bar{L}^{\prime} ; g \bar{g}\right)$ at the outset, we can insure that $\left(^{*}\right)$ is satisfied.

Proof of (b). Part (a) implies that $W$ is a regular neighborhood of $\left(X \cup V_{0}\right) \bmod \left(Y \cup \dot{V}_{0}\right)$ in $Z$. By hypothesis $V$ is also such a regular neighborhood. In fact, by (7.1), (2.13), and the assumption that $\left[\left(V \cap Z_{0}\right)-Y\right]=\left[V_{0}-Y\right]$, these are regular neighborhoods of $\left(X \cup V_{0}\right) \bmod \left(Y \cup\left(\mathrm{Cl}\left(Z_{0}-V_{0}\right)\right)\right)$ in $Z$. Thus there is a homeomorphism $h: Z \rightarrow Z$ such that $h \mid\left(Z_{0} \cup X \cup Y\right)=1$ and $h(W)=V$. Hence $\left(V, V_{0}\right)$ is a regular neighborhood of $X \bmod Y$ in $\left(Z, Z_{0}\right)$, q.e.d.

\section{Collapsibility and collapsible retractions.}

Definition. Suppose that $Y<X<Z$. Then we write $Z \searrow X(\bmod Y)$ if there exists a finite sequence $Z=Z_{0}>Z_{1}>Z_{2}>\cdots>Z_{q}=X$ such that

(i) $Z_{i}=Z_{i+1} \cup B_{i}$ where $B_{i}$ is an $\left(n_{i}\right)$-ball, $n_{i}>0$.

(ii) $F_{i}=\left(B_{i} \cap Z_{i+1}\right)$ is an $\left(n_{i}-1\right)$-face of $B_{i}$.

(iii) $\left(F_{i} \cap Y\right)<\partial F_{i}$.

Definition. If $Y<X<Z$ then $p: Z \rightarrow X$ is a collapsible retraction, $\bmod Y$, of $Z$ onto $X$ if

(i) $p$ is a retraction of $Z$ onto $X$.

(ii) $p^{-1}(x)$ is a compact collapsible polyhedron, for each $x$ in $X$.

(iii) $p^{-1}(Y)=Y$.

The purpose of this section is to prove

THEOREM 8.1. If $Y<X<Z$ is a triple of compact polyhedra then $Z \searrow X(\bmod Y)$ if and only if there is a collapsible retraction $\bmod Y$ of $Z$ onto $X$.

In the light of (5.1) this theorem implies

COROLlaRY 8.2. If $V$ is compact and $V$ is a regular neighborhood of $X \bmod Y$ in $Z$ then $V \searrow X_{R}\left(\bmod Y_{R}\right)$.

REMARK. At the end of this section we discuss the appropriate generalization of (8.1) to noncompact triples. The importance of collapsing relative to $Y_{R}$ is indicated by Theorem 9.1 and the example given in [T]. 
LemMA 8.3. If $Z \searrow X$ and $D^{n}$ is an $n$-ball then

$$
\left(Z \times D^{n}\right) \searrow\left(Z \times \partial D^{n}\right) \cup\left(X \times D^{n}\right)
$$

Proof. Let $Z=Z_{0} \searrow \cdots \searrow Z_{q}=X$ be a sequence of elementary collapses. Suppose that

$$
\left(Z \times D^{n}\right) \searrow\left(Z \times \partial D^{n}\right) \cup\left(Z_{i} \times D^{n}\right)=\left[\left(Z \times \partial D^{n}\right) \cup\left(Z_{i+1} \times D^{n}\right)\right] \cup\left(B_{i} \times D^{n}\right)
$$

where $Z_{i}=Z_{i+1} \cup B_{i},\left(B_{i} \cap Z_{i+1}\right)=F_{i}$ and $G_{i}=\mathrm{Cl}\left(\partial B_{i}-F_{i}\right)$. Notice that

$$
\begin{aligned}
{\left[\left(Z \times \partial D^{n}\right) \cup\left(Z_{i+1} \times D^{n}\right)\right] \cap\left(B_{i} \times D^{n}\right) } & =\left(B_{i} \times \partial D^{n}\right) \cup\left(F_{i} \times D^{n}\right) \\
= & \mathrm{Cl}\left[\partial\left(B_{i} \times D^{n}\right)-\left(G_{i} \times D^{n}\right)\right], \text { a face of } B_{i} \times D^{n} .
\end{aligned}
$$

Thus $\left(Z \times D^{n}\right) \searrow\left(Z \times \partial D^{n}\right) \cup\left(Z_{i+1} \times D^{n}\right)$. The proof now proceeds by induction on the number of collapses, q.e.d.

LEMMA 8.4. If $J$ and $K$ are finite simplicial complexes such that $J \searrow K$ and if $\sigma$ is $a$ simplex, then $\sigma J \searrow \dot{\sigma} J \cup \sigma K(\bmod \sigma)$.

The proof is similar to the proof of (8.3).

LEMMA 8.5. If $V$ is compact and is a regular neighborhood of $X \bmod Y$ in $Z$ then $V \searrow X_{R}$.

Proof. Let $(J, K, L ; h)$ be a full triangulation of $(Z, X, Y)$ with $V=h N\left(K-L, J^{\prime}\right)$. Then $N\left(K-L, J^{\prime}\right)=N\left(K_{R}, N\left(K-L, J^{\prime}\right)\right)$. If $A$ is a simplex of $\dot{N}\left(K_{R}, N\left(K-L, J^{\prime}\right)\right)$, then $A=b\left(\sigma_{0}\right) \cdots b\left(\sigma_{r}\right)$ where $\sigma_{i}=\alpha_{i} \beta_{i} \gamma_{i}$, as usual, and $\beta_{0} \neq \varnothing \neq \gamma_{0}$. Hence (see 2.10)

$$
\operatorname{Lk}\left(A, N\left(K-L, J^{\prime}\right)\right) \cap K_{R}^{\prime}=\left(\alpha_{0} \beta_{0}\right)^{\prime} \searrow 0 .
$$

By Theorem 2 of [W], $N\left(K-L, J^{\prime}\right) \searrow K_{R}^{\prime}$, q.e.d.

Proof of the sufficiency in Theorem 8.1. We assume that $Y<X<Z$ and that $p: Z \rightarrow X$ is a collapsible retraction $\bmod Y$. We proceed by induction on $q=$ dimension $Y$.

Suppose that $Y$ is empty $(q=-1)$. Choose a triangulation $(J, K)$ of $(Z, X)$ (we suppress the triangulating homeomorphism) such that $p: J \rightarrow K$ is simplicial. Choose first and second deriveds such that $p: J^{\prime} \rightarrow K^{\prime}$ and $p: J^{\prime \prime} \rightarrow K^{\prime \prime}$ are also simplicial. Let $\sigma$ be a principal simplex of $K$. We shall show that $p^{-1}(\sigma)$ $\searrow \sigma \cup p^{-1}(\dot{\sigma})$. Repeated application of this result then yields a collapse of $J$ onto $K$.

Let $\sigma_{0}=N\left(b(\sigma), \sigma^{\prime \prime}\right)$. Let $C$ denote the collar $N\left(\dot{\sigma}^{\prime \prime}, \sigma^{\prime \prime}\right)$ and let $C \cap \sigma_{0}=\dot{\sigma}_{0}$. Notice first that

$$
\left(p^{-1}\left(\sigma_{0}\right), p^{-1}\left(\dot{\sigma}_{0}\right), \sigma_{0}\right) \simeq\left(\sigma_{0} \times p^{-1} b(\sigma), \dot{\sigma}_{0} \times p^{-1} b(\sigma), \sigma_{0} \times b(\sigma)\right),
$$

using the well-known fact that a simplicial mapping is a product on the inverse image of the interior of a top dimensional simplex of the range. Therefore we may apply (8.3) and conclude that $p^{-1}\left(\sigma_{0}\right) \searrow\left(p^{-1}\left(\dot{\sigma}_{0}\right) \cup \sigma_{0}\right)$. Now notice that 
$\left(p^{-1} C, C\right)=\left(N\left(p^{-1}(\dot{\sigma})^{\prime \prime}, p^{-1}(\sigma)^{\prime \prime}\right), C\right)$ is a regular neighborhood of $p^{-1}(\dot{\sigma})$ in $\left(p^{-1}(\sigma), \sigma\right)$. Hence, by $(7.10), p^{-1}(C)$ is a regular neighborhood of

$$
\left(p^{-1}(\dot{\sigma}) \cup C\right) \bmod \dot{\sigma}_{0} \quad \text { in } p^{-1}(\sigma) .
$$

Therefore, by (8.5), $p^{-1}(C) \searrow p^{-1}(\dot{\sigma}) \cup C$. Putting these steps together we have

$$
\begin{aligned}
p^{-1}(\sigma) & =p^{-1}\left(\sigma_{0}\right) \cup p^{-1}(C), \quad\left[p^{-1}\left(\sigma_{0}\right) \cap p^{-1}(C)=p^{-1}\left(\dot{\sigma}_{0}\right)\right] \\
& \searrow \sigma_{0} \cup p^{-1}(C), \quad\left[\sigma_{0} \cap p^{-1}(C)=\dot{\sigma}_{0}\right] \\
& \searrow \sigma_{0} \cup\left(C \cup p^{-1} \dot{\sigma}\right) \\
& =\sigma \cup p^{-1} \dot{\sigma} .
\end{aligned}
$$

Suppose now that $q \geqq 0$ and the theorem is known for integers less than $q$. Let $(J, K, L)$ be a triangulation of $(Z, X, Y)$ such that $p: J \rightarrow K$ and $p: J^{\prime} \rightarrow K^{\prime}$ are simplicial. The hypotheses that $p^{-1} L=L$ implies $(2.15)$ that $p^{-1} \dot{N}\left(L^{\prime}, K^{\prime}\right)=\dot{N}\left(L^{\prime}, J^{\prime}\right)$ and that $p^{-1} \operatorname{Lk}\left(A, K^{\prime}\right)=\operatorname{Lk}\left(A, J^{\prime}\right)$, for each simplex $A<L^{\prime}$. Denote $I(A, S)$ $=\mathrm{Lk}\left(A, S^{\prime}\right) \cap \dot{N}\left(L^{\prime}, S^{\prime}\right)$. Then $p \mid I(A, J)$ is a collapsible retraction onto $I(A, K)$. So, using the result for $q=-1, I(A, J) \searrow I(A, K)$. Therefore (8.4) implies that $A I(A, J) \backslash A I(A, K) \cup \dot{A} I(A, J),(\bmod A)$. Moreover, if $A$ and $B$ are $q$-simplexes of $L^{\prime}$ then $[A I(A, J) \cap B I(B, J)]<[\dot{A} I(A, J) \cap \dot{B} I(B, J)]$ so that the collapses of $A I(A, J)$ and $B I(B, J)$ do not affect one another. Performing this collapse for each $q$-simplex $A<L^{\prime}$ we have

$$
\begin{aligned}
J^{\prime} & =C\left(L^{\prime}, J^{\prime}\right) \cup N\left(L^{\prime}, J^{\prime}\right)=C\left(L^{\prime}, J^{\prime}\right) \cup \cup\left\{A I(A, J) \mid A<L^{\prime}\right\} \\
& \searrow C\left(L^{\prime}, J^{\prime}\right) \cup K^{\prime} \cup \cup\left\{A I(A, J) \mid A<L^{\prime}, \operatorname{dim} A<q\right\}\left(\bmod L^{\prime}\right) \\
& =K^{\prime} \cup p^{-1}\left[C\left(L^{\prime}, K^{\prime}\right) \cup \bigcup\left\{A I(A, K) \mid A<L^{\prime}, \operatorname{dim} A<q\right\}\right] \\
& \searrow K^{\prime}\left(\bmod L^{\prime}\right), \text { by induction hypothesis. }
\end{aligned}
$$

This completes the proof of the sufficiency in Theorem 8.1.

LEMMA 8.6. The composition of collapsible retractions is a collapsible retraction.

Proof. Suppose that $p_{1}: X \rightarrow X_{1}$ and $p_{2}: X_{1} \rightarrow X_{2}$ are collapsible retractions and that $x \in X_{2}$. Then

$$
\left(p_{2} p_{1}\right)^{-1}(x)=p_{1}^{-1}\left(p_{2}^{-1} x\right) \searrow p_{2}^{-1}(x) \searrow x .
$$

The first collapse comes from the assumption on $p_{1}$ and the sufficiency in (8.1). The second collapse comes from the assumption on $p_{2}$, q.e.d.

Proof of the necessity in (8.1). Assume that $Z \backslash X(\bmod Y)$. Thus $Z=Z_{0}$ $>\cdots>Z_{q}=X$ where $Z_{i}=Z_{i+1} \cup B_{i}$ and $B_{i} \cap Z_{i+1}=F_{i}$. Define the retraction $p_{i}: Z_{i} \rightarrow Z_{i+1}$ as follows. Triangulate $\partial F_{i}$. Triangulate $F_{i}$ as $v_{0}\left(\partial F_{i}\right)$ and triangulate $B_{i}$ as $v_{0} v_{1}\left(\partial F_{i}\right)$. Then $p_{i}$ is defined to be the identity on $Z_{i+1}$ and $p_{i}$ is defined on $B_{i}$ as the simplicial mapping such that $p_{i}\left(v_{0} v_{1}\right)=v_{0}$. Notice that $p_{i}^{-1}\left(\partial F_{i}\right)=\partial F_{i}$ and $p_{i}^{-1}(x)$ is a point or an arc for each $x \in Z_{i+1}$. Then $p=p_{q-1} \cdots p_{0}$ is a retraction of $Z$ onto $X$. Point inverses are collapsible by (8.6). The fact that $\left(Y \cap F_{i}\right)<\partial F_{i}$ implies that no $p_{i}$ ever brings a point outside of $Y$ into $Y$. Hence $p^{-1}(Y)=Y$, q.e.d. 
REMARK. Theorem 8.1 asserts the equivalence, in the compact case, of the existence of a collapse $X \searrow Y(\bmod Z)$ and the existence of a collapsible retraction, $\bmod Y$, of $Z$ onto $X$. It is the latter notion which we choose to emphasize in the general case. Notice however that the proof of (8.1) gives us the following information: If a given infinite collapsing process $\left({ }^{5}\right)$ induces a well-defined retraction $p$ such that, for each $x, p \mid p^{-1}(x)$ is the composition of finitely many collapsible retractions then $p$ is a collapsible retraction. On the other hand, if there is a collapsible retraction mod $Y^{q}$ of $Z$ onto $X^{n}$ then there is a composition of $n+q+2$ "collapses" taking $Z$ onto $X$, where a "collapse" consists of countably many disjoint, simultaneous finite collapses relative to $Y$.

9. Regular neighborhoods via collapsibility. The purpose of this section is to prove

THEOREM 9.1. If $X, Y, V<M$ and

(1) $M-(X \cup Y)$ is an $n$-manifold,

(2) $V$ is a topological neighborhood of $X-Y$ in $M$,

(3) $V \cap Y=Y_{R}$,

(4) $V-X$ is an n-manifold,

(5) there is a collapsible retraction, $\bmod Y_{R}$, of $V$ onto $X_{R}$, then

(a) there are regular neighborhoods $U$ and $U_{0}$ of $X \bmod Y$ in $M$, contained in $V$, and there is an isotopy $H$ of $V$ into itself such that $H \mid(V \times 0) \cup\left(U_{0} \times I\right)=1$ and $H_{1}(V)=U$,

(b) if $M-(X \cup Y)$ is a manifold without boundary then $V$ is a regular neighborhood of $X \bmod Y$ in $M$,

(c) if $M$ is actually a manifold and if $\mathrm{Cl}[(V \cap \partial M)-Y]$ is a regular neighborhood of $(X \cap \partial M) \bmod (Y \cap \partial M)$ in $\partial M$ then $V$ is a regular neighborhood of $X \bmod Y$ in $M$.

REMARK. One can combine (9.1), (7.10) and (7.9) to get the following program for recognizing regular neighborhoods in polyhedra via collapsibility. If $Z$ is an $n$ dimensional polyhedron let $Z_{1}$ denote the set of points of $Z$ whose links are not $(n-1)$-spheres (i.e. the intrinsic $(n-1)$-skeleton of $Z$ ). If $X, Y, V<Z$ let $V_{1}$ $=\mathrm{Cl}\left[\left(V \cap Z_{1}\right)-Y\right]$. Then, by (7.10b), $V$ is a regular neighborhood of $X \bmod Y$ in $Z$ if (a) $V_{1}$ is a regular neighborhood of $\left(X \cap Z_{1}\right) \bmod \left(Y \cap Z_{1}\right)$ in $Z_{1}$, (b) $V_{1}$ extends to some regular neighborhood of $X \bmod Y$ in $\left(Z, Z_{1}\right)$, and (c) $V$ is a regular neighborhood of $\left(X \cup V_{1}\right) \bmod Y \cup \mathrm{Cl}\left(Z_{1}-V_{1}\right)$ in $Z$. Since $\left(Z-Z_{1}\right)$ is an $n$-manifold without boundary, condition (c) can be checked using (9.1b). Condition (b) is not always satisfied. One has to verify it directly or, if possible, apply (7.9). Condition (a) is just the recognition problem in lower dimension. Proceed inductively $\left({ }^{6}\right)$.

$\left(^{5}\right)$ E.g. the process of trace-finite collapse considered in [S].

$\left({ }^{6}\right)$ Since this was written J. Stallings [Notes on polyhedral topology, Tata Institute, 1967] and E. Akin (loc. cit.) have carried through similar but more efficient programs for compact polyhedra. 
EXAMPLE. The importance of condition (b) in the above remark is illustrated in the simple situation where $Z=\left(R^{2} \times 0\right) \cup([0,2] \times 0 \times[0,10])<R^{3}, X=\{(1,0,0)\}$, $Y=\varnothing$, and $V=([0,2] \times 0 \times[0,5]) \cup([-1,3] \times[-1,1] \times 0)$. An example where (c) fails was given in (5.5).

CONVENTION. If $Z$ is an arbitrary $q$-dimensional polyhedron then $\partial Z$ denotes the subpolyhedron consisting of points of $Z$ whose links are not $(q-1)$-spheres (i.e. the intrinsic $(q-1)$-skeleton of $Z$. Notice that $\partial Z$ underlies a subcomplex of any triangulation of $Z$.

Preliminaries to proving (9.1a). Let $(J, K, L ; h)$ be a triangulation of $\left(V, X_{R}, Y_{R}\right)$ such that $h^{-1} p h: J \rightarrow K$ is simplicial. To simplify the notation we write $p=h^{-1} p h$, when no confusion can occur. Choose a first derived $J^{\prime}$ such that $p: J^{\prime} \rightarrow K^{\prime}$ is also simplicial. The proofs given to derive the basic facts about dual cells when $J$ is a manifold (see $\$ 5$ of [C]) apply almost verbatim here, where $J-K$ is a manifold and the symbol " $\partial$ " has the broadened meaning given above. Thus, if $\sigma$ is an $i$-simplex of $K-L$, we know

(A) $D(\sigma, p)-D(\sigma, K)$ is an (n-i)-manifold (possibly empty).

(B) $\partial D(\sigma, p)=\dot{D}(\sigma, p) \cup D(\sigma, p \mid \partial J)$.

(C) $\dot{D}(\sigma, p)-D(\sigma, K)$ and $D(\sigma, p \mid \partial J)-D(\sigma, K)$ are $(n-i-1)$-manifolds.

(One or both may be empty.)

Analogous statements hold for $D(\sigma, J), \dot{D}(\sigma, J)$ and $D(\sigma, \partial J)$.

The idea of the proof of (a) is to retriangulate $|J|$ as a stellar neighborhood of $K^{\prime} \bmod L^{\prime}$. First we use the fact that $p$ is a collapsible retraction to triangulate each dual cell $D(\sigma, p), \sigma<K-L$, as a cone which stretches from the subcone $D(\sigma, K)$ out to $\partial J$. This yields a new triangulation of $\left|C\left(L^{\prime}, J^{\prime}\right)\right|$. Then we use the hypothesis that $p^{-1} L=L$ to extend this triangulation over $\left|N\left(L^{\prime}, J^{\prime}\right)\right|$. The crux of the first part is given by

LEMmA 9.2. If, in the above situation, $\sigma<K-L$ then there is a homeomorphism

$$
f:(v C(b(\sigma), \partial D(\sigma, p)), v \dot{D}(\sigma, K)) \rightarrow(D(\sigma, p), D(\sigma, K))
$$

such that

(i) $f \mid C(b(\sigma), \partial D(\sigma, p))=1$,

(ii) $f(v)=b(\sigma)$ and $f \mid v \dot{D}(\sigma, K)=(f \mid v) * 1_{\dot{D}(\sigma, K)}$ (see Figure 3).

REMARK. $b(\sigma)<\partial D(\sigma, p) \Leftrightarrow \sigma<\partial J$. If $\sigma \nless \partial J$ then

$$
C(b(\sigma), \partial D(\sigma, p))=\partial D(\sigma, p) .
$$

Similar assertions hold for $\partial D(\sigma, J)$.

Proof of the lemma. Since $p \mid D(\sigma, p)$ is a collapsible retraction of $D(\sigma, p)$ onto $D(\sigma, K)$ it follows $(8.1)$ that $D(\sigma, p) \searrow D(\sigma, K)$. We subdivide to get a simplicial collapse and then apply Whitehead's shelling procedure [W, Theorem 23n] to conclude that $D(\sigma, p)$ is homeomurphic, keeping $D(\sigma, K)$ fixed, to a regular neighborhood of $D(\sigma, K)$ in $D(\sigma, p)$. (Whitehead's procedure works because 


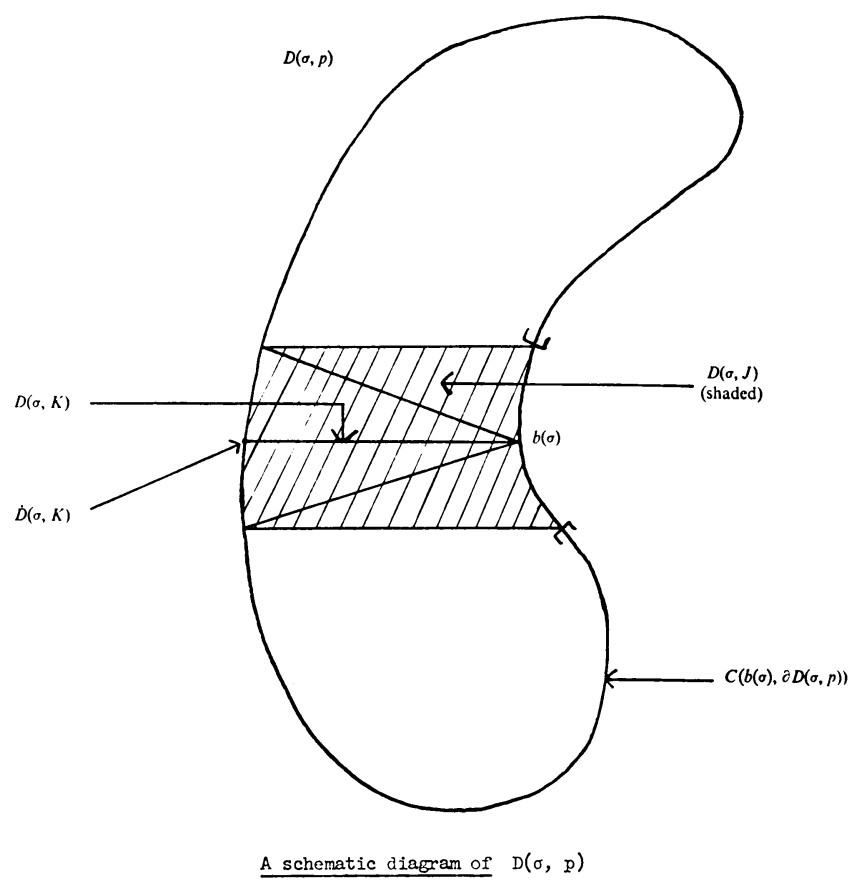

FIGURE 3

$[D(\sigma, p)-D(\sigma, K)]$ is a manifold. This procedure only involves the subtracting away of balls which are stars of barycenters of simplexes deleted during the simplicial collapse. Hence it does not matter whether $D(\sigma, p)$ is a manifold along $D(\sigma, K)$.)

We claim that $D(\sigma, J)$ is also homeomorphic, keeping $D(\sigma, K)$ fixed, to a regular neighborhood of $D(\sigma, K)$ in $D(\sigma, p)$. For one checks easily that

$$
D(\sigma, J)=N(D(\sigma, K), D(\sigma, p))=N(D(\sigma, K), D(\sigma, J))
$$

and

$$
\begin{aligned}
\dot{N}(D(\sigma, K), D(\sigma, p)) & =\dot{N}(D(\sigma, K), D(\sigma, J)) \\
& <[\dot{D}(\sigma, J)-D(\sigma, K)] \\
& <[\partial D(\sigma, J)-D(\sigma, K)] .
\end{aligned}
$$

But $\dot{N}(D(\sigma, K), D(\sigma, J))$ is a manifold by (5.4), being simplicially isomorphic to $C\left(\mathrm{Lk}(\sigma, K)^{\prime}, \mathrm{Lk}(\sigma, J)^{\prime}\right)$. Thus it is a codimension zero submanifold of $[\partial D(\sigma, J)$ $-D(\sigma, K)]$ and so is locally collared in $[D(\sigma, J)-D(\sigma, K)]$. The claim follows from the stellar neighborhood theorem (6.1a).

Invoking the uniqueness of regular neighborhoods, there is a homeomorphism $f_{0}: D(\sigma, p) \rightarrow D(\sigma, J)$ such that $f_{0} \mid D(\sigma, K)=1$. We shall modify $f_{0}$ to get a homeomorphism which also takes $C(b(\sigma), \partial D(\sigma, p))$ onto $C(b(\sigma), \partial D(\sigma, J))$. 
Notice that

$$
\begin{aligned}
D(\sigma, K) \cap \partial D(\sigma, p) & =D(\sigma, K) \cap[\dot{D}(\sigma, p) \cup D(\sigma, p \mid \partial J)] \\
& =D(\sigma, K) \cap\left[p^{-1} \dot{D}(\sigma, K) \cup\left(p^{-1} D(\sigma, K) \cap \partial J\right)\right] \\
& =\dot{D}(\sigma, K) \cup(D(\sigma, K) \cap \partial J) \\
& =\dot{D}(\sigma, K) \cup D(\sigma, K \cap \partial J) .
\end{aligned}
$$

Similarly

$$
D(\sigma, K) \cap \partial D(\sigma, J)=\dot{D}(\sigma, K) \cup D(\sigma, K \cap \partial J) .
$$

We claim that $N(b(\sigma), \partial D(\sigma, p))=N_{\sigma}$ is a regular neighborhood of

$$
D(\sigma, K \cap \partial J) \bmod \dot{D}(\sigma, K)
$$

in $\partial D(\sigma, p)$. For the complex $D(\sigma, K \cap \partial J)$ is a subcone of $N_{\sigma}$ and it is easy to see that

$$
\begin{gathered}
D(\sigma, K \cap \partial J) \cap \dot{D}(\sigma, K)=D(\sigma, K \cap \partial J) \cap \dot{N}_{\sigma}, \\
N_{\sigma}=N(D(\sigma, K \cap \partial J)-\dot{D}(\sigma, K), \partial D(\sigma, p)) .
\end{gathered}
$$

Further, $\left[\dot{N}_{\sigma}-\dot{D}(\sigma, K)\right]$ is locally collared in the cone $N_{\sigma}$ and so, as a codimension one submanifold of the open manifold [ $\partial D(\sigma, p)-D(\sigma, K)]$, is also locally collared in $C(b(\sigma), \partial D(\sigma, p))$. Thus the claim follows from the stellar neighborhood theorem (6.1c). Similarly $N(b(\sigma), \partial D(\sigma, J))$ is a regular neighborhood of

$$
D(\sigma, K \cap \partial J) \bmod \dot{D}(\sigma, K) \text { in } \partial D(\sigma, J) .
$$

But $f_{0} \mid D(\sigma, K)=1$. Thus the preceding paragraph tells us that $f_{0} N(b(\sigma), \partial D(\sigma, p))$ and $N(b(\sigma), \partial D(\sigma, J))$ are each regular neighborhoods of $D(\sigma, K \cap \partial J) \bmod \dot{D}(\sigma, K)$ in $\partial D(\sigma, J)$. Since $D(\sigma, K \cap \partial J) \cup \dot{D}(\sigma, K)=D(\sigma, K) \cap \partial D(\sigma, J)$ it follows from (7.8) that there is a homeomorphism $f_{1}$ of $D(\sigma, J)$ onto itself such that

and

$$
f_{1} \mid D(\sigma, K)=1
$$

$$
f_{1} f_{0} N(b(\sigma), \partial D(\sigma, p))=N(b(\sigma), \partial D(\sigma, J)) .
$$

The homeomorphism $f_{2}=f_{1} f_{0}$ of $D(\sigma, p)$ onto $D(\sigma, J)$ takes $C(b(\sigma), \partial D(\sigma, p))$ onto $C(b(\sigma), \partial D(\sigma, J))$ and is the identity on $D(\sigma, K)$.

Since $D(\sigma, J)=b(\sigma) \dot{D}(\sigma, J)=b(\sigma) C(b(\sigma), \partial D(\sigma, J))$ is a cone with $D(\sigma, K)$ as a subcone, $D(\sigma, p)$ is, piecewise linearly, also such a cone. Explicitly, define

$$
f:(v C(b(\sigma), \partial D(\sigma, p)), v \dot{D}(\sigma, K)) \rightarrow(D(\sigma, p), D(\sigma, K))
$$

by setting $f(v)=b(\sigma)$ and defining

$$
f=f_{2}^{-1} \circ\left[(f \mid v) *\left(f_{2} \mid C(b(\sigma), \partial D(\sigma, p))\right)\right] .
$$

This completes the proof of the lemma.

Proof of (9.1a). Let $\bar{L}$ and $Q$ be joinable complexes and let $\bar{C}$ be joinable with $\bar{L} * Q$, where $\bar{L} \cong L^{\prime}, Q \cong C\left(L^{\prime}, K^{\prime}\right)$ and $\bar{C} \cong C\left(K^{\prime}, \partial J^{\prime}\right)$. Every simplex of $K^{\prime}$ is of the 
form $A B$ where $A<L^{\prime}$ and $B<C\left(L^{\prime}, K^{\prime}\right)$, so $K^{\prime}$ is naturally isomorphic to a subcomplex $\bar{K}<\bar{L} * Q$. Let $f: \bar{K} \cup \bar{C} \rightarrow K^{\prime} \cup C\left(K^{\prime}, \partial J^{\prime}\right)$ be a simplicial isomorphism such that $f(\bar{K})=K^{\prime}$ and $f(\bar{L})=L^{\prime}$. In general, if $S<K^{\prime}$ we set $\bar{S}=f^{-1}(S)$. We shall construct a subcomplex $\bar{J}<\bar{K} * \bar{C}$ and a homeomorphism $g: \bar{J} \rightarrow J^{\prime}$ such that $(\bar{J}, \bar{K}, \bar{L} ; h g)$ is a stellar triangulation of $\left(V, X_{R}, Y_{R}\right)$ and $h g(\dot{N}(\bar{K}-\bar{L}, \bar{J})-\bar{L})$ is locally collared in $V$. Then (9.1a) will follow from the stellar neighborhood theorem.

Let $J_{i}<J^{\prime}(-1 \leqq i \leqq q=\operatorname{dim}(K-L))$ be given by

$$
J_{i}=K^{\prime} \cup C\left(K^{\prime}, \partial J^{\prime}\right) \cup \bigcup\left\{D\left(\sigma^{j}, p\right) \mid \sigma^{j}<K-L, j \geqq q-i\right\} .
$$

Notice that $J_{q}=K^{\prime} \cup C\left(L^{\prime}, J^{\prime}\right)$ because

$$
\begin{aligned}
C\left(L^{\prime}, J^{\prime}\right) & =p^{-1} C\left(L^{\prime}, K^{\prime}\right), \quad \text { by }(2.14) \\
& =p^{-1} \cup\{D(\sigma, K) \mid \sigma<K-L\} \\
& =\bigcup\{D(\sigma, p) \mid \sigma<K-L\} .
\end{aligned}
$$

Then $\left(\bar{J}_{-1}, g_{-1}\right) \equiv(\bar{K} \cup \bar{C}, f)$ is a triangulation of $J_{-1}$. Proceeding inductively assume that a complex $\bar{J}_{j}<\bar{K} * \bar{C}$ and a homeomorphism $g_{j}$ have been chosen for each $j<i(i \leqq q)$ so that

(i) $\left(\bar{J}_{j}, g_{j}\right)$ is a triangulation of $J_{j}$.

(ii) If $k<j$ then $\bar{J}_{k}<\bar{J}_{j}$ and $g_{k}=g_{j} \mid \bar{J}_{k}$.

(iii) If $\sigma=\sigma^{q-j}<K-L$ then $g_{j}^{-1} D(\sigma, p)$ is a subcomplex of $\bar{J}_{j}$-denoted $\bar{D}(\sigma, p)$ such that every principal simplex of $\bar{D}(\sigma, p)$ is the join of a nonempty simplex in $\bar{D}(\sigma, K)$ and a simplex in $\bar{C}$.

In order to extend $\left(\bar{J}_{i-1}, g_{i-1}\right)$ to a triangulation which covers each $D\left(\sigma^{q-i}, p\right)$, notice (writing $\sigma=\sigma^{q-i}$ ) that

$$
\begin{aligned}
D(\sigma, p) \cap J_{i-1}= & D(\sigma, K) \cup\left[D(\sigma, p) \cap C\left(K^{\prime}, \partial J^{\prime}\right)\right] \cup \dot{D}(\sigma, p) \\
= & D(\sigma, K) \cup\left[D(\sigma, p) \cap C\left(K^{\prime}, \partial J^{\prime}\right)\right] \cup[\dot{D}(\sigma, p) \cup \dot{D}(\sigma, p \mid \partial J)] \\
= & D(\sigma, K) \cup \dot{D}(\sigma, p) \cup \dot{D}(\sigma, p \mid \partial J) \\
& \cup\{b(\tau) \cdots b(\psi) \mid \sigma<p(\tau), \tau \nless K, \psi<\partial J\} \\
= & D(\sigma, K) \cup \dot{D}(\sigma, p) \cup C(b(\sigma), D(\sigma, p \mid \partial J)) \\
= & D(\sigma, K) \cup C(b(\sigma), \dot{D}(\sigma, p) \cup D(\sigma, p \mid \partial J)) \\
= & D(\sigma, K) \cup C(b(\sigma), \partial D(\sigma, p)) .
\end{aligned}
$$

Because $C(b(\sigma), \partial D(\sigma, p))$ is the union of the last two summands of the first equation, we see from (i)-(iii) that its inverse image underlies a subcomplexcall it $T$-of $J_{i-1}$. Also from the first equation, $T \cap \bar{K}=g_{i-1}^{-1} \dot{D}(\sigma, K)$, so that $\bar{b}(\sigma) T<\bar{D}(\sigma, K) *(T \cap \bar{C})$ is a subcomplex of $\bar{K} * \bar{C}$. We claim that $(\bar{b}(\sigma) T) \cap \bar{J}_{i-1}$ $=\bar{D}(\sigma, K) \cup T$. This is because any simplex of $\bar{J}_{i-1}$ which meets both $\bar{C}$ and $\bar{K}$ lies in some $\bar{D}(\tau, p)$, where $\operatorname{dim} \tau>i$, and thus, by (iii), cannot contain $\bar{b}(\sigma)$. We define $\bar{D}(\sigma, p)=\bar{b}(\sigma) T$. We extend $g_{i-1}$ to $g_{i}: \bar{D}(\sigma, p) \rightarrow D(\sigma, p)$ by stipulating that

$$
g_{i} \mid \bar{b}(\sigma) T=f \circ\left(k *\left(g_{i-1} \mid T\right)\right),
$$


where $k(\bar{b}(\sigma))=v$ and $f: v C(b(\sigma), \partial D(\sigma, p)) \rightarrow D(\sigma, p)$ is the homeomorphism given by the preceding lemma. Doing this for all $\sigma=\sigma^{q-i}<K-L$ we get a triangulation $\left(\bar{J}_{i}, g_{i}\right)$ of $J_{i}$ satisfying (i)-(iii).

The pair $\left(\bar{J}_{q}, g_{q}\right)$ constructed by this process is a new triangulation of $\left|K^{\prime} \cup C\left(L^{\prime}, J^{\prime}\right)\right|$. We proceed to extend this over $\left|N\left(L^{\prime}, J^{\prime}\right)\right|$. Let us denote

Then

$$
S=\left\{(A, \sigma) \mid \varnothing \neq A=b\left(\alpha_{0}\right) \cdots b\left(\alpha_{q}\right)<L^{\prime}, \alpha_{q}<\sigma, \sigma<K-L\right\} .
$$

Define $\bar{J}<\bar{K} * \bar{C}$ by

$$
\begin{aligned}
N\left(L^{\prime}, K^{\prime}\right) & =\bigcup\{A D(\sigma, K) \mid(A, \sigma) \in S\} \\
N\left(L^{\prime}, J^{\prime}\right) & =p^{-1} N\left(L^{\prime}, K^{\prime}\right), \quad \text { by }(2.14) \\
& =\bigcup\{A D(\sigma, p) \mid(A, \sigma) \in S\} .
\end{aligned}
$$

$$
\bar{J}=\bigcup\{\bar{A} \bar{D}(\sigma, p) \mid(A, \sigma) \in S\} \cup \bar{J}_{q} .
$$

Since $\bar{A} \bar{D}(\sigma, p)<\bar{A} * \bar{D}(\sigma, K) *(\bar{D}(\sigma, p) \cap \bar{C}), \bar{J}$ is a subcomplex of $\bar{K} * \bar{C}$. If $(A, \sigma) \in S$ and $\tau<K-L$ then $(\bar{A} \bar{D}(\sigma, p)) \cap \bar{D}(\tau, p)=[\bar{D}(\sigma, p) \cap \bar{D}(\tau, p)]=\bar{D}(\sigma \cdot \tau, p)$ where $\sigma \cdot \tau$ is the smallest simplex of $K$ containing both $\sigma$ and $\tau$ and $\bar{D}(\sigma \cdot \tau) \equiv \varnothing$ if there is no such simplex. Thus

$$
\begin{aligned}
N(\bar{L}, \bar{J}) & =\bigcup\{\bar{A} \bar{D}(\sigma, p) \mid(A, \sigma) \in S\}, \\
\dot{N}(\bar{L}, \bar{J}) & =\bigcup\{\bar{D}(\sigma, p) \mid \exists A \ni(A, \sigma) \in S\}, \\
N(\bar{L}, \bar{J}) \cap \bar{J}_{q} & =\dot{N}(\bar{L}, \bar{J}) \cup N(\bar{L}, \bar{K}) .
\end{aligned}
$$

Define $g: \bar{J} \rightarrow J^{\prime}$ by the conditions that

$$
g\left|\bar{J}_{q}=g_{q}, \quad g\right| \bar{A} \bar{D}(\sigma, p)=\left(g_{q} \mid \bar{A}\right) *\left(g_{q} \mid \bar{D}(\sigma, p)\right) \quad \text { if }(A, \sigma) \in S .
$$

Then $g$ is a well-defined homeomorphism and $(\bar{J}, \bar{K}, \bar{L} ; g)$ is a triangulation of $\left(\left|J^{\prime}\right|,\left|K^{\prime}\right|,\left|L^{\prime}\right|\right)$.

Clearly $\bar{L} \triangleleft \bar{K} \triangleleft \bar{J}$. Also $\bar{J}=N(\bar{K}-\bar{L}, \bar{J})$ because every principal simplex of $\bar{J}$ is of the form $\bar{A} B$ where $\bar{A}<\bar{L}$ ( $\bar{A}$ may be empty) and $B$ is a principal simplex of $\bar{D}(\sigma, p)$ for some $\sigma<K-L$. Thus by (iii), $B \cap(\bar{K}-\bar{L}) \neq \varnothing$.

Finally $\dot{N}(\bar{K}-\bar{L}, \bar{J})-\bar{L}$ is locally collared in $\bar{J}$. For, we claim that $g(\dot{N}(\bar{K}-\bar{L}, \bar{J})-\bar{L})=C\left(K^{\prime}-L^{\prime}, \partial J^{\prime}\right)-L^{\prime}$. The latter complex is locally collared in the manifold $J^{\prime}-\left(K^{\prime} \cup L^{\prime}\right)$ since, by $(5.4)$ it is an $(n-1)$-dimensional submanifold of $\partial\left(J^{\prime}-\left(K^{\prime} \cup L^{\prime}\right)\right)$. To verify the claim, suppose that $F$ is a simplex of $\dot{N}(\bar{K}-\bar{L}, \bar{J})-\bar{L}$. Then $F=\bar{A} B$ where $\bar{A}<\bar{L}$ (possibly $\bar{A}$ is empty) and $B<\bar{D}(\sigma, p)$ for some $\sigma<K-L$. Actually $B<\bar{D}(\sigma, p) \cap \bar{C}$ since $F \cap(\bar{K}-\bar{L})=\varnothing$. But $g \mid \bar{C}$ is a simplicial isomorphism. Hence $g(B)$ is a simplex of $C\left(K^{\prime}, \partial J^{\prime}\right) \cap D(\sigma, p)$ and $g(F)=A * g(B)$ is a simplex of $J^{\prime}$. In fact $g(F)<\partial J^{\prime}$ because $A<\partial J^{\prime}, g(B)<\partial J^{\prime}$ and $\partial J^{\prime}$ is full in $J^{\prime}$. [Proof that $A<\partial J^{\prime}$ : Let $\tilde{L}=\mathrm{Lk}\left(A, J^{\prime}\right)$. Then $\varnothing \neq g(B)<\tilde{L} \cap$ $C\left(K^{\prime}, \partial J^{\prime}\right)$. Thus $p \mid \tilde{L} \neq 1$. But $p \mid \tilde{L}$ is a retraction and $\tilde{L} \searrow p \tilde{L}$. So $\tilde{L}$ is not a sphere.] Therefore $g(F)<\partial J^{\prime}, g(F) \nless L^{\prime}$ since $F \nless \bar{L}$, and $g(F) \cap\left(K^{\prime}-L^{\prime}\right)=g(F \cap(\bar{K}-\bar{L}))$ $=\varnothing$. This proves that $g(F)<C\left(K^{\prime}-L^{\prime}, \partial J^{\prime}\right)-L^{\prime}$. The opposite inclusion is proven in a similar manner. 
We apply the stellar neighborhood theorem to the triangulation $(\bar{J}, \bar{K}, \bar{L} ; h g)$ of $\left(V, X_{R}, Y_{R}\right)$. The proof of part (a) is complete.

Proof of (9.1b). We assume that $M-(X \cup Y)$ is a manifold without boundary. Hence, by invariance of domain and assumptions (2)-(4),

$$
\partial(V-X)=\operatorname{Bdy}_{M-(X \cup Y)}(V-X)=\operatorname{Bdy}_{M-X}(V-X)=\left(\operatorname{Bdy}_{M} V\right)-Y .
$$

Therefore $\left(\operatorname{Bdy}_{M} V\right)-Y$ is locally bicollared in $M$ and

$$
\partial(V-X) \cup Y_{R}=\left(\operatorname{Bdy}_{M} V\right) \cup Y_{R} .
$$

Let $W$ be a regular neighborhood of $X \bmod Y$ in $M$. Arguing as above,

$$
\partial(W-X) \cup Y_{R}=\left(\operatorname{Bdy}_{M} W\right) \cup Y_{R} .
$$

By (9.1a) there exists a homeomorphism $f: V \rightarrow W$ such that $f \mid X_{R}=1$. Clearly $f(\partial(V-X))=\partial(W-X)$. If $(J, K, L ; h)$ is a stellar triangulation of $\left(W, X_{R}, Y_{R}\right)$ in which $h \dot{N}(K-L, J)=\left(\operatorname{Bdy}_{M} W\right) \cup Y_{R}$ then $\left(J, K, L ; f^{-1} h\right)$ is an analogous triangulation of $\left(V, X_{R}, Y_{R}\right)$. The result now follows from the stellar neighborhood theorem (6.1b).

Proof of (9.1c). We now assume hypotheses (2)-(5) of (9.1) and also that $M$ is a manifold and that $V_{1} \equiv \mathrm{Cl}[(V \cap \partial M)-Y]$ is a regular neighborhood of

$$
(X \cap \partial M) \bmod (Y \cap \partial M)
$$

in $\partial M$. Let $W$ be a regular neighborhood of $X \bmod Y$ in $M$. We denote

$$
\begin{aligned}
X_{1} & =\mathrm{Cl}[(X \cap \partial M)-(Y \cap \partial M)]=(X \cap \partial M)_{R}, \\
Y_{1} & =X_{1} \cap(Y \cap \partial M)=(Y \cap \partial M)_{R}, \\
W_{1} & =\mathrm{Cl}[(W \cap \partial M)-Y] .
\end{aligned}
$$

By part (a) there is a homeomorphism $f: V \rightarrow W$ such that $f \mid X_{R}=1$. Clearly $f(\partial V)=\partial W$ (where, as before, $\partial V$ and $\partial W$ denote the intrinsic $(n-1)$-skeletons of $V$ and $W$ ). We shall modify $f$ to get a new homeomorphism which also takes $V_{1}$ onto $W_{1}$.

We claim that $V_{1}$ is a regular neighborhood of $X_{1} \bmod (Y \cap \partial V)$ in $\partial V$. For, since $V_{1}$ is a regular neighborhood of $(X \cap \partial M) \bmod (Y \cap \partial M)$ in $\partial M$, there is a stellar triangulation $(J, K, L ; h)$ of

$$
\left(V_{1}, X_{1}, Y_{1}\right)=\left(V_{1}, \mathrm{Cl}\left(X_{1}-(Y \cap \partial V)\right), \mathrm{Cl}\left(X_{1}-(Y \cap \partial V)\right) \cap(Y \cap \partial V)\right)
$$

such that $h \dot{N}(K-L, J)=\left(\operatorname{Bdy}_{\partial M} V_{1}\right) \cup Y_{1}=\left(\mathrm{Bdy}_{\partial V} V_{1}\right) \cup Y_{1}$. [The last equality follows from invariance of domain since it is equivalent to the assertion that

$$
\left(\operatorname{Int}_{\partial M} V_{1}\right)-Y_{1}=\left(\operatorname{Int}_{\partial V} V_{1}\right)-Y_{1}
$$

where $\partial M$ and $(\partial V)-Y$ are $(n-1)$-manifolds without boundary and $V_{1} \cap Y=Y_{1}$.] Thus the claim follows from the stellar neighborhood theorem $(6.1 \mathrm{~b})$ once we know that $\left(\operatorname{Bdy}_{\partial V} V_{1}\right)-(Y \cap \partial V)$ is locally bicollared in $\partial V$. This is true because 
the latter set is just $\mathrm{Bdy}_{(\partial V)-Y}\left(V_{1}-Y\right)$ where $V_{1}-Y$ is an $(n-1)$-manifold in the open $(n-1)$-manifold $(\partial V)-Y$.

The argument above also applies to $W_{1}$ (see (2.13)). Thus $f\left(V_{1}\right)$ and $W_{1}$ are both regular neighborhoods of $X_{1} \bmod (Y \cap \partial W)$ in $\partial W$. But $\left(X_{R} \cap \partial W\right)=$ $X_{1} \cup(Y \cap \partial W)$ and $\left(W-X_{R}\right)$ is an $n$-manifold with $\left(\partial W-X_{R}\right)$ as an open subset of its boundary. Hence, by (7.8) there is a homeomorphism $G: W \rightarrow W$ such that $G \mid X_{R}=1$ and $G f\left(V_{1}\right)=W_{1}$. Let $h=G f$.

Since $h\left(V_{1}\right)=W_{1}$ it follows that $h(V, V \cap \partial M)=(W, W \cap \partial M)$. Thus, by invariance of domain,

$$
h\left(\left(\operatorname{Bdy}_{M} V\right) \cup Y_{R}\right)=\left(\operatorname{Bdy}_{M} W\right) \cup Y_{R}
$$

Let $\left(J, K, L ; h_{0}\right)$ be a stellar triangulation of $\left(W, X_{R}, Y_{R}\right)$ such that $h_{0} \dot{N}(K-L, J)$ $=\left(\operatorname{Bdy}_{M} W\right) \cup Y_{R}$. Then $\left(J, K, L ; h^{-1} h_{0}\right)$ is such a triangulation of $\left(V, X_{R}, Y_{R}\right)$. Since $\left(\operatorname{Bdy}_{M} V\right)-Y=\operatorname{Bdy}_{M-Y}(V-Y)$, and since $(V-Y)$ is an $n$-dimensional submanifold of $(M-Y)$ meeting $\partial(M-Y)$ in an $(n-1)$-dimensional submanifold, we know that $\left(\operatorname{Bdy}_{M} V\right)-Y$ is locally bicollared in $M$. Hence, by the stellar neighborhood theorem, $V$ is a regular neighborhood of $X \bmod Y$ in $M$, q.e.d.

\section{REFERENCES}

[A] M. A. Armstrong, Extending triangulations, Proc. Amer. Math. Soc. 18 (1967), 701-704.

[C] M. M. Cohen, Simplicial structures and transverse cellularity, Ann. of Math. 85 (1967), 218-245.

[H-Z] J. F. P. Hudson and E. C. Zeeman, On regular neighborhoods, Proc. London Math. Soc. (3) 14 (1964), 719-745.

- Corrections to "On regular neighborhoods", (to appear).

[H] L. Husch, On relative regular neighborhoods, Proc. London Math. Soc. (to appear).

[L] W. B. R. Lickorish, The piecewise linear unknotting of cones, Topology 4 (1965), 67-91.

[S] A. Scott, Infinite regular neighborhoods, J. London Math. Soc. 42 (1967), 245-253.

[T] R. Tindell, A counterexample on relative regular neighborhoods, Bull. Amer. Math. Soc. 72 (1966), 892-893.

[W] J. H. C. Whitehead, Simplicial spaces, nuclei and m-groups, Proc. London Math. Soc. 45 (1939), 243-327.

[Z] E. C. Zeeman, Seminar on combinatorial topology, Institut des Hautes Etudes Scientifiques, Paris, 1963 (mimeographed).

Princeton University,

Princeton, New Jersey 